Clemson University

TigerPrints

May 2020

\title{
Design of a Single Layer Metamaterial for Pressure Offloading in Transtibial Amputees
}

Nathan Kenneth Brown

Clemson University, nkbrown@g.clemson.edu

Follow this and additional works at: https://tigerprints.clemson.edu/all_theses

\section{Recommended Citation}

Brown, Nathan Kenneth, "Design of a Single Layer Metamaterial for Pressure Offloading in Transtibial Amputees" (2020). All Theses. 3292.

https://tigerprints.clemson.edu/all_theses/3292

This Thesis is brought to you for free and open access by the Theses at TigerPrints. It has been accepted for inclusion in All Theses by an authorized administrator of TigerPrints. For more information, please contact kokeefe@clemson.edu. 


\section{DESIGN OF A SINGLE LAYER METAMATERIAL FOR PRESSURE OFFLOADING IN TRANSTIBIAL AMPUTEES}

A Thesis
Presented to
the Graduate School of
Clemson University
In Partial Fulfillment
of the Requirements for the Degree
Master of Science
Mechanical Engineering

by

Nathan Brown

May 2020

Accepted by:

Dr. Georges Fadel, Committee Chair

Dr. John Desjardins

Dr. Gang Li

Distinguished External Reader: Dr. Anthony Garland, Sandia National Laboratories 


\begin{abstract}
While using a prosthesis, transtibial amputees can experience pain and discomfort brought on by significant changes in pressure across a finite area of skin, known as pressure gradients, at the interface between the residual limb and prosthetic socket. These pressure gradients can lead to dermatological issues, deep tissue damage, and prolonged joint and muscle pain. Current prosthetic interface solutions attempt to alleviate these pressure gradients by using highly compliant homogenous liners to distribute and therefore reduce pressures. This research investigates an approach to reduce peak pressure gradients around the limb through the design of a new inlay made from artificially structured materials, termed metamaterials, with tailored mechanical properties to act as an interface between the prosthetic socket and residual limb. The inlay is fabricated from a hyperelastic base material and has a triangular patterned unit cells which can be $3 D$ printed with walls of various slopes. By adjusting the unit cell wall slopes and thicknesses, the metamaterial hyperelastic material properties can be customized. The hyperelastic material properties of this metamaterial are modeled using a third order representation, namely a Yeoh $3^{\text {rd }}$ Order Hyperelastic Model. The $3^{\text {rd }}$ Order Coefficients from this model can be adjusted and optimized, then these optimal hyperelastic material property parameters can be mapped back into the physical design space as changes in the unit cell wall thickness or slope to create an inlay that can meet the unique offloading needs of an amputee. The layout of this metamaterial within the inlay can also be adjusted and optimized to better adapt to the unique limb shape of an amputee. Furthermore, the material properties and layout of the metamaterial can be optimized simultaneously to design a customizable inlay solution that can even better meet the unique performance needs of an amputee. Multiple finite element analyses simulations evaluate the pressure gradient reduction capabilities of the metamaterial inlay. A series of inlays were designed through the optimization of metamaterial properties and layout and compared to a prosthetists' prescription for the same patients. The metamaterial inlay shows, in all cases implemented, a greater reduction in peak pressure gradients than that of a common homogeneous silicone liner. The results show the potential feasibility of implementing this metamaterial as a customizable interface solution to meet the unique performance needs of individual transtibial amputees to better increase comfort and functionality.
\end{abstract}




\section{DEDICATION}

To my parents, for their endless love and support in all that I do. 


\section{ACKNOWLEDGMENTS}

I cannot begin to thank Dr. Georges Fadel and Dr. Anthony Garland enough for their time, effort, and support during these past two years. Without the constant encouragement, enthusiasm, patience, and immense knowledge from these two I would not have been able to develop this research to the point it is today. Dr. Fadel and Dr. Garland helped develop my love for research and for the desire to continue to push my research to higher levels. I will forever be thankful for the opportunity to work with these two incredible researchers and I look forward to continuing this relationship as I move forward with my PhD.

I would also like to show my deepest gratitude to Dr. John D. Desjardin and Meredith Owen from Clemson's Bioengineering department. Their vision of the full potential of this project allowed for me to be a part of an incredible research team. I was incredibly lucky to be able to work with such great minds who strive to better the lives of so many worthy people through their research. Their determination has shown me how much good can be done for the world when research targets a worthy cause.

My sincere thanks also goes to Brian Kaluf from Ability Prosthetics and Orthotics for his clinical insight and expertise.

Finally, I would like to thank the countless number of people who took the time to look over my work and offer their insight, especially Dr. Gang Li, Steven Iannucci, and Bailey Basso from Clemson's Mechanical engineering department, and of course my loving family. 


\section{TABLE OF CONTENTS}

\section{Page}

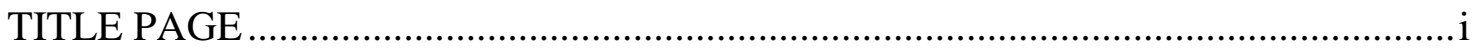

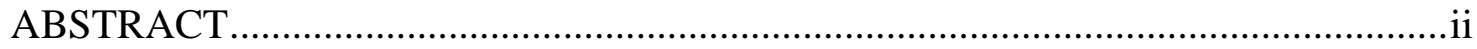

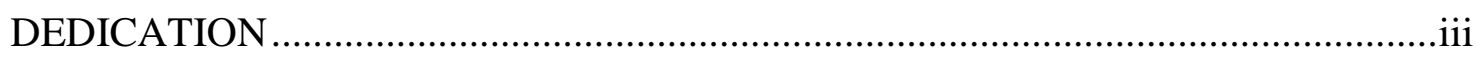

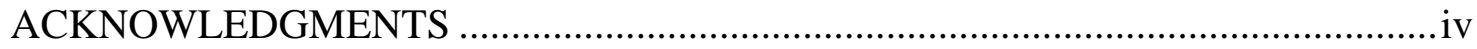

LIST OF TABLES .................................................................................... vii

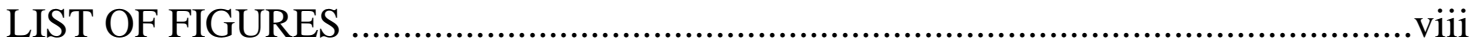

\section{CHAPTER}

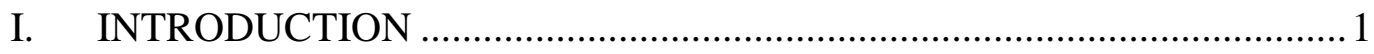

Residual Limb Comfort ..................................................................... 2

Interface Liners and Materials ........................................................ 5

Mechanical Metamaterials ............................................................. 12

Research Objectives ................................................................ 16

II. MECHANICAL METAMATERIAL OF INTEREST .............................20

Metamaterial Design .............................................................. 20

Material Properties .................................................................... 23

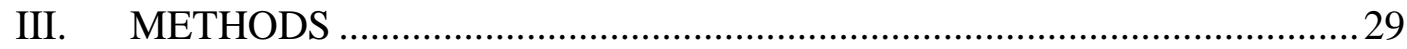

FEA Set Up ............................................................................ 29

Optimization Methods and Results ................................................. 39

Material Property Optimization ............................................... 40

Material Layout Optimization............................................. 43

Material Property and Layout Optimization ............................ 47

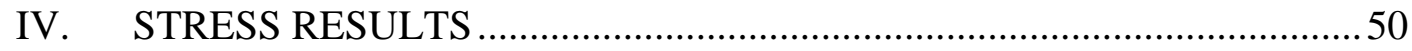

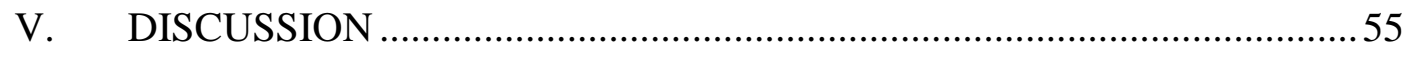

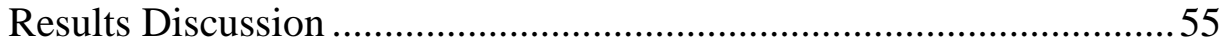




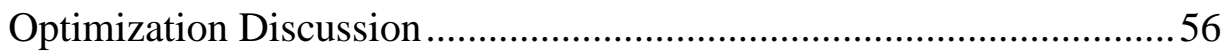

Material Property Optimization ....................................................56

Material Layout Optimization....................................................... 61

Material Property and Layout Optimization ..................................65

Clinical Relevance Discussion............................................................. 71

VI. FUTURE WORK AND CONCLUSION …………………..............74

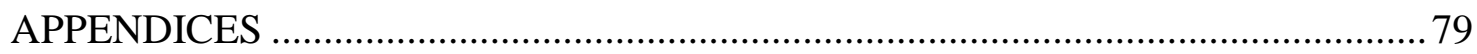

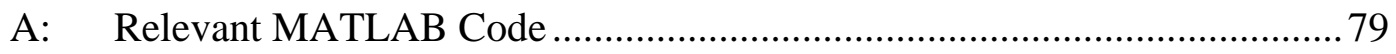

B: Limb Surface Stress Mappings ………………….....................................79

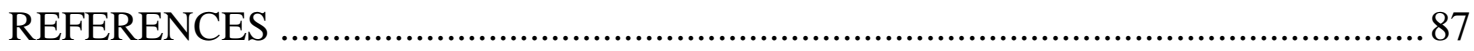




\section{LIST OF TABLES}

Table

Page

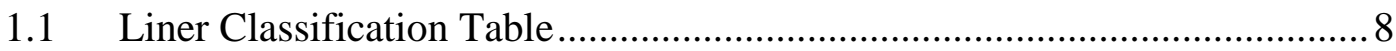

2.1 Shore O Hardness Values for Each Metamaterial Variation .......................22

2.2 Yeoh $3^{\text {rd }}$ Order Material Coefficients of Each Metamaterial and Base Composite Material ..........................................................26

2.3 $\quad \mathrm{R}^{2}$ Values for Material Coefficients with Respect to Compression Testing Results.........................................................27

3.1 Optimized Yeoh $3^{\text {rd }}$ Order Material Coefficients at the Fibular Head.

3.2 Optimized Yeoh $3^{\text {rd }}$ Order Material Coefficients at the

Tibial End and Patellar Tendon

3.3 Optimized Material Layout at the Fibular Head .....................................46

3.4 Optimized Material Layout at the Patellar Tendon and

Tibial End.

3.5 Optimized Material Coefficients and Layout at the

Fibular Head.

3.6 Optimized Material Coefficients and Layout at the

Patellar Tendon and Tibial End

4.1 Peak Stress and Peak Pressure Gradient Comparison at

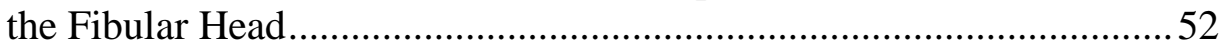

4.2 Peak Stress and Peak Pressure Gradient Comparison at

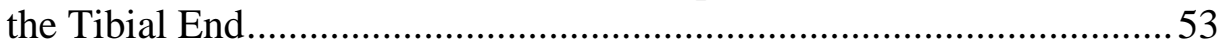

4.3 Peak Stress and Peak Pressure Gradient Comparison at the Patellar Tendon 


\section{LIST OF FIGURES}

Figure

1.1 Anatomic Representation of Right Residual Limb ................................ 2

2.1 Metamaterial Physical Design ...........................................................21

2.2 Wall Thickness Variances of Each Draft Angle ...................................23

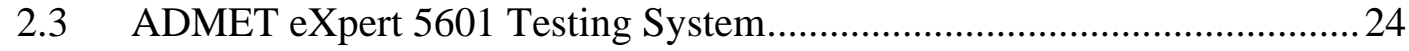

2.4 Stress-Strain Results of Experimental Compression Testing .....................25

2.5 Stress-Strain Comparison Between Experimental

Compression Results and Yeoh $3^{\text {rd }}$ Order Material Coefficients ........................................................................... 27

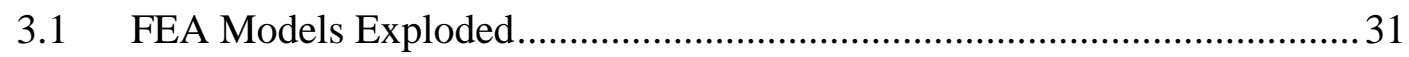

3.2 Isolated FH Exploded View for Limb Shape 1 ...................................... 32

3.3 Limb Shape 1 Prosthetist's Prescribed Inlay ........................................ 33

3.4 Limb Shape 2 Prosthetist's Prescribed Inlay ....................................... 35

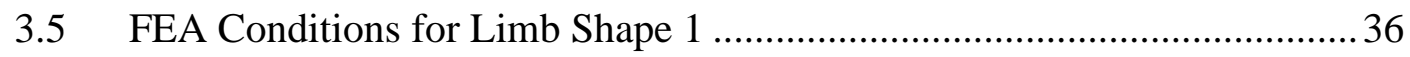

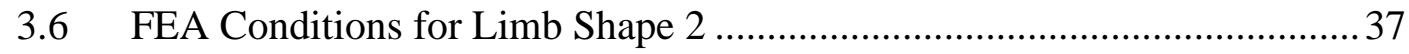

3.7 Mesh Convergence Study Results ................................................... 38

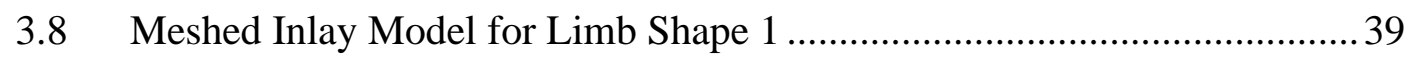

3.9 Geometric Variables on Inlay at the Fibular Head ..................................43

3.10 Geometric Variables on Inlay at the Patellar Tendon

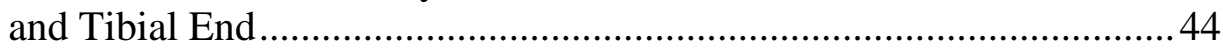

4.1 Limb Surface Stress for Limb Shape 1 at the Fibular Head .......................50

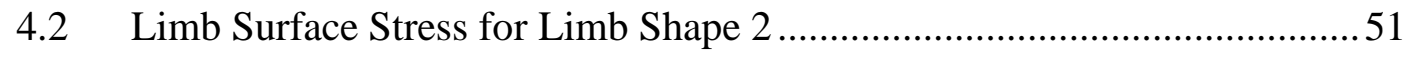


List of Figures (Continued)

Figure

Page

5.1 Stress-Strain Comparison Between "Optimized Material

Properties" and Current Material Properties at the

Fibular Head.

5.2 Stress-Strain Comparison Between "Optimized Material

Properties" and Current Material Properties at the

Tibial End and Patellar Tendon

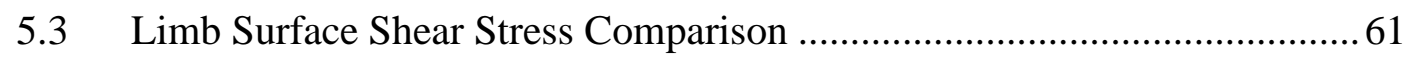

5.4 Fibular Head Inlay Material Layout Dimensions .........................................62

5.5 Patellar Tendon Inlay Material Layout Dimensions ......................................63

5.6 Tibial End Inlay Material Layout Dimensions ...............................................64

5.7 Fibular Head Inlay Material Layout Dimensions

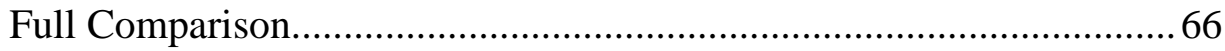

5.8 Stress-Strain Comparison for Fibular Head Inlay

Material Property Optimization with Material

Layout Optimization

5.9 Patellar Tendon Inlay Material Layout Dimensions

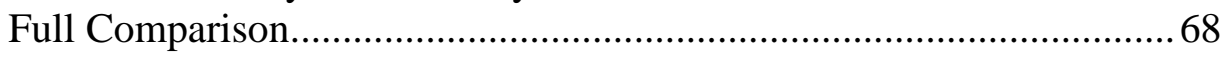

5.10 Stress-Strain Comparison for Patellar Tendon Inlay

Material Property Optimization with Material

Layout Optimization

5.11 Tibial End Inlay Material Layout Dimensions

Full Comparison.

5.12 Stress-Strain Comparison for Tibial End Inlay

Material Property Optimization with Material

Layout Optimization 70 


\section{List of Figures (Continued)}

Figure

B.1 Limb Surface Stress [MPa] with Silicone Liner at the Fibular Head

B.2 Limb Surface Stress [MPa] with Practitioner Prescribed Inlay at the Fibular Head.

B.3 Limb Surface Stress [MPa] with Optimized Material

Properties Inlay at the Fibular Head

B.4 Limb Surface Stress [MPa] with Optimized Material

Layout Inlay at the Fibular Head

B.5 Limb Surface Stress [MPa] with Optimized Material

Properties and Layout Inlay at the Fibular Head

B.6 Limb Surface Stress [MPa] with Silicone Liner at the Tibial End

B.7 Limb Surface Stress [MPa] with Practitioner Prescribed Inlay at the Tibial End.

B.8 Limb Surface Stress [MPa] with Optimized Material Properties Inlay at the Tibial End

B.9 Limb Surface Stress [MPa] with Optimized Material Layout Inlay at the Tibial End

B.10 Limb Surface Stress [MPa] with Optimized Material Properties and Layout Inlay at the Tibial End

B.11 Limb Surface Stress [MPa] with Silicone Liner at the Patellar Tendon

B.12 Limb Surface Stress [MPa] with Practitioner Prescribed Inlay at the Patellar Tendon

B.13 Limb Surface Stress [MPa] with Optimized Material Properties Inlay at the Patellar Tendon 


\section{List of Figures (Continued)}

Figure

Page

B.14 Limb Surface Stress [MPa] with Optimized Material

Layout Inlay at the Patellar Tendon......................................................... 86

B.15 Limb Surface Stress [MPa] with Optimized Material

Properties and Layout Inlay at the Patellar Tendon...................................86 


\section{CHAPTER ONE}

\section{INTRODUCTION}

A lower limb prosthesis provides increased ambulatory function for an amputee. The amputee should feel confident that the prosthetic provides the comfort and functionality needed to complete routine tasks [1]. Given that $84 \%$ of amputees wear the prosthesis an average of 12 hours a day, comfort is vital [2]. Greater prosthetic use has been associated with higher levels of independence, improved perceived quality of life, and greater employment success. This improved lifestyle has prolonged benefits including greater self-confidence and self-image [1]. While some amputees find a prosthesis to be a permanent and effective replacement to the absent limb, satisfaction with current systems remains alarmingly low due to persistent issues of comfort and fit.

A prosthesis usually subjects the residual limb to unnatural loading conditions compared to the residual limb of a non-amputee, leading to discomfort, dermatological issues, deep tissue damage, and prolonged joint and muscle pain [3-7]. Understanding the pressure distributions around the limb is often the best way to properly counteract the discomfort experienced by lower-limb amputees [8]. Most studies show the largest stress concentrations around the Patellar Tendon (PT), Tibial Crest (TC), Fibular Head (FH) and/or the Tibial End (TE) as referenced in Figure 1.1 [9-12]. 


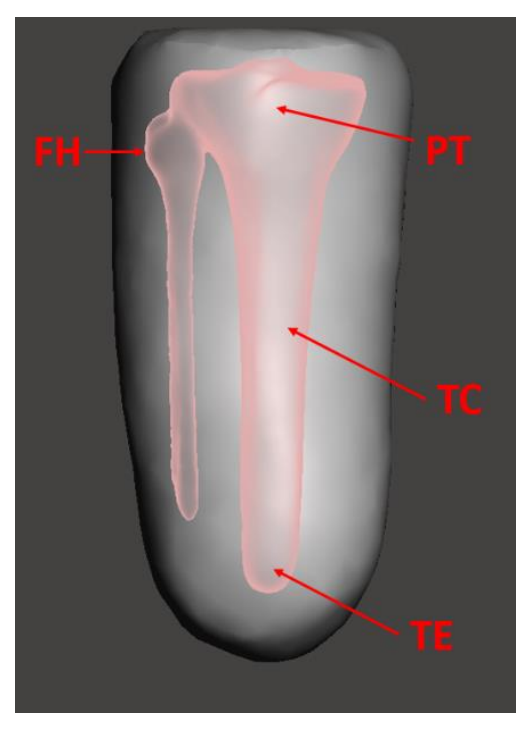

\section{Figure 1.1. Anatomic Representation of Right Residual Limb (Patellar Tendon (PT), Fibular Head (FH), Tibial Crest (TC), and Tibial End (TE))}

The magnitudes of the peak stress (PS) are often the focus of studies, as this pressure is a key contributor to skin breakdown that can lead to many undesirable issues experienced by lower limb amputees. However, a determined range of pressure magnitudes that can predict this skin breakdown does not exist [13-14]. Peak pressure gradient (PPG), instead of PS, may play a larger role in predicting residual limb issues. PPG is the significant change in pressure across a finite area of skin at the interface between the residual limb and prosthetic socket. Large pressure gradients can contribute to skin breakdown because of the resultant large shearing stresses placed on the soft tissues [15], which emphasizes the need to decrease the PPG as well as the PS on an amputee's residual limb.

\subsection{Residual Limb Comfort}

It is important to understand the stress distributions around the residual limb to help guide the prescription of a prosthesis to achieve a comfortable experience for the user [8]. A lack of comfort while wearing the prosthesis is the most common complaint of lower 
limb amputees. Comfort, like pain, is very subjective for each patient, so it is important to implement the proper measurement techniques to better understand a patient's interactions with the prosthesis. Various descriptive terms and numerical scales have been used to describe pain. A common method is to ask patients to rate their Socket Comfort Score (SCS). Studies have shown that the SCS has consistently demonstrated validity as a socket comfort measure. Patients are asked to rate the comfort of their socket on a $0-10$ scale where 0 and 10 represent peak discomfort and peak comfort, respectively. The SCS is paired with a prosthetist's visual diagnosis of how well the socket appears to be fitting to the patient's limb. The prosthetist's diagnosis is driven by visual evidence of discomfort such as redness, pressure marks, or sores. A strong correlation exists between a low SCS score and the prosthetist's ability to spot visual evidence of a poor socket fit. Clear signs of patient discomfort will lead to socket and/or liner adjustments. These adjustments can involve a physical change of the socket shape or alteration in the material or offloading areas of the liner. These adjustments generally lead to an increase in the SCS. [16-18].

The SCS and other pain measuring systems are often ambiguous and rely on description terms to describe pain and/or comfort. They do not provide a quantifiable assessment of a patient's comfort [15]. Lee et al. [19] looked to quantify pain vs. comfort on the limb by investigating the regional load-bearing ability of transtibial residual limbs to determine what pressure thresholds would cause discomfort for a patient. The investigators gradually increased pressure individually around 11 key limb locations until the patient first noted the sensation of pain. The pressure was increased until the patient stated the pain was unbearable, which concluded the test. The pressure associated with the first recognition of 
pain was referred to as the pain threshold. The pressure value that prompted the patient to stop the experiment was recorded as the pain tolerance. Any pressure below a patient's pain threshold was considered a "comfortable" amount of pressure. Two trials were run for each of the 12 patients. The first trial used an indenter made of soft pelite and the second used an indenter made of polypropylene. The polypropylene was used to mimic the hardouter shell of a prosthetic socket, while the pelite represented a softer internal liner material.

Along with the pain tolerance and threshold results, [19] utilized a finite element analysis (FEA) to model the pressure distributions around the limb. The FEA results produced a pressure gradient that could be used to determine PPG at the critical limb areas for each patient. The pressure gradients varied for each patient based on the pain threshold readings from the original test. Unfortunately, the pain tolerance and threshold were highly variable. The results were not reliable enough to determine a definitive pressure gradient value that can be used as a target value to ensure comfort for an amputee.

Phantom sensations are the feeling of still having the missing extremity present. This feeling has been described as a "persistent, bothersome sensation". Phantom pain is the feeling of pain in the amputated part of the extremity. Phantom sensations and phantom pain are discomfort areas that can be very difficult to counteract. A study of 200 amputees found that approximately $54 \%$ complained of phantom sensation, while $17 \%$ complained of phantom pain [20]. Phantom pain is commonly classified as neuropathic and is assumed to be related to damage of central or peripheral neurons. Lesions of the peripheral nerves can also cause this sensation to arise. Phantom pain has also been tied to psychological factors. Grief of losing a limb can manifest into the painful sensation a patient experiences. 
Muscle relaxants, anticonvulsants, barbiturates, and neuroleptics are commonly prescribed to treat phantom pain and sensations. Only $30 \%$ of those treated for phantom pain experience noticeable benefit after seeking medical attention. Therefore, targeting pressure offloading on the residual limb may not result in a reduction in phantom pain and/or sensations. Unfortunately, the correlation between residual-limb and phantom-limb pain is unknown and little work has been done to prove that reducing residual limb pain leads to a reduction in phantom limb pain [21].

Hygiene problems remain a persistent challenge for transtibial amputees. The tight vacuum seal of traditional prosthetic liners can result in excessive heat and moisture within the socket. The liner acts as a heat insulator and entraps the thermal energy that is attempting to leave the residual limb, leading to elevated temperatures within the socket. The area between the liner and skin is known to be a place for the development of hygienic skin problems including contact dermatitis, hyperhidrosis, and bacterial infection, which can all lead to itching, rashes, and odor [22-24]. Nearly 60-70\% of lower limb amputees experience excess perspiration and odor from liners [6]. If these skin problems are not promptly addressed, they can lead to skin ulceration and blistering, which traditionally forces discontinued use of the prosthetic altogether [7]. Keeping the residual limb, socket, and liner clean has been shown to reduce direct skin problems, but hygiene problems remain to be resolved [6].

\subsection{Interface Liners and Materials}

Prosthetic liners are often implemented as an interface between the rigid socket and residual limb to transmit and distribute loads and reduce interface stresses [11]. The main 
purpose of these prosthetic liners is to alleviate the transfer of loads from the prosthetic socket to the residual limb. Prosthetists rely on personal intuition and experience when choosing the appropriate liner for a respective patient [5].

The first prosthetic liners were made from open and closed cell foams that were designed to wrap around the residual limb [25-26]. Recently, silicone and elastomer liners replaced foams as the most commonly implemented offloading solution because of increased durability and stress distributing capabilities compared to foams [5, 27]. Advancement of prosthetic liner materials has been a common focus of research [5].

Sanders and Daly et al. [25] altered the mechanical properties of foam liners through the utilization of vacuum-forming manufacturing of foam liners. The vacuum forming weakens the cell structure. By controlling the degree in which these cell structures are weakened, Sanders proposed that the foam liners could be altered to fit the performance needs of specific patients [16].

Sanders et al. [26] investigated the compressive stiffness and coefficient of friction (COF) of eight common liner materials. These materials were Spenco ${ }^{\circledR}$, Poron ${ }^{\circledR}$, silicone, soft Pelite, medium Pelite, firm Plastazote, regular Plastazote, and Nickelplast. These materials offered a range of stiffnesses as well as variability between materials with linear and non-linear elasticity. The compression testing mimicked conditions the liner would experience within the prosthetic socket. The coefficients of friction were evaluated against both skin and sock. The results suggested that there may be advantages to utilizing materials that acted similarly to biological tissues. Soft Pelite, medium Pelite, regular Plastazote, and firm Plastazote all showed non-linear behavior by increasing stiffness as 
displacement was increased, much like the response of soft tissue. Nickelplast, the stiffest material of the group, was deemed too stiff to be able to supply the appropriate cushioning needed for the residual limb. With regards to COF, Sanders determined that the materials with the lowest COF (Spenco and Poron) could be problematic in maintaining proper suspension. Without proper suspension, there would be drastic slipping between the limb and liner, which would deem the entire prosthetic solution a failure.

Emrich and Slate et al. [28] attempted to better match in vivo loading conditions by testing a series of material properties under cyclic compressive loading, shear loading, and frictional loading. The materials tested were Bock-Lite, Pedilin, silicone, and polyurethane. Bock-lite and silicone lasted significantly longer under compressive loading than Pedilin and polyurethane before failure. Unfortunately, silicone and polyurethane were not able to be tested under abrasive loading because the materials were prone to tear. However, Bocklite was able to survive 15 times as many cycles as the pedilin when tested under abrasive loading. Polyurethane and silicone showed the highest COF from the final portion of the study. Unfortunately, the researchers could not recommend a liner material that was superior in all categories.

To further the liner material research, Sanders et al. [29] expanded to a 15-liner material study. The researchers tested these materials under compression, friction, and shear. The materials were evaluated based on "Compressive Stiffness", "Coefficient of Friction", "Shear Stiffness", and "Tensile Stiffness". The liners were rated on a scale from 1-4. A summary of the comparative results can be seen in Table 1.1 with 1 and 4 correlating to the group of materials with the smallest and largest values, respectively. 
Table 1.1 Liner Classification Table (1=Smallest Values, 4=Largest Values) [29]

\begin{tabular}{|c|c|c|c|c|}
\hline Liner & $\begin{array}{c}\text { Compressive } \\
\text { Stiffness } \\
\end{array}$ & $\begin{array}{l}\text { Coefficient of } \\
\text { Friction } \\
\end{array}$ & $\begin{array}{c}\text { Shear } \\
\text { Stiffness } \\
\end{array}$ & $\begin{array}{l}\text { Tensile } \\
\text { Stiffness }\end{array}$ \\
\hline \multicolumn{5}{|l|}{ Gels } \\
\hline ELDT $32(3,6)$ & 1 & 3 & 1 & 1 \\
\hline Super Stretch & 1 & 3 & 1 & 1 \\
\hline Alpha Liner & 1 & 1 & 1 & 1 \\
\hline SiloLiner & 1 & 1 & 1 & 1 \\
\hline \multicolumn{5}{|l|}{ Elastomers } \\
\hline DERMO Liner $(6,9)$ & 2 & 1 & 2 & 1 \\
\hline Iceross Comfort & 2 & 1 & 2 & 1 \\
\hline Iceross Clear & 4 & 2 & 3 & 3 \\
\hline Iceross Two Color & 4 & 3 & 3 & 2 \\
\hline Clearpro & 4 & 2 & 3 & 2 \\
\hline Fillauer Silicone & 4 & 2 & 4 & 4 \\
\hline AEGIS & 4 & 2 & 4 & 3 \\
\hline AEGIS Z & 3 & 3 & 2 & 1 \\
\hline \multicolumn{5}{|l|}{ Urethane } \\
\hline TEC Pro 18 & 3 & 4 & 3 & 1 \\
\hline
\end{tabular}

The authors recommended that, under compression, the softer silicone gels would be better suited to cushion the boney prominences of the residual limb, while the stiffer silicone elastomer and urethane would be advantageous for patients with a significant amount of soft tissue. All the tested samples had large enough COF's to limit slipping. The shear stiffness results were similar to those of the compression testing. 
Another research team [12] focused on the thickness of gel liners as compared to the liner material itself. The study investigated the effects of gel liner thickness on peak socket pressures around the fibular head. Most of the subjects perceived increased comfort with a thicker liner. The increased comfort was linked with reduced fibular head stresses. The thicker liners were assumed to be more comfortable because of increased compliance. The authors proposed a thicker gel liner would reduce pressures around boney prominences as compared to thinner liners. In future research, the authors are attempting to determine optimal gel thicknesses for individual patients to provide a more customized solution to match the needs of the individual. The research presented in this thesis found that patients found thicker liners to feel "squishy" over time. The patients did not have confidence that the thicker liner would be able to provide a long-term liner solution, which contrasts the results found by [12].

As stated above, hygiene is a big concern for amputees. A lack of proper hygiene can wreak havoc on an amputee's lives. Excessive heat and moisture retention are driving factors for the discomfort complaints of lower limb amputees [30-34]. Therefore, heat and moisture transfer properties of liner materials have been another topic of research.

Hachisuka [35] investigated the moisture permeability properties of different liner and socket materials. The liners tested included a silicone gel liner, Icelandic Roll-On Silicone Socket (ICEROSS) liner, and pelite. The permeability was tested for the prosthetic sockets alone, the liners alone, and a liner inside the socket. The liners placed into the sockets were more than 80 times less permeable than the sockets alone. This suggests that liners are 
highly impermeable to moisture transfer, validating the claim that prosthetic liners are ideal areas for moisture development.

Klute et al. [36] focused on the heat transfer properties by investigating the thermal conductivity of different prosthetic socket and liner materials. Twenty-tree different liners were tested with a thermal conductivity coefficient range of $0.085-0.266 \mathrm{~W} / \mathrm{m}-\mathrm{K}$. The conclusion of this study emphasized that liners are highly resistive to heat conduction and therefore play a major role in the elevated skin temperatures found around the residual limb. These two studies suggest that the liner should only be targeted around areas that require pressure offloading in order to limit the amount of heat and moisture capture around the limb.

Klute et al. [5] expressed concern that there is little scientific evidence to guide a prosthetist in the liner prescription process. The research shows that liners can help distribute pressures and can lead to increased comfort. However, there is limited evidence in the difference between the potential benefits of each material for specific patients.

Hafner et al. [37] looked to further understand the prosthetists' liner selection practices. The results of this survey driven study showed that the liner manufacturers were the primary source for information on available liner products. Liner characteristics, like durability, comfort and suspension, are often the driving factors in the selection of a liner. Even though there are more than 70 available liner solutions on the market, the study's respondents reported only having prescribed 16 of the 70 liners to their patients. Of those 16 , the respondents said they routinely only used $2-3$ liners to meet the needs of their patients. These prescriptions most commonly utilized silicone, thermoplastic elastomer, 
and urethane. The study concluded by emphasizing the need for an objective tool or resource to better pair individual patients with liner solutions that meet the unique performance needs of each amputee.

Nearly all transtibial amputee research has been limited to the use of homogenous material liners. A significant gap is apparent in the need to investigate non-homogenous liners. The research suggests that boney prominences should be offloaded by softer materials while soft tissue benefits from the suspension capabilities of stiffer materials. A homogenous liner would not be able to satisfy this recommendation. A literature review for non-homogenous liners was launched and results were limited to two patents (US6702858B2) and (US6136039).

US6136039 describes a dual durometer silicone liner. The liner is comprised of a soft inner silicone elastomer layer coupled with a stiffer outer silicone elastomer layer. The softer inner layer acts to conform to the residual limb, while the stiffer outer layer provides the strength necessary to meet the physical demands of an amputee. The softer inner layer is thicker towards the distal end of the liner and gets thinner moving closer to the knee. This increase in thickness is meant to supply additional cushioning to the distal end of the limb. This is similar to the proposal made by Boutwell et al [12] that boney prominences require additional cushioning. The liner is fabricated in a number of different sizes to accommodate to a variety of residual limb shapes and sizes. Unfortunately, this liner solution does not provide the possibility of customization to meet the exact needs of a given patient. 
US6702858B2 describes a liner that uses variable viscosity fluid. The variable viscosity fluid can alter the stiffness of the liner. The liner is designed to accommodate the swelling and/or contracting of the residual limb. The viscosity of the fluid varies in response to the loading on the liner. The goal of the liner is to increase the viscosity during increased loading for a stiffer liner and the opposite during times of decreased loading. This liner aims to increase the stiffness around areas of high stress and decrease the stiffness around areas of lower stress. Boney prominences are traditionally the areas of high stress around the limb and the soft tissues are areas of lower stress. Hence, this liner aims to achieve the opposite of what has been previously proposed that softer materials should be targeted around boney prominences to provide additional cushioning.

Beyond these two patents, there is very limited research looking into implementing multi-stiffness liner solutions that target the unique needs of individual patients. The reasoning behind this lack of research is unknown. One reason may be a lack of knowledge in methods to develop such a liner. A potential solution to this problem could arise through the utilization of mechanical metamaterials.

\subsection{Mechanical Metamaterials}

Metamaterial research first originated in the field of optics, acoustics and mechanics [38-41]. Mechanical metamaterials are man-made materials with mechanical properties defined by their material and physical structure as compared to those defined by their material composition only [42]. Metamaterials are able to achieve unique material properties such as zero or negative Poisson's ratios, vanishing shear modulus, negative stiffness, negative compressibility, singularly nonlinear behavior, and customized 
topological microstructures [43-45]. Metamaterials can be classified into specific groups based on the elastic constant that is most notably affected by the introduction of the material and physical alteration. The groups are broken into emphasis on stiffness, shear/bulk moduli, or Poisson's ratio. Each of these groups are a relatively broad field of research. Therefore, based on the scope of the presented research, emphasis is placed on the metamaterial research that targets stiffness. The subsection of this classification of research involves micro-nanolattices, chiral/anti-chirals, origami metamaterials, cellular origami, and pattern transformation.

Although metamaterials are a broad class of fabricated material design research, the work presented in this thesis focuses on reviewing metamaterials with potential applications and relevance in prosthetic liners. Metamaterials pertaining to these applications generally have high compliance (softer than rubber), have a small form factor (approximately $3 \mathrm{~mm}$ unit size), are made from elastomers (have non-linear elasticity), and have adjustable design variables associated to the fabrication of the unit cell that directly alter the compressive response of the metamaterial.

Pattern transformation is a subset of metamaterials that undergoes a change in deformation mode at a specific load. The change in deformation mode occurs from a cooperative buckling of the unit cell. The magnitude of the deformation mode change, as well as the compressive force that causes the change in deformation mode, is dependent on the size, density, and orientation of the metamaterial unit cell. Pattern transformation is commonly seen in multi-layered metamaterials comprised of internal unit cells. Altering 
the size or shape of the pore can, for instance, lead to changes in the buckling reaction the material undertakes when subjected to compression [46].

Mullin et al. [47] looked at multi-layered periodic elastomeric cellular solids. The multi-layered metamaterial was subjected to uniaxial compression until a critical value was reached that caused the change in deformation mode of the stress-strain properties. A series of unique unit cell structures were creating using a pattern of circular, triangular, hexagonal, and pentagonal shapes, as well as a combination of more than one. The results of the study showed that the load at which the phase shift of the stress-strain occurred was dependent on the unit cell shape and size. Each metamaterial experienced a unique buckling reaction as the compressive force was increased. Some of the unit cells collapsed completely, while other did not. The study was conducted through both experiments and simulations, which showed excellent quantitative agreement. The researchers concluded that it is vital to select the appropriate unit cell shape to be customize the metamaterial's stress-strain properties, including the possibility of a change in deformation mode.

Overvelde et al. [48] investigated the effect pore shape plays on the buckling of multilayered metamaterials. Compression testing was run on three samples of silicone-based rubber, each with a different pore shape. One pore shape was circular, while the other two were novel "star-like" geometries. Each sample was comprised of an eight by eight set of these pores. The researchers were interested in observing the mechanical response of the metamaterials when buckling occurred. The shape of the pores not only affected the strain at which buckling occurred, but it affected the instability as well as the lateral contraction and compaction of the sample as a whole. The circular pore shape and one of the novel 
pore shapes were able to achieve complete lateral contraction under compression. The other novel pore shape lead to a non-uniform lateral deflection of the unit cell. Regardless of the compressive force that was applied to the material, the pores always returned to their initial shape. The results of the test show that the pore shape of a metamaterial unit cell can be altered to better achieve desired mechanical response.

The previously discussed research has shown that there are several ways to alter the physical properties of metamaterials to satisfy desired mechanical responses. However, the work was limited to multi-layered metamaterials. Multi-layered metamaterials utilize a series of unit cells acting together to deliver a desired mechanical response. Single-layer mechanical metamaterials are not as commonly investigated.

Yang et al. [49] investigated single-layer metamaterials. The research team looked at a metamaterial composed of a pantographic substructure that was periodic in space. The single-layer metamaterial had a small square patterned unit cell. Shearing and uniaxial tensile experiments were conducted on 3D printed models. The metamaterial showed hyperelastic material properties during the test which prompted a discussion about which hyperelastic material model should be used to describe the mechanical response of the system. The study investigated the St. Venant-Kirchhoff, Bidermna, Isihara, HaineWilson, Mooney-Rivlin 5 parameter, and Yeoh $3^{\text {rd }}$ order models. These material models were utilized in a series of numerical simulations to compare to the experimental results. The Yeoh $3^{\text {rd }}$ order model showed the best match with respect to the other models. The outcome of the study shows the need to have a proper method of numerically representing 
the mechanical response of these metamaterials, whether the elastic behavior is linear or non-linear.

Bodaghi et al. [50] investigated the large deformations of additively manufactured soft materials. These materials were made of soft poly-lactic acid and designed as a singlelayered patterned arrangement of unit-cells in parallelogram and hexagonal shapes. A set of experiments were conducted under in-plane tension and compression in axial and transverse directions. The results of these experiments showed that the unit-cell shape as well as the direction, type, and magnitude of the mechanical loading play a significant role on the mechanical response of the metamaterial. As with the research conducted by Yang et al. [49], the metamaterials in this research showed hyper-elastic behaviors and the researchers wanted to determine a numerical method to represent those behaviors. A series of finite element analysis were created using Mooney-Rivlin, non-linear Green-Lagranage, and Newton-Raphson methods to represent the mechanical response of the metamaterial. All three methods showed results matching the experimental results. The researchers determined that modeling of hyper-elasticity and large strain of the soft metamaterials is essential to the future of accurately representing additively manufactured single-layered metamaterials.

\subsection{Research Objectives}

Mechanical metamaterials are special materials that are capable of meeting a range of unique mechanical response needs. Development of an inlay for transtibial amputees that solves the problems of comfort and hygiene remains an important challenge. Metamaterials offer a potential solution by providing a solution with variable mechanical response that 
can be implemented to offload high stress areas of an amputee's residual limb. The metamaterial inlay can be designed to specifically target the areas of offloading without acting as a heat and moisture insulator, like common prosthetic liners.

Based on the recommendation from Sanders et al. [29], a method to implement an interface material between the residual limb and socket was sought, that could be soft at the boney prominences but stiffer around large concentrations of soft tissue. Members of the research team had previous experience working with a metamaterial with adjustable stiffness (Patent US10244818 B2) [58] that had been used in orthotics to offload pressure for patients with diabetic foot ulcers. While the original application of this metamaterial was orthotics, the designers of this metamaterial saw the potential of implementation at other anatomical locations such as the hip, head, knee, hand, and chin, which sparked the interested in using this metamaterial for this study. The desire to further investigate the potential of using this metamaterial as an interface material between the residual limb and prosthetic socket prompted the objectives of this research to be:

1. Determine the hyperelastic material properties of the selected metamaterial.

2. Determine a method to provide a simple representation of the material properties.

3. Determine the reduction in peak pressure gradient (PPG) on the residual limb with an inlay utilizing the metamaterial that has been designed by a prosthetist.

4. Optimize the design of the inlay to achieve a further reduction in PPG

To determine the peak pressure gradients, finite element analysis (FEA) was used on all residual limb simulations. Clinically testing the limb stress distributions can be challenging due to cost, time, and patient recruitment and retention [51]. Therefore, a significant 
amount of prosthetic research is completed with the aid of FEA. FEA has been deemed a valid alternative to in vivo testing to determine the stress, strains, and deformations of a residual limb [10-11, 52-57]. However, further research on human subjects would validate the current work. This is the subject of continuing research.

In order to accomplish the four research objectives, the following research plan was created:

1. Determine the mechanical response of the metamaterial through a series of uniaxial compression testing.

2. Represent the hyperelastic mechanical response of the metamaterial utilizing Yeoh $3^{\text {rd }}$ order coefficients.

3. Develop two unique transtibial amputee FEA models to be used to determine pressure offloading capabilities of the metamaterial inlay.

4. Using the FEA models, determine the PS and PPG on the residual limbs without a liner to provide a base line

5. Using the FEA models, determine the PS and PPG on the residual limbs utilizing a homogenous silicone liner to provide a common liner comparison

6. Using the FEA models, determine the PS and PPG on the residual limbs with an inlay utilizing the $3^{\text {rd }}$ order representation of the metamaterial as well as a material offloading layout prescribed by a prosthetist

7. Run a series of optimization algorithms to design inlays that can further reduce the PS and PPG on the residual. 
a. Optimize the Yeoh $3^{\text {rd }}$ order parameters but use the prosthetist's material layout prescription

b. Optimize the material layout but use the prosthetist's material representation prescription

c. Optimize both the Yeoh $3^{\text {rd }}$ order parameters and the material layout

8. Using the FEA models, determine the PS and PPG on the residual limbs with the three optimized inlay solutions

9. Compare the PPG reduction capabilities of all the inlay variations that utilize the metamaterial representation compared to the PPG results of the residual limb with no inlay as well as the residual limb with the silicone liner. 


\section{CHAPTER TWO \\ MECHANICAL METAMATERIAL OF INTEREST}

\subsection{Metamaterial Design}

The metamaterial, seen in FIGURE 2.1, is constructed by 3D printing walls of various tapered thicknesses controlled by draft angles. These various wall thicknesses leave a triangular patterned unit cell indented into the top surface of the base material. The unit cell pattern is comprised of four equal sized triangles spanning radially 180 degrees. The number and orientation of the individual triangles within the unit cell allow for the cell to be patterned in orthogonal directions without any overlapping of the individual triangles. Once patterned, the unit cells create a set of equilateral triangles on the top surface of the base material, and therefore individual unit become grouped together with other unit cells, as seen in Figure 2.1B. 

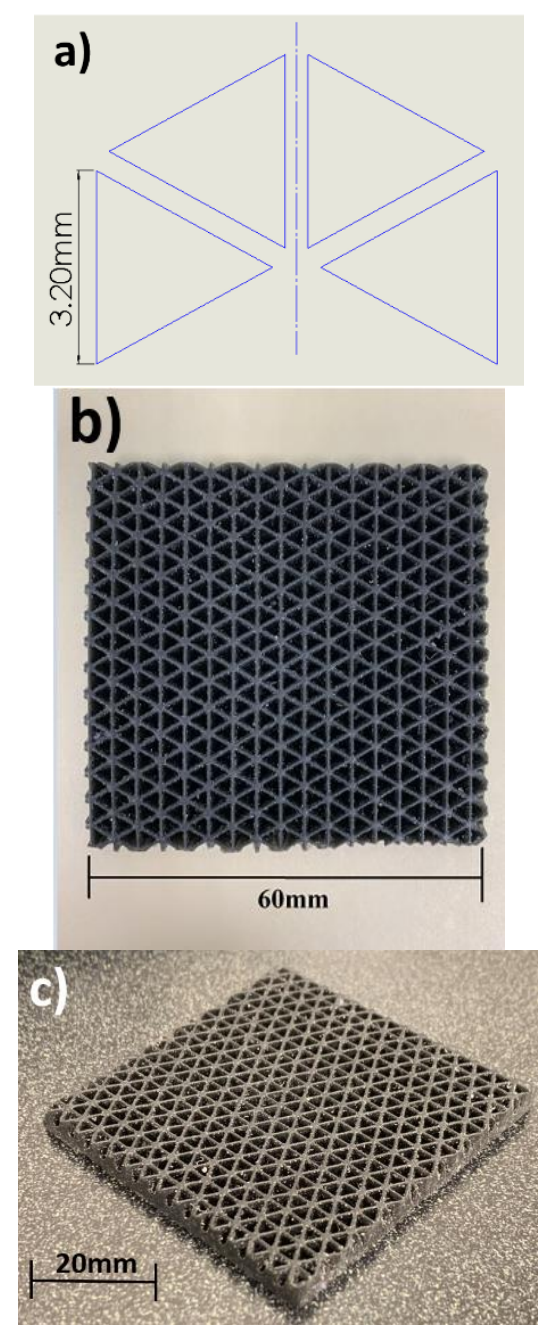

Figure 2.1 Metamaterial Physical Design a) Triangular Patterned Unit Cell b) Top View of Metamaterial c) Isometric View of Metamaterial

The base material is made of a hyperelastic material, TangoPlus (Stratasys, Ltd, Eden Prairie, $M N)$. All samples were additively manufactured using an Object Connex 350 3D printer. The base material is highly flexible and has a similar feel and appearance as rubber. For this study, the draft angles were limited to $0,1.9,4.1,6.6,9.7,14.5$, and 27.5 degrees. The prosthetist that aided in the design of this metamaterial used previous experience to select draft angles that gave approximately equal distant hardness values, presented in Table 2.1, between some of the softest (low density Poron ${ }^{\circledR}$ ) and hardest $\left(\right.$ Plastazote $^{\circledR}$ ) 
currently used orthotic materials [59]. The hardness gaps between materials could be reduced by introducing a larger set of investigated draft angles. The material samples are referenced as $\mathrm{DA}_{00}, \mathrm{DA}_{02}, \mathrm{DA}_{04}, \mathrm{DA}_{06}, \mathrm{DA}_{09}, \mathrm{DA}_{14}$, and $\mathrm{DA}_{27}$ with the two digits subscript to "DA" corresponding to the approximate draft angle associated with the patterned cut.

Table 2.1 Shore O Hardness Values for Each Metamaterial Variation

\begin{tabular}{c|cccccccc}
\hline Draft Angle & $\mathrm{DA}_{00}$ & $\mathrm{DA}_{02}$ & $\mathrm{DA}_{04}$ & $\mathrm{DA}_{06}$ & $\mathrm{DA}_{09}$ & $\mathrm{DA}_{14}$ & $\mathrm{DA}_{27}$ & Base \\
$\begin{array}{c}\text { Shore O } \\
\text { Hardness }\end{array}$ & 13.7 & 16.5 & 20.3 & 23.0 & 28.5 & 30.2 & 33.9 & 44.3 \\
\hline
\end{tabular}

This metamaterial was originally designed to mimic changes in material hardness that could be linked to changes in the metamaterial's mechanical behavior. The material properties of this metamaterial, which are characterized by both the material composition and physical structure, cannot be strictly defined by properties such as hardness or stiffness because hardness can only measure the properties of a base material and stiffness is generally limited to a linear elasticity, neither of which captures the full scope of the mechanical response of the metamaterials. The correlation between hardness and changes in mechanical response are not directly proportional, but, in a relative sense, a low hardness value correlates to a smaller required force to reach a desired material deformation and vice-versa [59]. The change in draft angle alters fill volumes of the voids, as seen in Figure 2.2, and thus the thickness of the walls between each cell, which in turn affects the mechanical behavior of the material. The draft angle for each side of the triangle of the individual triangles is equal. The draft angle shown on the left side of each image of Figure 2.2 appears to be larger than the draft angle listed because of the orientation of the section 
view these images were taken from. The angles on the left are not a representation of the true draft angle for the individual triangles within the unit cell. Instead, those are represented by the draft angles that are specifically called out in red in Figure 2.2.
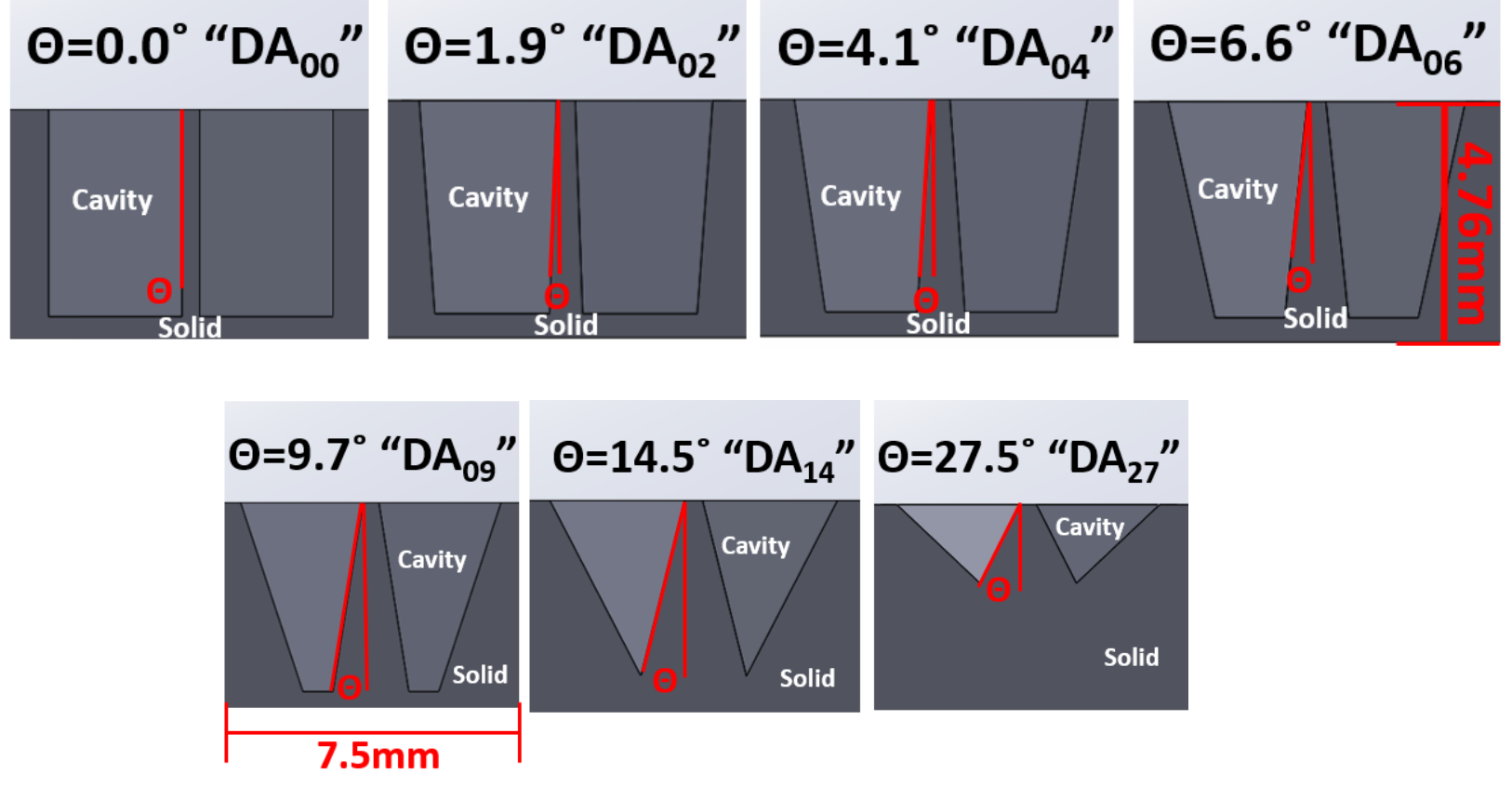

Figure 2.2. Wall Thickness Variances of Each Draft Angle

\subsection{Material Properties}

To develop material models for the metamaterial variations, $60 \mathrm{~mm}$ by $60 \mathrm{~mm}$ samples $(n=3)$ of each draft angle variant metamaterial were tested in uniaxial compression, using an ADMET eXpert 5601 testing system (ADMET, Norwood, MA) (Figure 2.3). The compression plate measured $203 \mathrm{~mm}$ by $203 \mathrm{~mm}$ and therefore covered the entirety of the testing sample. The testing followed ASTM D575 standards for all components of rubber material testing besides the thickness of the test specimen. ASTM D575 calls for a slab approximately $13 \mathrm{~mm}$ in thickness. However, the metamaterial in this study is designed to be approximately $4.76 \mathrm{~mm}$ in thickness for use in the inlay and therefore the deflection rate 
of the plate was altered from $12 \mathrm{~mm} / \mathrm{min}$ to $4.4 \mathrm{~mm} / \mathrm{min}$ to ensure reliable and repeatable experimental methods. Three samples of each material were tested to account for material property variability that can arise over time from this composition [59]. Sanders et al. [19] utilized this quasi-static method of material compression testing with prosthetic liner materials and determined that these methods were viable to represent the in vivo conditions a liner would undertake.

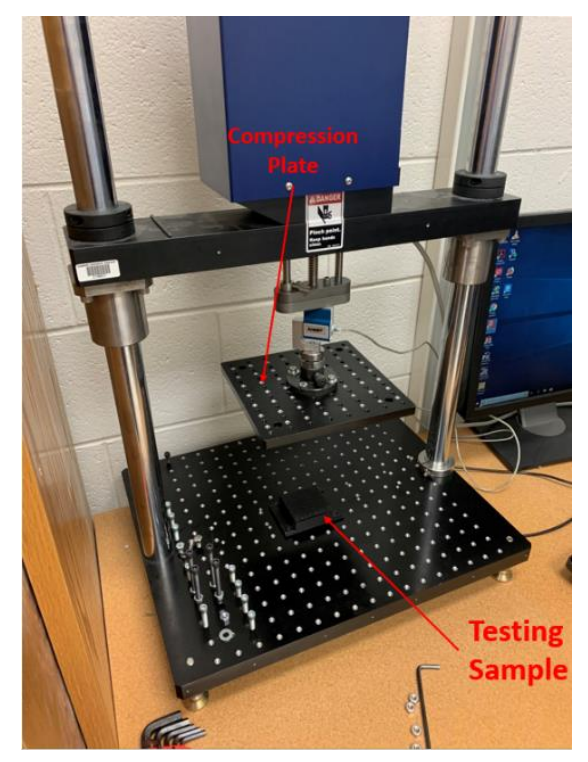

\section{Figure 2.3. ADMET eXpert 5601 Testing System}

The stress-strain curves, seen in Figure 2.4, show the variable material properties that can be achieved through the altering of the draft angle. Each material shows hyperelastic behaving material properties. The smaller wall thicknesses that arise from the lower draft angles lead to a more drastic buckling during compression. This is represented by the noticeable reduction in slope between $40 \%$ and $60 \%$ strain of the stress-strain curves for $\mathrm{DA}_{00}, \mathrm{DA}_{02}, \mathrm{DA}_{04}$, and $\mathrm{DA}_{06}$. 


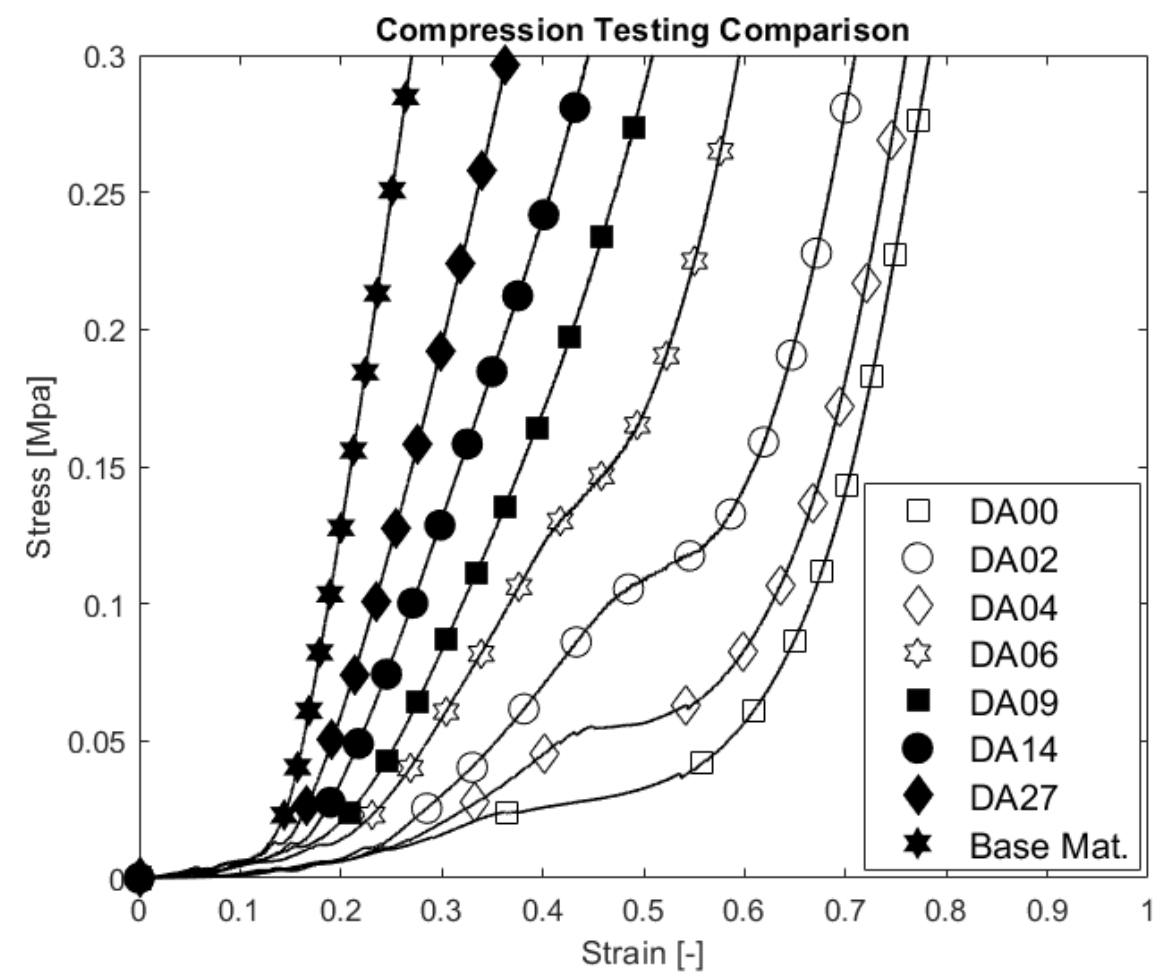

Figure 2.4. Stress-Strain Results of Experimental Compression Testing

A material model was needed in order to provide simplified representations of the experimental stress-strain curves. A range of hyperelastic material models can be used to model the non-linear deformation of a material including Neo-Hookean, Blatz-Ko Mooney-Rivlin 2,3,5, \& 9 Parameter, Polynomial $1^{\text {st }}-3^{\text {rd }}$ Order, and Yeoh $1^{\text {st }}-3^{\text {rd }}$ order, along with others. The hyperelastic materials are described through a strain-energy function. The strain-energy density can be used to derive the relationship between the stresses and strains of a material during deformation. The non-linear relationship between the stresses and strains are defined through a series of material parameters. High order material models have more material parameters which may more accurately describe the stress-strain relationship, but they also increase the complexity of the material model. The 
material parameters are selected so that the model best matches the experimental stressstrain results [60]. For this research, the selected material model needed to accurately represent the experimental data while limiting the number of material parameters. Using a tool within ANSYS® Academic Research Mechanical, Release 19.2, the accuracy and simplicity of several material models were tested, and a Yeoh $3^{\text {rd }}$ order representation was selected to model the compression testing results. The three Yeoh material coefficients $\left(\mathrm{C}_{10}, \mathrm{C}_{20}\right.$, and $\left.\mathrm{C}_{30}\right)$ were found such that, when plugged into $\mathrm{Eq} 2.1$ [60], the model best matched the compression testing data of the given material.

$$
\sigma=\sum_{\mathrm{i}=1}^{3} 2 \mathrm{iC}_{\mathrm{i} 0}\left((1+\varepsilon)-(1+\varepsilon)^{-2}\right)\left((1+\varepsilon)^{2}+\left(2(1+\varepsilon)^{-1}-3\right)\right)^{\mathrm{i}-1}
$$

Table 2.2 lists the material coefficients that correspond to the respective compression testing data. Third order graphical representations, using Eq. 2.1, of each material can be seen in Figure 2.5.

Table 2.2 Yeoh $3^{\text {rd }}$ Order Material Coefficients of Each Metamaterial and Base Composite Material

\begin{tabular}{cccc}
\hline & $\mathrm{C}_{10}[\mathrm{~Pa}]$ & $\mathrm{C}_{20}[\mathrm{~Pa}]$ & $\mathrm{C}_{30}[\mathrm{~Pa}]$ \\
\hline $\mathrm{DA}_{00}$ & 3225 & 95778 & 32987 \\
$\mathrm{DA}_{02}$ & 646 & $1.599 \mathrm{E} 5$ & 42500 \\
$\mathrm{DA}_{04}$ & 1846 & $1.877 \mathrm{E} 5$ & $2.855 \mathrm{E} 5$ \\
$\mathrm{DA}_{06}$ & 1760 & 79549 & -7016 \\
$\mathrm{DA}_{09}$ & 3260 & 66206 & -11759 \\
$\mathrm{DA}_{14}$ & 839 & 23329 & 2455 \\
DA $_{27}$ & 1271 & 6326 & 9544 \\
Base Mat. & 446 & 88326 & $2.510 \mathrm{E} 6$ \\
\hline
\end{tabular}




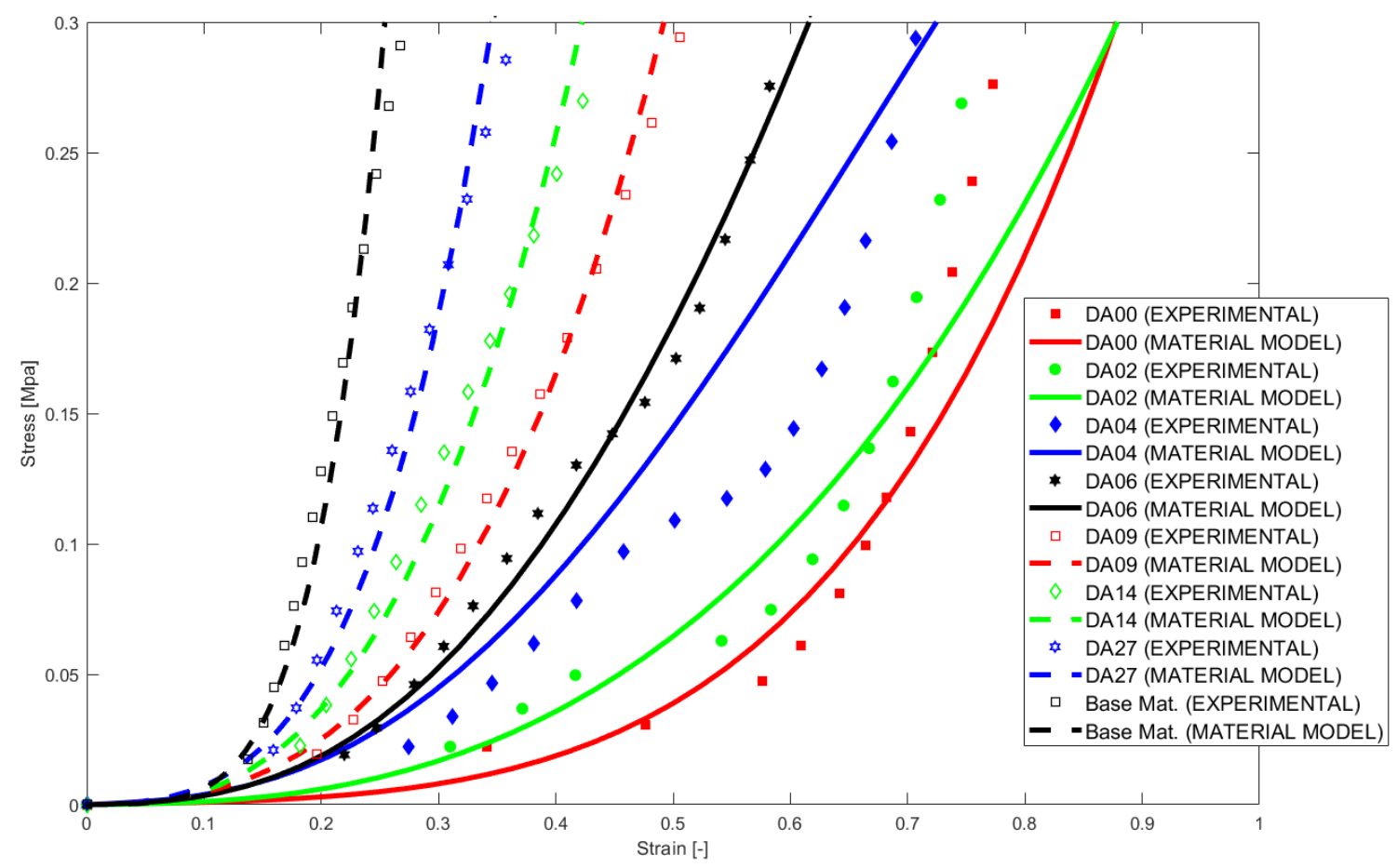

Figure 2.5 Stress-train Comparison Between Experimental Compression Results and Yeoh $3^{\text {rd }}$ Order Material Coefficients

The calculated Yeoh $3^{\text {rd }}$ order coefficients and model were determined to be reasonable approximations of the metamaterials for this research (Table 2.3 lists $\mathrm{R}^{2}$ values). The $\mathrm{R}^{2}$ values represent how closely the experimental compression testing results fit with the Yeoh $3^{\text {rd }}$ order representation.

Table 2.3 $\mathbf{R}^{2}$ Values for Material Coefficients with Respect to Compression Testing Results

\begin{tabular}{cccccccc}
\hline $\mathrm{DA}_{00}$ & $\mathrm{DA}_{02}$ & $\mathrm{DA}_{04}$ & $\mathrm{DA}_{06}$ & $\mathrm{DA}_{09}$ & $\mathrm{DA}_{14}$ & $\mathrm{DA}_{27}$ & Base Mat. \\
\hline 0.908 & 0.904 & 0.921 & 0.986 & 0.990 & 0.978 & 0.974 & 0.947 \\
\hline
\end{tabular}

A limitation for this third order representation is properly representing the buckling experienced by lower draft angle materials, as represented by $\mathrm{DA}_{00}, \mathrm{DA}_{02}$, and $\mathrm{DA}_{04}$ in Figure 2.5. These materials have a lower $\mathrm{R}^{2}$ value because the drastic buckling reaction cannot be properly represented using a third order representation. Materials with larger 
draft angles, like $\mathrm{DA}_{09}$ and $\mathrm{DA}_{14}$, are not subject to as severe wall buckling which means they can more accurately represented using third order coefficients, which is apparent by the larger $\mathrm{R}^{2}$ values. Future work could develop a better material model which captures this buckling at lower draft angles. 


\section{CHAPTER THREE \\ METHODS}

\subsection{FEA Set Up}

With the material properties of the metamaterial established, the next step was to validate the effectiveness of using the metamaterial as an interface material between the residual limb and prosthetic socket to reduce peak pressure gradients (PPG) on the residual limb. As previous stated, in vivo testing of amputees can be very expensive and time consuming [51], therefore finite element analysis (FEA) was used to validate the metamaterials pressure offloading effectiveness. The FEA was to mimic the in vivo conditions of a transtibial amputee. Two different limb shapes were evaluated. The limb models were taken from 3D scans of two deidentified transtibial amputees. The first amputee with limb shape (L1) was approximately a 180-pound, 5-ft 6-in transtibial amputee requiring pressure offloading at the fibular head (FH). The second limb model (L2) came from an approximately 240-pound, 6-ft 4-in transtibial amputee, requiring offloading at the patellar tendon (PT) and tibial end (TE). Both limb models had a conical shape with no abnormal protrusions.

Implicit FEA runs modeled each limb under the following six conditions to compare the peak stress (PS) and peak pressure gradient (PPG) on the surface of the residual limb:

1. No Liner

2. A Homogenous Silicone Gel Liner

3. An inlay with the prosthetist's prescribed material properties and layout

4. An inlay with the prosthetist's prescribed material layout and optimized material properties 
5. An inlay with the prosthetist's prescribed material properties and optimized material layout

6. An inlay with optimized material properties and layout

The material properties of the metamaterial inlays are represented by the Yeoh $3^{\text {rd }}$ Order hyper elastic material model. The silicone gel liner is one of the most popular current liner solutions for transtibial amputees and provides an appropriate comparison target for the metamaterial inlay [20].

The tibia and fibula models were sized, formed, and placed within the limb models based on recommendations from McGrath et al [38]. A bone cavity was formed inside the limb using SOLIDWORKS 2018. The inlay was constructed by isolating the exterior surface of the limb corresponding to the inlay shape and extruding it approximately $4.76 \mathrm{~mm}$ in the normal direction to the residual limb. This inlay model creation method ensures that the inlay and limb remain flush against each other to mimic the interaction of an in vivo limb and inlay. The shapes of the inlays were derived from a clinical prosthetist's recommendation. Unlike common liners that wrap around the entirety of the residual limb, the inlay shape is meant to specifically target the areas that require offloading. The prosthetic socket was formed by scaling the limb to envelop the entire inlay and limb. A limb cavity was formed using SOLIDWORKS 2018 to ensure that the inlay and limb remain flush against the socket, creating a Total Surface Bearing socket (TSB). Zheng et al. [39] implemented a similar method and yielded accurate results. This process was repeated for both limb shape simulations. Figure 3.1 shows the difference between the models used for the two limb shapes. The inlay shapes differ between the two models based on the 
offloading regions. To simplify the analysis for L1, the model was reduced to target just the areas around the FH (Figure 3.2). This simplification drastically reduces the computational time of the simulation. The FEA solution could not compute for a reduced L2 model and therefore the full limb model was necessary.
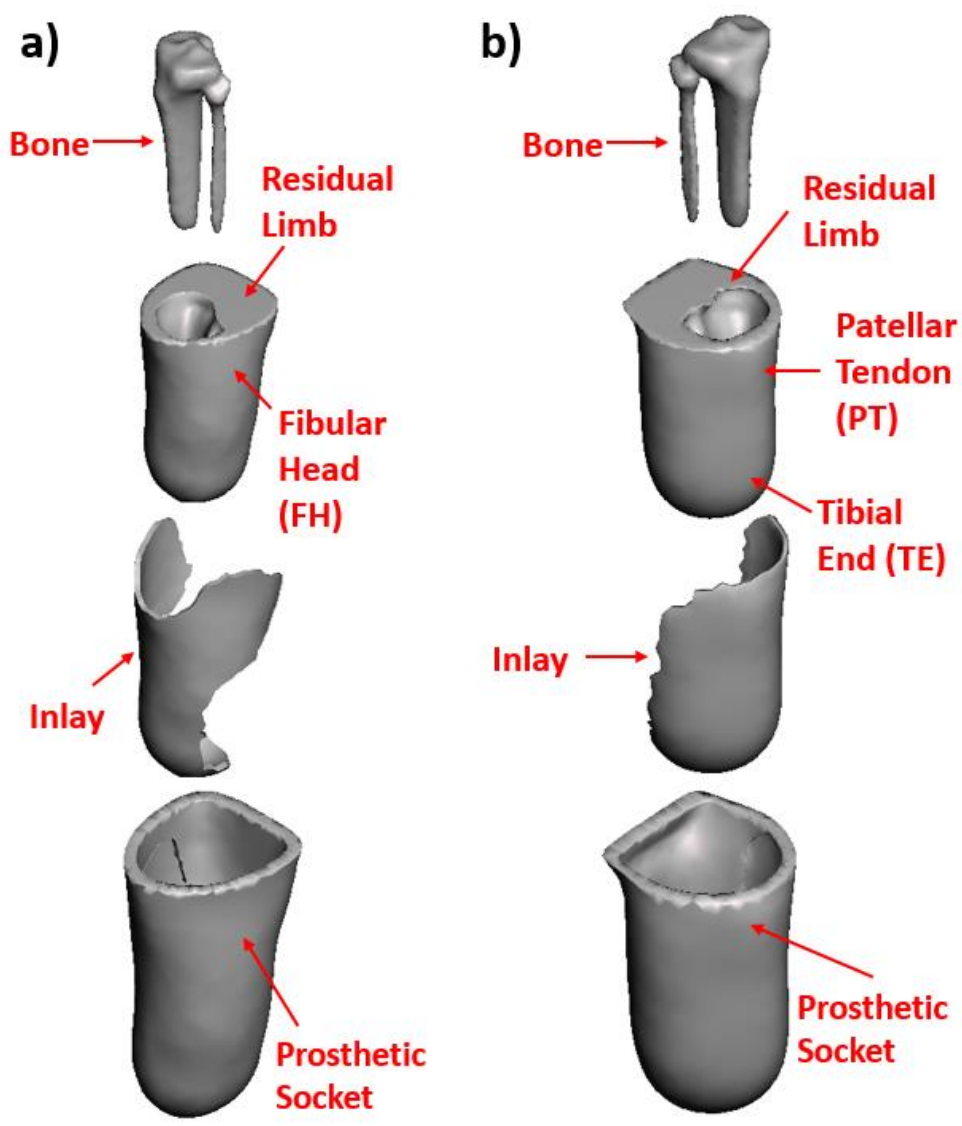

Figure 3.1 FEA Models Exploded a) L1 b) L2 (The top surface of the limb and socket have been removed for clarity) 


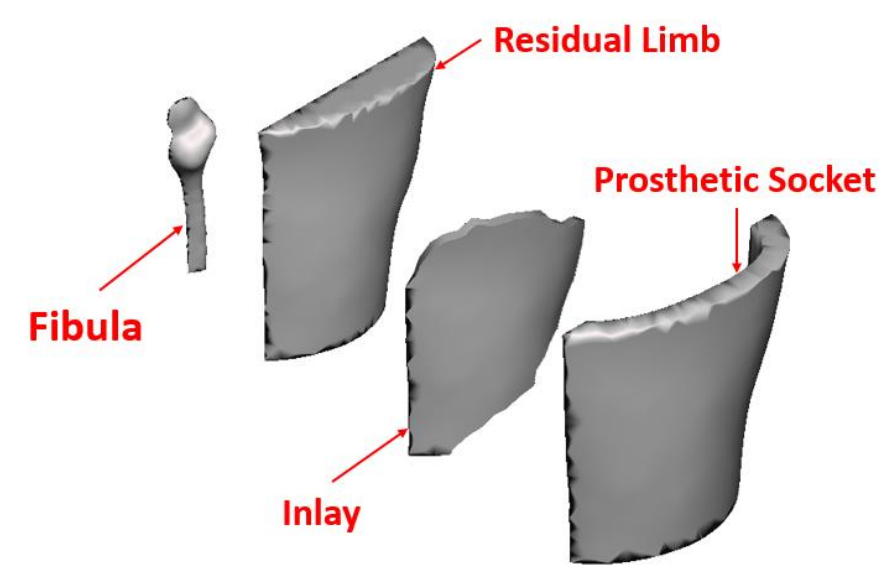

Figure 3.2 Isolated FH Exploded View for Limb Shape 1

The tibia and fibula models were modeled as a linear elastic material with an elastic modulus of $15 \mathrm{GPa}$ and Poisson's ratio of 0.3 [25]. The prosthetic socket was modeled as polypropylene with a linear elastic modulus of $1.5 \mathrm{GPa}$ and Poisson's ratio of 0.3 [25]. The limb model was modeled homogenously as soft tissue. The hyperelastic material properties of soft tissue can be modeled using Yeoh $3^{\text {rd }}$ order coefficients with $C_{10}=$ $0.004154 \mathrm{MPa}, \mathrm{C}_{20}=0.050753 \mathrm{MPa}, C_{30}=-0.013199 \mathrm{MPa}$ [61]. For the "No Inlay" conditions, the inlay was modeled as polypropylene. Several studies have shown that silicone liners can be accurately modeled linearly with a modulus of elasticity of $0.35 \mathrm{MPa}$ and Poisson's ratio of 0.3 [33]

Based on subject matter expertise and patient input, the prosthetists selected the metamaterial variations and their corresponding layouts to design the inlay. The prosthetists had not worked with this metamaterial before and therefore did not have experience to help guide the prescription. 
For L1, the practitioner initially prescribed an inlay (Figure 3.3) that included three materials $\left(\mathrm{DA}_{09}, \mathrm{DA}_{14}\right.$, and $\left.\mathrm{DA}_{27}\right)$ with circular offloading of set radii around the fibular head $(\mathrm{FH})$. The prescription set $\mathrm{DA}_{09}$ as an internal circular region that transitions to a region of $\mathrm{DA}_{14}$ internally and externally bounded by the dimensions seen in Figure 3.3. The remaining portions of the inlay were set as $\mathrm{DA}_{27}$. The regions of $\mathrm{DA}_{09}, \mathrm{DA}_{14}$, and $\mathrm{DA}_{27}$ set by the dimensions in Figure 3.3 are referenced as the "FH Inner Material", "FH Middle Material", and "FH Outer Material", respectively, in later optimization results for the FH.

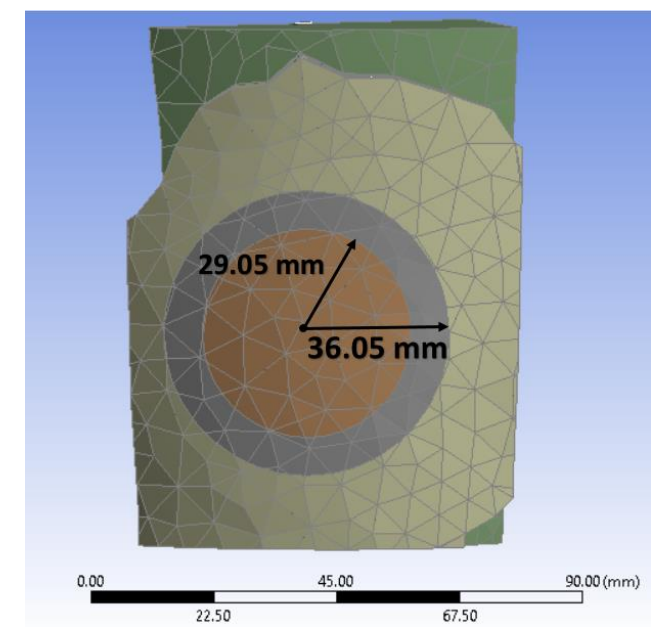

Figure 3.3 Limb Shape 1 Prosthetist's Prescribed Inlay (Prosthetic socket removed for clarity)

For L2, the initially prescribed inlay (Figure 3.4) targeted the patellar tendon (PT) and tibial end (TE) as regions of offloading. The patellar tendon only requires sections of $\mathrm{DA}_{14}$ and $\mathrm{DA}_{27}$. The $\mathrm{DA}_{14}$ region is set by the parameters in Figure 3.4A. This offloading mainly targets the PT through the use of an ellipse layout of $\mathrm{DA}_{14}$ and extends down to the TC. The regions of $\mathrm{DA}_{14}$ and $\mathrm{DA}_{27}$ set by the dimensions in Figure 3.4A are referenced as the "PT Inner Material" and "PT Outer Material", respectively, in later optimization results for the PT. The TE prescription includes $\mathrm{DA}_{09}, \mathrm{DA}_{14}$, and $\mathrm{DA}_{27}$. The internal and external 
ellipses are bounded by the dimensions in Figure 3.4B. The internal and external ellipses were made of $\mathrm{DA}_{09}$ and $\mathrm{DA}_{14}$, respectively. The remaining regions of the inlay not specified by a dimension are represented by $\mathrm{DA}_{27}$. The regions of $\mathrm{DA}_{09}, \mathrm{DA}_{14}$, and $\mathrm{DA}_{27}$ are referenced as the "TE Inner Material", "TE Middle Material", and "TE Outer Material", respectively, in later optimization results for the TE. It should be noted that, within the FEA, the metamaterials are represented by solid bodies assigned the Yeoh $3^{\text {rd }}$ order coefficients presented in Table 2.2.

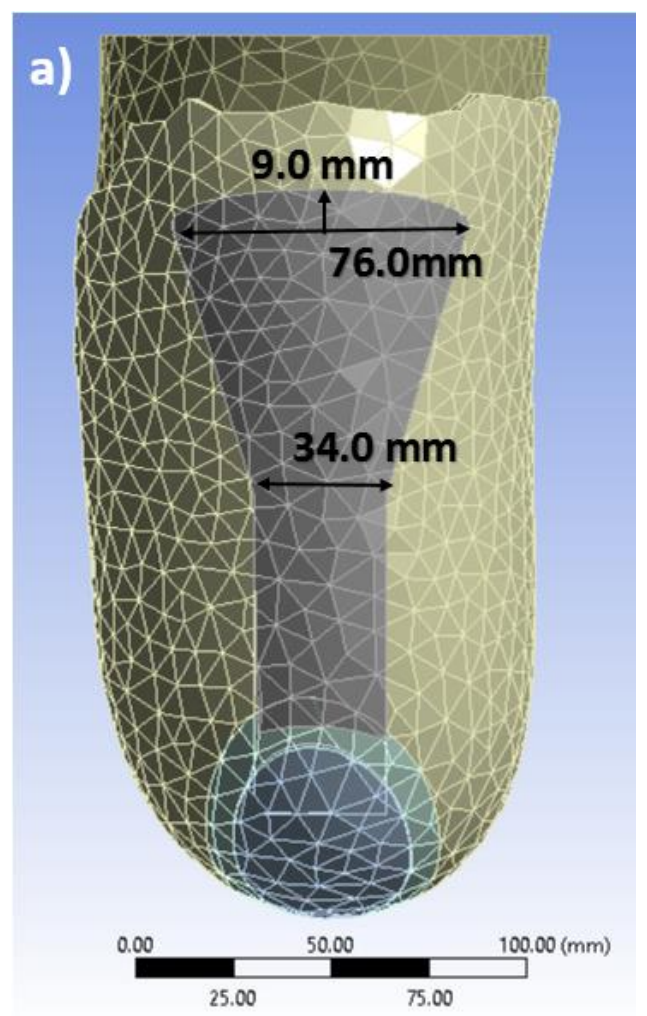




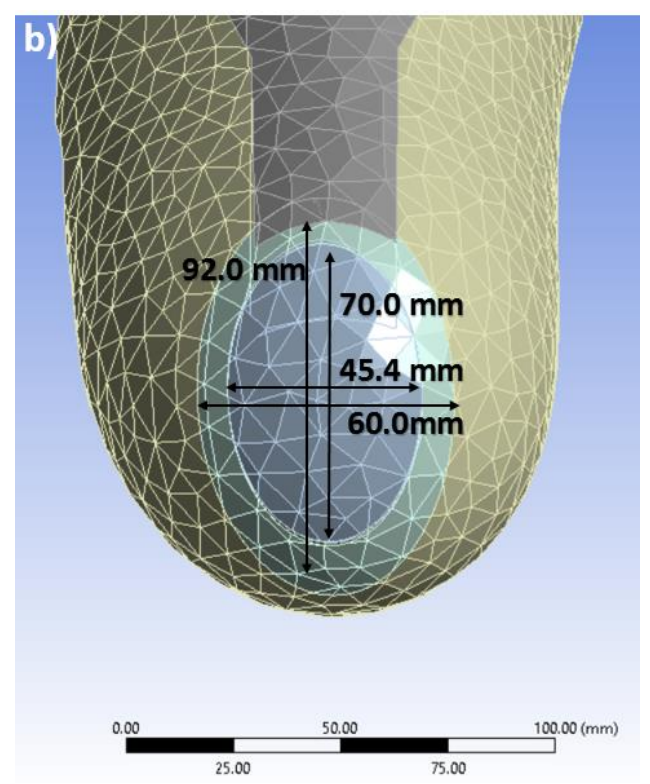

\section{Figure 3.4. Limb Shape 2 Prosthetist's Prescribed Inlay A) Targeting the Patellar Tendon b) Targeting the Tibial End (Prosthetic socket removed for clarity)}

These inlay material layouts were only utilized for the "Prosthetist Prescription" and “Optimized Material Properties" simulations. A further description of the material layout for the simulations that involve layout optimization can be found starting in Section 3.2.2.

For all simulations, the exterior surface of the prosthetic was held as the fixed support. For L1, a load of $60 \mathrm{~N}$ was applied to the flat face of the isolated fibula, represented in Figure 3.5. L2 was loaded with $1090 \mathrm{~N}$ of compression and $205 \mathrm{~N}$ of anterior-posterior shear, represented in Figure 3.6. These loading conditions were set to mimic the largest loading conditions the limb might experience during the gait cycle given the weight of the subject [38]. This quasi-static loading representation of the dynamic nature of an amputee in the gait cycle has been validated by Faustini et al. [41] who utilized quasi-static loading conditions derived from experimentally measured ground reaction forces to mimic in vivo testing within FEA. 

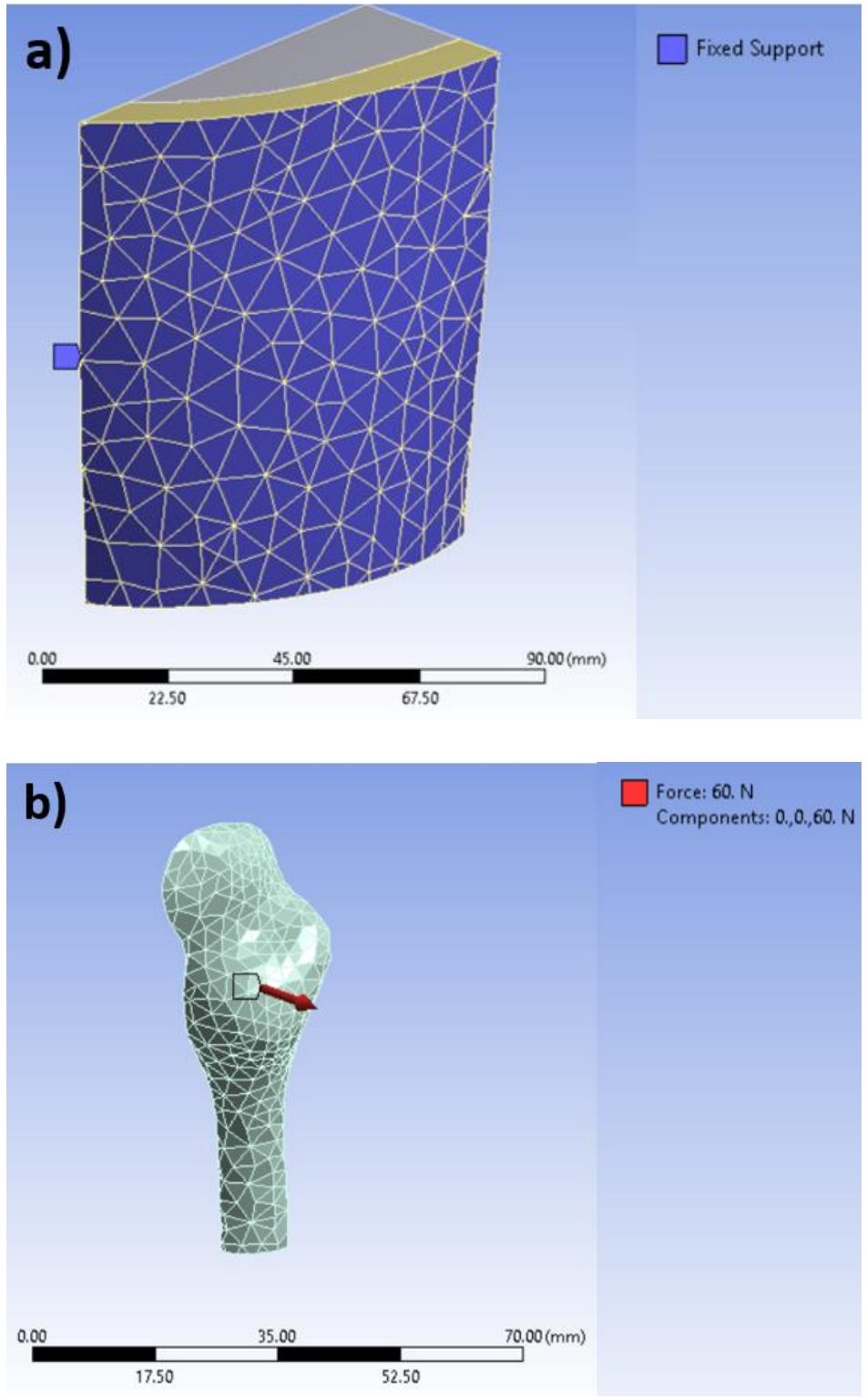

Figure 3.5 FEA Conditions for L1 a) Fixed Support on Prosthetic Socket b) Loading Condition of FH 

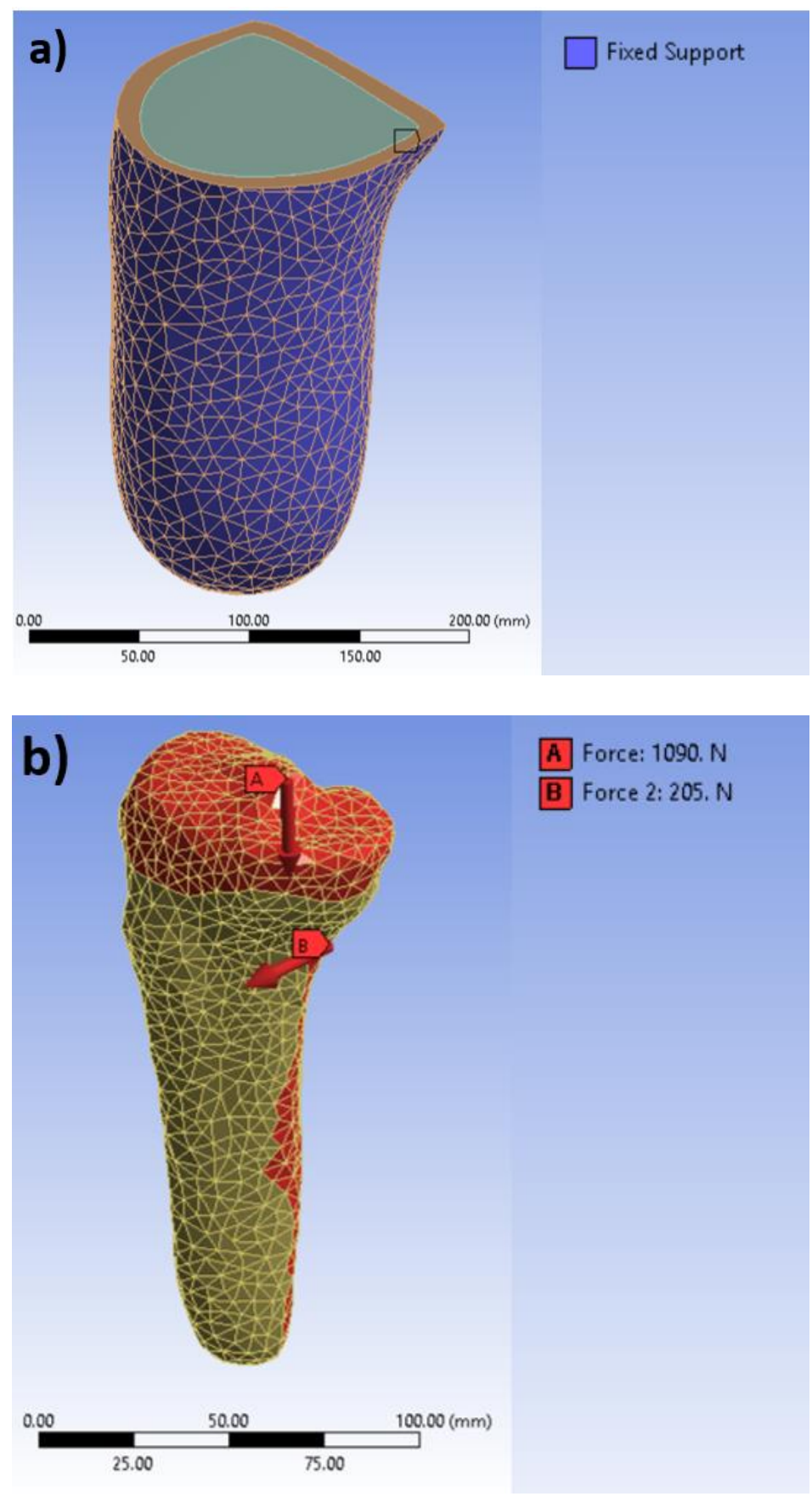

Figure 3.6 FEA Conditions for L2 a) Fixed Support on Prosthetic Socket b) Loading Conditions of Bone 
The coefficient of friction (COF) between the limb and socket, as well as the inlay and socket, were set to $\mu=0.5$. The COF between the inlay and limb is approximately 2.0 and therefore were modeled as bonded [22]. A mesh convergence study (Figure 3.7) was run on the No Inlay condition on L1 and L2 and a maximum mesh element size of 2.0mm was deemed the appropriate selection for both models.

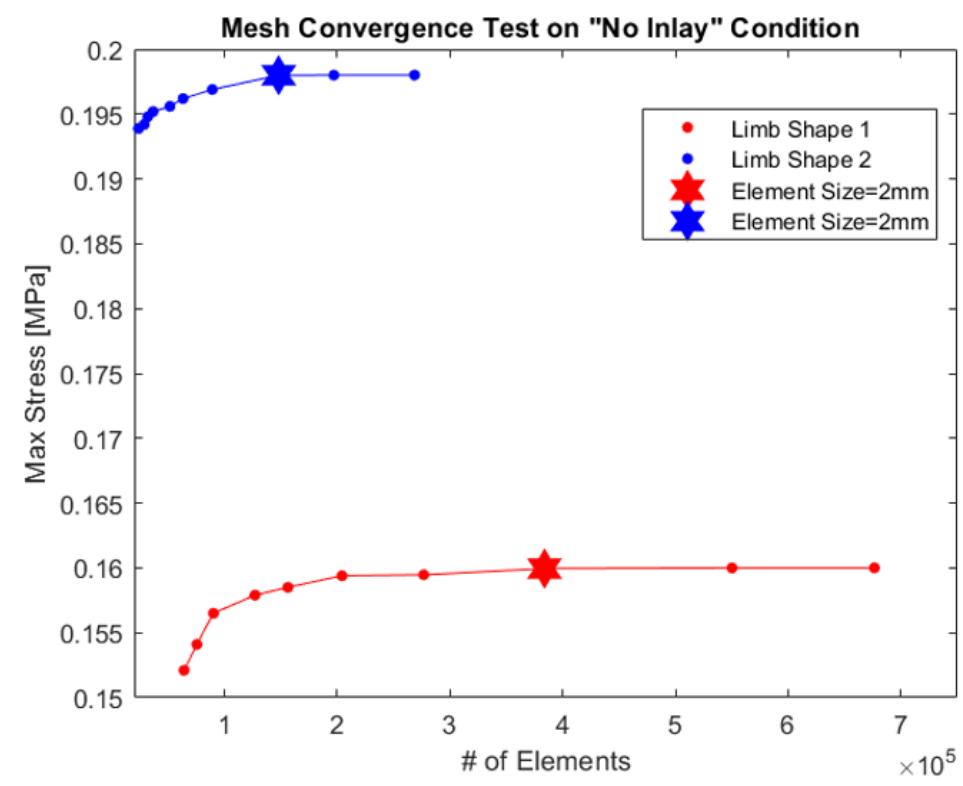

Figure 3.7 Mesh Convergence Study Results

This element size ensures that even the most finite critical components, such as the normal direction of the thin inlay (Figure 3.6), have enough mesh nodes to accurately model the variation in physical phenomenon. The mesh size being the same for both models is validated because the inlay thickness is the same for both models. 


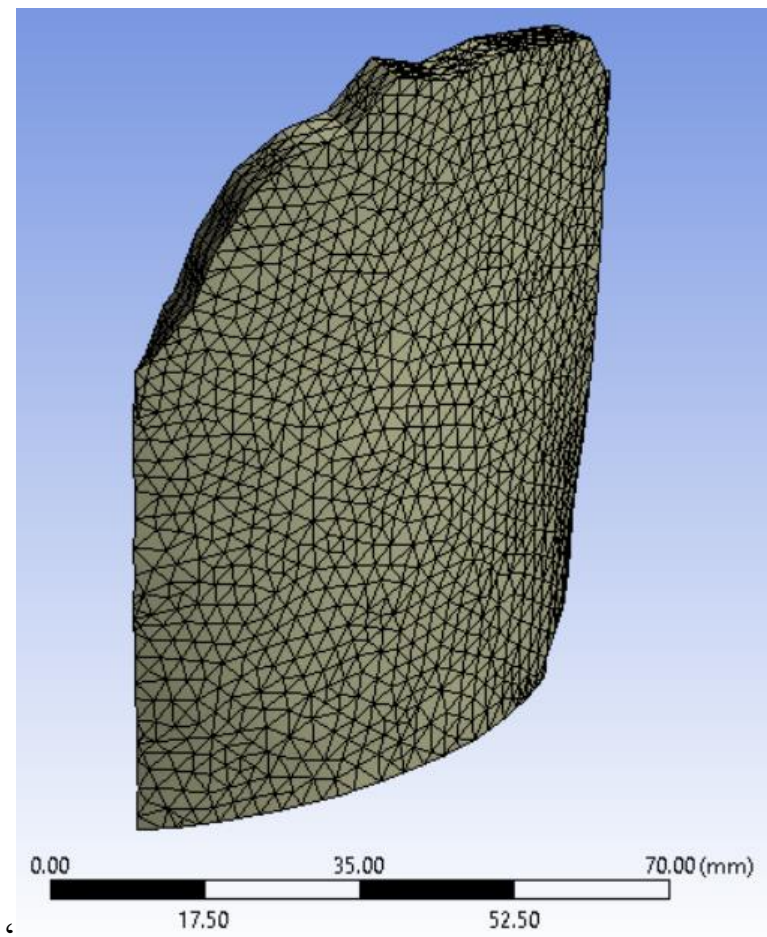

\section{Figure 3.8 Meshed Inlay Model for Limb Shape 1}

\subsection{Optimization Methods and Results}

In an attempt to further investigate the pressure offloading capabilities of the metamaterial inlay, three optimization conditions were individually applied to the inlays for both limb models.

1. Optimizing the material properties while utilizing a prosthetist recommended material layout.

2. Optimizing the material layout while utilizing the prosthetist prescribed material properties.

3. Optimizing both the material properties and the material layout.

Reduction of peak pressure gradient (PPG) on the limb surface is the goal of the optimization. To minimize the PPG, an ideal liner would have a homogenous distribution 
of stress across the residual limb, which would result in the max stress being equal to the average stress on the limb. Therefore, the optimization objective is to minimize the difference between the max and average stress on the limb surface, as shown in equation 3.1 .

$$
\text { obj }=\min \mid\left(\text { Stress }_{\max }-\text { Stress }_{\text {avg }}\right) \mid
$$

Since an analytical equation between the material coefficients and the objective is not available, we choose to use a gradient free optimization algorithm, although other possibilities can be envisioned, such as using a surrogate model. An adaptive multiobjective method, a variant of the popular NSGA-II (Non-dominated Sorted Genetic Algorithm II) [62], was used.

\subsubsection{Material Property Optimization}

The material property optimization of the inlay utilizes the material layouts established in Figures 3.3 and 3.4. This prosthetist prescribed layout calls for three materials (9 coefficients) to offload the fibular head (FH) for limb shape 1 (L1), three materials (9 coefficients) to offload the tibial end (TE) for limb shape 2 (L2), and two materials (6 coefficients) to offload the patellar tendon (PT) for L2. The three Yeoh material coefficients of each material were set as the optimization parameters. Optimizing the material coefficients is meant to act as a representation of optimizing the draft angle of the metamaterial. The idea of altering key parameters to guide the redesigning of unit cells was similar to the one used by Satterfield et al. [63] who showed that altering parameters that represent the physical response of a unit cell can lead to similar results as altering the unit cell directly. The optimization of the material properties is described below. 


$$
\text { Obj }=\min \mid\left(\text { Stress }_{\max }-\text { Stress }_{\text {avg }}\right) \mid
$$

Design Variables: $C_{10-\text { Inner }}, C_{10-\text { Middle }}, C_{10-\text { outer }}, C_{20-\text { Inner }}, C_{20 \text {-Middle }}, C_{20-\text { outer }}$ $C_{30-\text { Inner }}, C_{30-\text { Middle }}, C_{30-\text { outer }}$

Subject To

$$
\begin{aligned}
& 0 P a<C_{10-\text { Inner }}, C_{10-\text { Middle }}, C_{10-\text { outer }}<1 \mathrm{E} 4 \mathrm{~Pa} \\
& 0 \mathrm{~Pa}<C_{20-\text { Inner }}, C_{20 \text {-Middle }}, C_{20-\text { outer }}<5 \mathrm{E} 5 \mathrm{~Pa} \\
& 0 \mathrm{~Pa}<C_{30-\text { Inner }}, C_{30-\text { Middle }}, C_{30-\text { outer }}<5 \mathrm{E} \mathrm{Pa}
\end{aligned}
$$

The bounds on the coefficients were set based on the original values of $\mathrm{C}_{10}, \mathrm{C}_{20}$, and $\mathrm{C}_{30}$ from Table 2.2. For the optimization of the two materials at the PT for L2, only the coefficients corresponding to the "Middle" and "Outer" material were used. The optimized material coefficients are shown in Tables 3.1 and 3.2.

Table 3.1 Optimized Yeoh $3^{\text {rd }}$ Order Material Coefficients At the Fibular Head [FH]

\begin{tabular}{lccc} 
& FH Inner Material & FH Middle Material & FH Outer Material \\
\hline $\mathrm{C}_{10}[\mathrm{~Pa}]$ & 8988 & 3877 & 4559 \\
$\mathrm{C}_{20}[\mathrm{~Pa}]$ & 5019 & $3.297 \mathrm{E} 5$ & $3.487 \mathrm{E} 5$ \\
$\mathrm{C}_{30}[\mathrm{~Pa}]$ & 16937 & $2.577 \mathrm{E} 5$ & $2.834 \mathrm{E} 5$ \\
\hline
\end{tabular}


Table 3.2 Optimized Yeoh $3^{\text {rd }}$ Order Material Coefficients at the Tibial End [TE] and Patellar Tendon [PT]

\begin{tabular}{ccccc}
\hline Limb Area & Coefficient & $\begin{array}{c}\text { TE Inner } \\
\text { Material }\end{array}$ & $\begin{array}{c}\text { TE Middle } \\
\text { Material }\end{array}$ & $\begin{array}{c}\text { TE } \\
\text { Outer } \\
\text { Material }\end{array}$ \\
\hline \multirow{2}{*}{ TE } & $\mathrm{C}_{10}[\mathrm{~Pa}]$ & 1270 & 3058 & 9699 \\
& $\mathrm{C}_{20}[\mathrm{~Pa}]$ & 17340 & $2.993 \mathrm{E} 5$ & $4.244 \mathrm{E} 5$ \\
& $\mathrm{C}_{30}[\mathrm{~Pa}]$ & 37423 & $2.371 \mathrm{E} 5$ & $4.761 \mathrm{E} 5$ \\
\hline & & PT Inner & & PT Outer \\
& & Material & & Material \\
\hline & $\mathrm{C}_{10}[\mathrm{~Pa}]$ & 7537 & - & 9760 \\
& $\mathrm{C}_{20}[\mathrm{~Pa}]$ & $3.877 \mathrm{E} 5$ & - & $4.904 \mathrm{E} 5$ \\
& $\mathrm{C}_{30}[\mathrm{~Pa}]$ & $3.382 \mathrm{E} 5$ & - & $4.899 \mathrm{E} 5$ \\
\hline
\end{tabular}

The bounds are justified given that the results in Tables 3.1 and 3.2 do not hover near the edges of the parameter bounds. Each optimization was set to run with 25 initial samples and generate 10 updated samples every iteration until the convergence stability percentage of $2 \%$ was met. The optimization was performed using ANSYS direct optimization, and the algorithm was run on Clemson University's Palmetto Cluster using 24 cores with 128 GB of RAM. The optimization at the FH took 120 FEA calls to converge, each taking slightly longer than 5 minutes. The optimization around the TE and PT took 140 and 130 FEA calls, respectively. Each simulation took approximately 18 minutes. The results of Tables 3.1 and 3.2 were used to design the inlays for the "Optimized Material Coefficient" results for both limb shapes. 


\subsubsection{Material Layout Optimization}

The material layout optimization took the dimensions from the prosthetist's prescribed material layout of Figures 3.3 and 3.4 and utilize them as design variables in the optimization problem, as seen in Figures 3.5 and 3.6. The alteration of these design variables changes the areas that each material is present on the inlay.

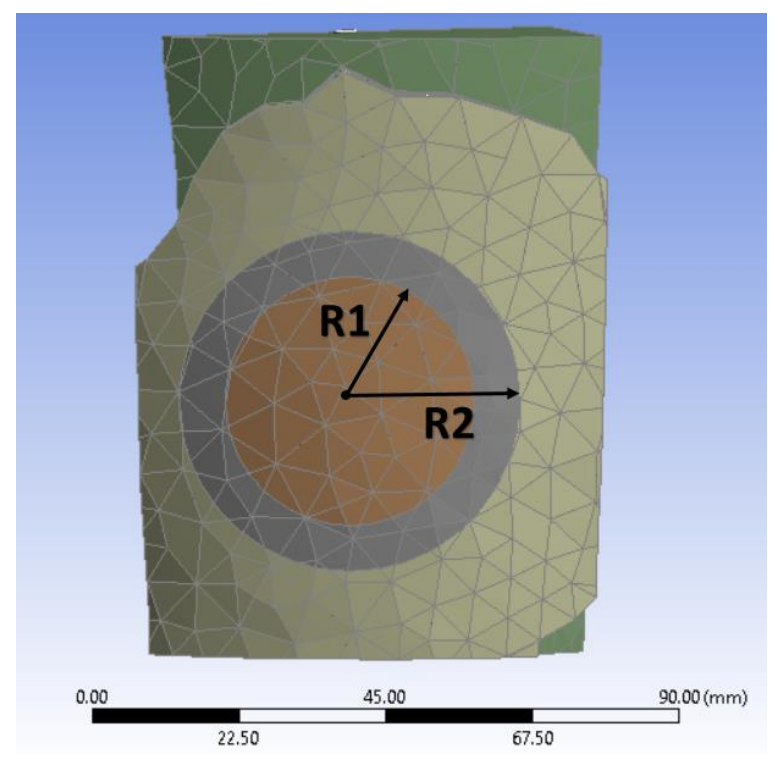

Figure 3.9 Geometric Variables on Inlay at the Fibular Head 

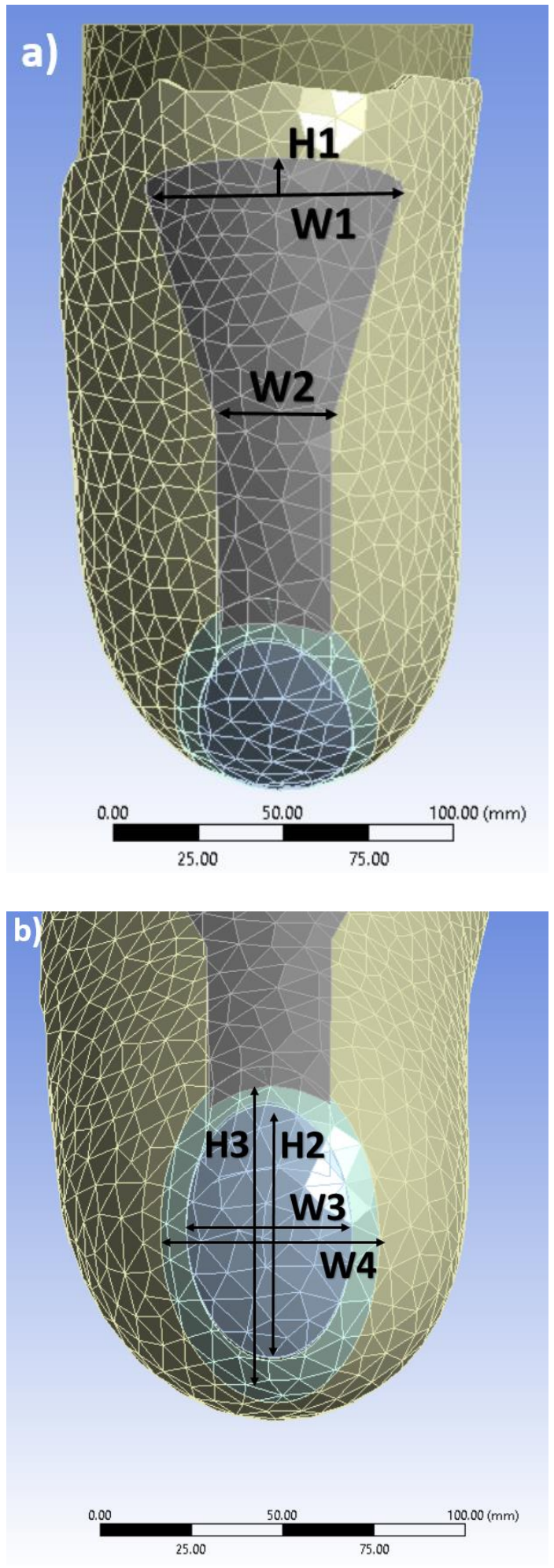

Figure 3.10 Geometric Variables on Inlay at the a) Patellar Tendon b) Tibial End 
Per the prosthetist's recommendation, $\mathrm{DA}_{09}, \mathrm{DA}_{14}$, and $\mathrm{DA}_{27}$ were set as the material representations for this optimization. For limb shape 1 (L1), the internal region bounded by $\mathrm{R} 1$ was modeled as $\mathrm{DA}_{09}$. The middle region bounded internally and externally by $\mathrm{R} 1$ and $\mathrm{R} 2$, respectively, was modeled as $\mathrm{DA}_{14}$. The remaining portions of the inlay for $\mathrm{L} 1$ were modeled as $\mathrm{DA}_{27}$. At the patellar tendon (PT) for limb shape 2 (L2), the region externally bounded by H1, W1, and W2 was modeled as $\mathrm{DA}_{14}$. At the tibial end (TE) for L2, the internal ellipse bounded by $\mathrm{H} 2$ and $\mathrm{W} 2$ was modeled as $\mathrm{DA}_{09}$. The middle ellipse bounded internally by $\mathrm{H} 2$ and $\mathrm{W} 2$ and externally by $\mathrm{H} 3$ and $\mathrm{W} 4$ was modeled as $\mathrm{DA}_{14}$. All regions of the inlay not bounded by one of the dimensions mentioned were modeled as $\mathrm{DA}_{27}$. The layout optimizations for the three limb regions are described below.

$$
O b j=\min \mid\left(\text { Stress }_{\max }-\text { Stress }_{\text {avg }}\right) \mid
$$

1. Fibular Head Design Variables: R1, R2

Subject To

$$
\begin{gathered}
5 \mathrm{~mm}<R 1<22 \mathrm{~mm} \\
11 \mathrm{~mm}<R 2<28 \mathrm{~mm} \\
R 1<R 2
\end{gathered}
$$

2. Patellar Tendon Design Variables: H1, W1, W2 Subject To

$$
\begin{gathered}
2 \mathrm{~mm}<H 1<30 \mathrm{~mm} \\
50 \mathrm{~mm}<W 1<100 \mathrm{~mm} \\
22 \mathrm{~mm}<W 2<33 \mathrm{~mm}
\end{gathered}
$$

3. Tibial End Design Variables: H2, H3, W3, W4

Subject To

$$
\begin{gathered}
40 \mathrm{~mm}<H 2<80 \mathrm{~mm} \\
50 \mathrm{~mm}<H 3<80 \mathrm{~mm} \\
2 \mathrm{~mm}<W 3<20 \mathrm{~mm} \\
30 \mathrm{~mm}<W 4<80 \mathrm{~mm} \\
H 2<H 3 \\
W 3<W 4
\end{gathered}
$$


The bounds at each location were determined based on physical inlay sizing constrains, as well as the prosthetist's initial layout recommendation. The sizing inequalities at the FH and TE were set to ensure that two material regions did not overlap. The optimized layout dimensions are shown in Tables 3.3 and 3.4.

Table 3.3 Optimized Material Layout at the Fibular Head [FH]

\begin{tabular}{cc} 
Dimension & Value $[\mathrm{mm}]$ \\
\hline R1 & 21.8 \\
R2 & 23.5 \\
\hline
\end{tabular}

Table 3.4 Optimized Material Layout at the Patellar Tendon [PT] and Tibial End [TE]

\begin{tabular}{ccc}
\hline Limb Area & Dimension & Value $[\mathrm{mm}]$ \\
\hline \multirow{2}{*}{ PT } & H1 & 2.7 \\
& W1 & 61.3 \\
& W2 & 27.3 \\
\hline \multirow{2}{*}{ TE } & H2 & 68.8 \\
& H3 & 69.4 \\
& W3 & 17.2 \\
& W4 & 52.8 \\
\hline
\end{tabular}

The bounds are justified by the results in Tables 3.3 and 3.4 not hovering near the bounds. The genetic algorithm optimizations were set to run with 15 initially assigned samples and generate 5 updated samples every iteration until convergence. ANSYS direct optimization was again run on Clemson University's Palmetto Cluster. The FH, PT, and TE required 35, 40, and 55 FEA calls respectively. Each FEA call took approximately 5 
minutes for the FH and 18 minutes for the PT and TE. The results from Tables 3.3 and 3.4 were used as the material layouts of the inlays for the "Optimized Material Layout" results.

\subsubsection{Material Property and Layout Optimization}

The final optimization sought to optimize the material properties and layout simultaneously by setting the Yeoh coefficients and layout parameters as the design variables. The optimization set up for each limb region is described below.

$$
\text { obj }=\min \mid\left(\text { Stress }_{\max }-\text { Stress }_{\text {avg }}\right) \mid
$$

Fibular Head Design Variables: R1, R2, $C_{10-\text { Inner }}, C_{10-\text { Middle }}, C_{10-\text { outer }}$

$$
\begin{aligned}
& C_{20-\text { Inner }}, C_{20 \text {-Middle }}, C_{20 \text {-Outer }} \\
& C_{30 \text {-Inner }}, C_{30 \text {-Middle }}, C_{30 \text {-Outer }}
\end{aligned}
$$

Subject To

$$
\begin{gathered}
0 \mathrm{~Pa}<C_{10-\text { Inner }}, C_{10-\text { Middle }}, C_{10-\text {-Outer }}<1 \mathrm{E} 4 \mathrm{~Pa} \\
0 \mathrm{~Pa}<C_{20-\text { Inner }}, C_{20-\text { Middle }}, C_{20 \text {-Outer }}<55 \mathrm{~Pa} \\
0 \mathrm{~Pa}<C_{30-\text { Inner }}, C_{30-\text { Middle }}, C_{30 \text {-outer }}<5 \mathrm{E} 5 \mathrm{~Pa} \\
5 \mathrm{~mm}<R 1<22 \mathrm{~mm} \\
11 \mathrm{~mm}<R 2<28 \mathrm{~mm} \\
R 1<R 2
\end{gathered}
$$

Patellar Tendon Design Variables: H1, W1, W2, $C_{10-\text { Middle }}, C_{10-\text { outer }}$

$$
\begin{aligned}
& C_{20-\text { Middle }}, C_{20 \text {-Outer }} \\
& C_{30 \text {-Middle }}, C_{30 \text {-outer }}
\end{aligned}
$$

Subject To

$$
\begin{gathered}
0 \mathrm{~Pa}<C_{10 \text {-Middle }}, C_{10-\text { outer }}<1 E 4 \mathrm{~Pa} \\
0 \mathrm{~Pa}<C_{20 \text {-Middle }}, C_{20 \text {-Outer }}<5 \mathrm{E} 5 \mathrm{~Pa} \\
0 \mathrm{~Pa}<C_{30-\text { Middle }}, C_{30 \text {-Outer }}<5 \mathrm{E} 5 \mathrm{~Pa} \\
1 \mathrm{~mm}<H 1<30 \mathrm{~mm} \\
50 \mathrm{~mm}<W 1<100 \mathrm{~mm} \\
22 \mathrm{~mm}<W 2<33 \mathrm{~mm}
\end{gathered}
$$

Tibial End Design Variables: $\mathrm{H} 2, \mathrm{H} 3, \mathrm{~W} 3, \mathrm{~W} 4, C_{10-\text { Inner }}, C_{10-\text { Middle }}, C_{10-\text { outer }}$

$$
C_{20-\text { Inner }}, C_{20-\text { Middle }}, C_{20-\text { outer }}
$$$$
C_{30-\text { Inner }}, C_{30-\text { Middle }}, C_{30-\text { Outer }}
$$

Subject To

$$
\begin{aligned}
& 0 \mathrm{~Pa}<C_{10 \text {-Inner }}, C_{10 \text {-Middle }}, C_{10-\text { outer }}<1 \mathrm{E} 4 \mathrm{~Pa} \\
& 0 \mathrm{~Pa}<C_{20 \text {-Inner }}, C_{20 \text {-Middle }}, C_{20 \text {-outer }}<5 \mathrm{E} 5 \mathrm{~Pa} \\
& 0 \mathrm{~Pa}<C_{30 \text {-Inner }}, C_{30 \text {-Middle }}, C_{30 \text {-outer }}<5 \mathrm{E} 5 \mathrm{~Pa}
\end{aligned}
$$




$$
\begin{gathered}
40 \mathrm{~mm}<H 2<80 \mathrm{~mm} \\
50 \mathrm{~mm}<H 3<80 \mathrm{~mm} \\
2 \mathrm{~mm}<W 3<20 \mathrm{~mm} \\
30 \mathrm{~mm}<W 4<80 \mathrm{~mm} \\
H 2<H 3 \\
W 3<W 4
\end{gathered}
$$

The bounds were set based on the rational used for setting the bounds of the previous optimization problems. The lower bound for $\mathrm{H} 1$ at the patellar tendon was reduced in response to the optimized layout result for $\mathrm{H} 1$. The optimization results for the three regions can be found below.

Table 3.5 Optimized Material Coefficients and Layout at the Fibular Head [FH]

\begin{tabular}{cc}
\hline Dimension & Value $[\mathrm{mm}]$ \\
\hline R1 & 10.3 \\
R2 & 11.9
\end{tabular}

\begin{tabular}{cccc}
\hline \multirow{2}{*}{ Coefficient } & FH Inner Material & FH Middle Material & FH Outer Material \\
\hline $\mathrm{C}_{10}$ & 8988 & 3877 & 4559 \\
$\mathrm{C}_{20}$ & 5019 & $3.230 \mathrm{E} 5$ & $3.487 \mathrm{E} 5$ \\
$\mathrm{C}_{30}$ & 16937 & $2.577 \mathrm{E} 5$ & $2.835 \mathrm{E} 5$ \\
\hline
\end{tabular}


Table 3.6 Optimized Material Coefficients and Layout at Patellar Tendon [PT] and Tibial End [TE]

\begin{tabular}{|c|c|c|c|c|}
\hline & Limb Area & Dimension & Value $[\mathrm{mm}]$ & \\
\hline & \multirow{3}{*}{ PT } & $\mathrm{H} 1$ & 1.3 & \\
\hline & & W1 & 60.2 & \\
\hline & & W2 & 22.1 & \\
\hline & \multirow{4}{*}{$\mathrm{TE}$} & $\mathrm{H} 2$ & 69.8 & \\
\hline & & H3 & 92.7 & \\
\hline & & W3 & 41.2 & \\
\hline & & W4 & 85.8 & \\
\hline Limb Area & Coefficient & $\begin{array}{l}\text { PT Inner } \\
\text { Material }\end{array}$ & & $\begin{array}{l}\text { PT Outer } \\
\text { Material }\end{array}$ \\
\hline \multirow{4}{*}{ PT } & $\mathrm{C}_{10}$ & 6024 & & 7453 \\
\hline & $\mathrm{C}_{20}$ & $2.014 \mathrm{E} 5$ & & $2.451 \mathrm{E} 5$ \\
\hline & $\mathrm{C}_{30}$ & $3.320 \mathrm{E} 5$ & & $3.712 \mathrm{E} 5$ \\
\hline & & $\begin{array}{l}\text { TE Inner } \\
\text { Material }\end{array}$ & $\begin{array}{c}\text { TE Middle } \\
\text { Material }\end{array}$ & $\begin{array}{l}\text { TE Outer } \\
\text { Material }\end{array}$ \\
\hline \multirow{3}{*}{$\mathrm{TE}$} & $\mathrm{C}_{10}$ & 7389 & 6206 & 8652 \\
\hline & $\mathrm{C}_{20}$ & 47015 & $1.702 \mathrm{E} 5$ & $2.647 \mathrm{E} 5$ \\
\hline & $\mathrm{C}_{30}$ & 70075 & $4.623 \mathrm{E} 5$ & $3.6454 \mathrm{E} 5$ \\
\hline
\end{tabular}

All the optimizations were set to run with 60 initially assigned samples and generate 15 updated samples every iteration until convergence. ANSYS direct optimization was again tied to Clemson University's Palmetto Cluster. The FH, PT, and TE required 165, 180, and 210 FEA calls, respectively. Each FEA call took approximately 5 minutes for the FH and 18 minutes for the PT and TE. The results of Table 3.5 and 3.6 were used to design the inlay for the "Optimized Material Coefficient and Layout" results. 


\section{CHAPTER FOUR \\ STRESS RESULTS}

With the prosthetist designed and optimized metamaterial inlays designed, the peak stress (PS) and peak pressure gradient (PPG) reduction capabilities of the metamaterial could be determined and compared to "No Liner" and silicone liner conditions. Equivalent Von-Misses stresses on the surface of the residual limb were determined for each inlay condition. Figure 4.1 and 4.2 are examples of the visual representation of the FEA results which were used to determine the PS and PPG for each condition at the fibular head (FH) and the tibial end (TE)/patellar tendon (PT), respectively. The full set of limb surface stress FEA results for each condition can be found in Appendix B.

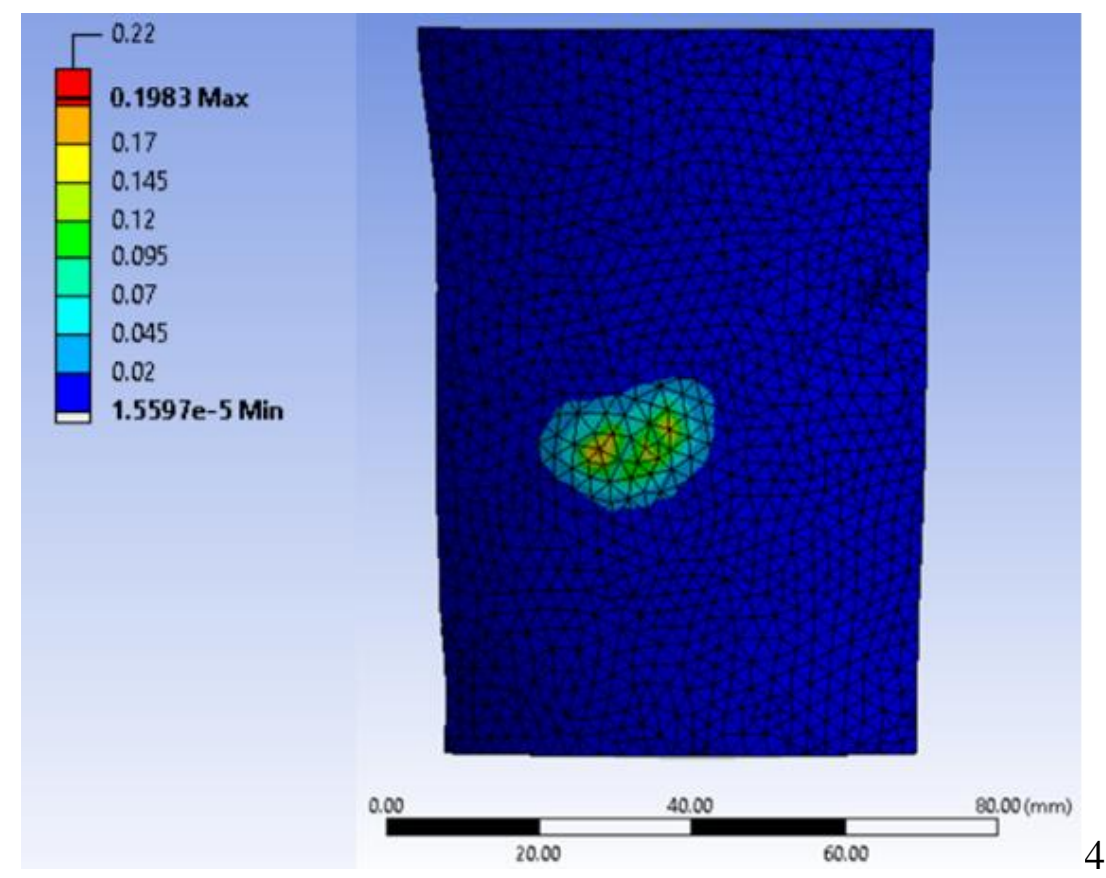

Figure 4.1. Limb Surface Stress for Limb Shape 1 at the Fibular Head [MPa] 


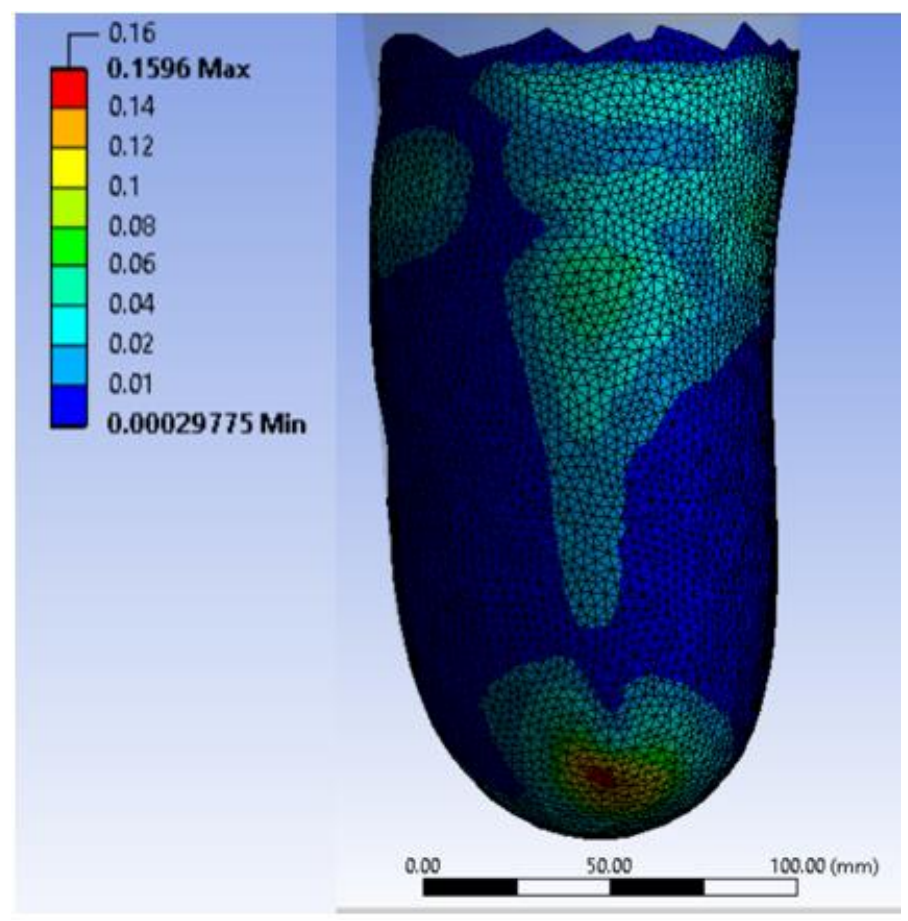

Figure 4.2 Limb Surface Stress for Limb Shape 2 [MPa]

The effectiveness of each liner/inlay type was judged based on its pressure gradient reduction capabilities relative to the "No Inlay" condition. A reduction in PPG is an indication of increased comfort for the patient and therefore an inlay configuration with a lower PPG is deemed a "better solution" compared to one with a larger PPG. It was necessary to ensure that the optimizations of the metamaterial conditions were directly targeting the reduction of the PPG as compared to the reduction of PPG simply being a byproduct of peak stress (PS) reduction. In order to check this, the PPG:PS ratio was calculated for each simulation. This ratio provides a direct comparison between how directly each liner/inlay reduces PPG compared to PS. For the applications of this research, a smaller ratio is desirable because it indicates that the PPG is being reduced at a greater rate compared to the PS. If the ratio is similar for all inlay configurations around a region 
of the limb, this indicates that the PS and PPG may be linearly correlated, which means the reduction of the PPG is not being directly targeted. It should be noted that this ratio is not a direct indication of the comfort achieved by the patient. The inlay that can achieve the greatest reduction in PPG should prove to be the most comfortable for the patient. The PS and PPG results are summarized for limb shape 1 (L1) in Table 4.1 and for limb shape 2 (L2) in Tables 4.2 and 4.3.

Table 4.1 Peak Stress and Peak Pressure Gradient Comparison at the Fibular Head [FH]

\begin{tabular}{|c|c|c|c|c|}
\hline & $\begin{array}{c}\text { Peak } \\
\text { Stress } \\
(\mathrm{PS}) \\
{[\mathrm{MPa}]}\end{array}$ & $\begin{array}{c}\text { Peak Pressure } \\
\text { Gradient } \\
(\mathrm{PPG}) \\
{[\mathrm{kPa} / \mathrm{mm}]} \\
\end{array}$ & $\begin{array}{c}\text { Gradient } \\
\text { Reduction } \\
\text { Compared to } \\
\text { "No Inlay" }\end{array}$ & PPG:PS \\
\hline No Inlay & 0.198 & 32.4 & - & 163.6 \\
\hline Silicone Liner & 0.134 & 22.0 & $32.1 \%$ & 164.2 \\
\hline Prosthetists Prescription & 0.175 & 22.1 & $31.5 \%$ & 126.3 \\
\hline Opt. Material Coefficients & 0.100 & 13.8 & $57.4 \%$ & 138.0 \\
\hline Opt. Material Layout & 0.165 & 21.7 & $33.0 \%$ & 131.5 \\
\hline $\begin{array}{l}\text { Opt. Material Coefficients and } \\
\text { Layout }\end{array}$ & 0.089 & 10.0 & $69.1 \%$ & 112.4 \\
\hline
\end{tabular}


Table 4.2 Peak Stress and Peak Pressure Gradient Comparison at the Tibial End

\begin{tabular}{|c|c|c|c|c|}
\hline & $\begin{array}{c}\text { Peak } \\
\text { Stress } \\
\text { (PS) } \\
{[\mathrm{MPa}]}\end{array}$ & $\begin{array}{c}\text { Peak Pressure } \\
\text { Gradient } \\
(\mathrm{PPG}) \\
{[\mathrm{kPa} / \mathrm{mm}]} \\
\end{array}$ & $\begin{array}{c}\text { Gradient } \\
\text { Reduction } \\
\text { Compared to } \\
\text { "No Inlay" }\end{array}$ & PPG:PS \\
\hline No Inlay & 0.160 & 16.9 & - & 105.6 \\
\hline Silicone Liner & 0.122 & 7.59 & $44.9 \%$ & 62.2 \\
\hline Prosthetists Prescription & 0.079 & 5.88 & $65.2 \%$ & 74.4 \\
\hline Opt. Material Coefficients & 0.069 & 5.16 & $69.5 \%$ & 74.8 \\
\hline Opt. Material Layout & 0.075 & 5.63 & $66.7 \%$ & 75.1 \\
\hline $\begin{array}{c}\text { Opt. Material Coefficients } \\
\text { and Layout }\end{array}$ & 0.069 & 4.46 & $73.6 \%$ & 64.6 \\
\hline
\end{tabular}

Table 4.3 Peak Stress and Peak Pressure Gradient Comparison at the Patellar Tendon

\begin{tabular}{ccccc}
\hline & $\begin{array}{c}\text { Peak } \\
\text { Stress } \\
(\mathrm{PS}) \\
{[\mathrm{MPa}]}\end{array}$ & $\begin{array}{c}\text { Peak Pressure } \\
\text { Gradient } \\
(\mathrm{PPG}) \\
{[\mathrm{kPa} / \mathrm{mm}]}\end{array}$ & $\begin{array}{c}\text { Gradient } \\
\text { Reduction } \\
\text { Compared to } \\
\text { "No Inlay" }\end{array}$ & PPG:PS \\
\hline No Inlay & 0.053 & 2.33 & $\mathbf{-}$ & 44.0 \\
Silicone Liner & 0.076 & 3.23 & $\mathbf{- 3 8 . 6 \%}$ & 42.5 \\
Prosthetists Prescription & 0.035 & 1.76 & $\mathbf{2 4 . 5 \%}$ & 50.3 \\
$\begin{array}{c}\text { Opt. Material Coefficients } \\
\text { Opt. Material Layout }\end{array}$ & 0.036 & 1.50 & $\mathbf{3 5 . 6 \%}$ & 41.7 \\
$\begin{array}{c}\text { Opt. Material Coefficients } \\
\text { and Layout }\end{array}$ & 0.037 & 1.56 & $\mathbf{3 3 . 0 \%}$ & 42.2 \\
\hline
\end{tabular}


Previous research shows a range of accepted peak limb stresses in both the simulation and clinical settings [10-11, 53-58,64]. When adjusted for the weight of the patient, the PS results of the "No Inlay" condition are within one standard deviation of the clinical results found by Yeung et al [64]. The clinical stress results found at the fibular head (FH), patellar tendon (PT), and tibial end (TE) were $1.82(.88), 1.95(1.49)$, and $1.51(1.27) \mathrm{kPa} / \mathrm{kg}$, respectively, validating the assumptions and approximations made in this model to mimic in vivo conditions.

The results show that the introduction of any type of soft interface material between the residual limb and hard prosthetic socket will almost always lead to a reduction in both the PS and PPG compared to an amputee that doesn't use any type of liner/inlay. The only exception is for the silicone liner at the patellar tendon (PT).

The metamaterial inlay, in all cases implemented, showed a greater reduction in PPG when compared to the homogenous silicone liner at all limb areas except for the prosthetist's prescription at the fibular head $(\mathrm{FH})$. This discrepancy, along with the "Optimized Material Layout" inlay at the FH were the only two examples of the PS being greater compared to the calculated value for the silicone liner. These results validate using the metamaterial as interface material between the residual limb and prosthetic socket to alleviate pressure build ups.

As expected, the "Optimized Material Coefficients and Layout" inlay proved to have the greatest PPG reduction at all regions on both limb models. The results also show that optimizing the material coefficients is a more effective method of reducing PPG as compared to just optimizing the material layout. 


\section{CHAPTER FIVE}

DISCUSSION

\subsection{Results Discussion}

While the assumptions and approximations used in the FEA produced results that were similar to those of clinical testing, they offer an explanation why the results presented differ from the published clinical results. One approximation is that the location of the areas of loading are not identical to in vivo reactions of the residual limb. Lin et al. [11] determined that so long as the load is applied evenly across the targeted bone surface, the effect of the location variance between FEA and clinical testing would be minimal.

One key assumption is that the soft tissue is treated uniformly around the entire limb, which does not account for potential differences between muscle, fat, skin, and scar tissues present in the limb. These differing stiffnesses could affect the current results. The two limb models represent non-traumatic amputations, and therefore, tissue distribution was assumed to be anatomically similar in the limb, which may not be the case in evaluations of other amputees. All assumptions and approximations were used to simplify the models in order to reduce computational time of the FEA calls, allowing the optimization results to be produced in a reasonable amount of time. Researchers take various approaches in determining the degree to which approximations and assumptions are used to simplify a model. So long as the approximations and assumptions produce results that can still be validated with previously determined clinical testing results, the researchers produce a viable method of mimicking the in vivo conditions of a transtibial amputee [5]. 


\subsection{Optimization Discussion}

This section will further discuss the relevance of the optimization results from Chapter 3 and how those results affect the design of the inlay. With regards to the material property optimization, the optimized material coefficients can be used to produce third order representations of the mechanical response. These third order representations can be compared to the third order representations of the metamaterials that underwent experimental compressive testing ( $\mathrm{DA}_{00}-\mathrm{DA}_{27}$ and Base material). This comparison shows how stiff or soft the materials are compared to the materials that had been tested. This comparison will give insight into what draft angles the optimized metamaterials should be $3 \mathrm{D}$ printed with in order to achieve the corresponding pressure reduction. The optimized layout section will discuss how the physical alignment of the materials change on the inlay compared to the prosthetist's prescription. The combined material property and layout optimization discussion will combine these two methods to show how the material should be designed and aligned around the inlay to achieve the largest reduction in peak pressure gradient.

\subsubsection{Material Property Optimization}

The purpose of optimizing the material properties and layouts of the metamaterial in the inlay is to show the increased pressure offloading (e.g. PPG reduction) potential that the metamaterial could provide when utilized as an interface material between the residual limb and a hard-prosthetic socket. To better understand the physical meaning of the optimized material coefficients found from the material property optimization, the third order stress-strain curves were produced by plugging the optimized material coefficients 
into Eq 2.1 and compared to the stress-strain curves of the current third order representations of the metamaterial $\left(\mathrm{DA}_{00}-\mathrm{DA}_{27}\right)$. These stress-strain curves allow for a visual comparison between the optimized material properties and how they behave relative to the third order representations of the metamaterials with predetermined draft angles.

Figure 5.1 was created by plugging the optimized Yeoh coefficients for the fibular head $(\mathrm{FH})$ found in Table 3.1 into Eq 2.1. These stress-strain curves show how the optimized material properties around the $\mathrm{FH}$ compare to the current metamaterial variations.

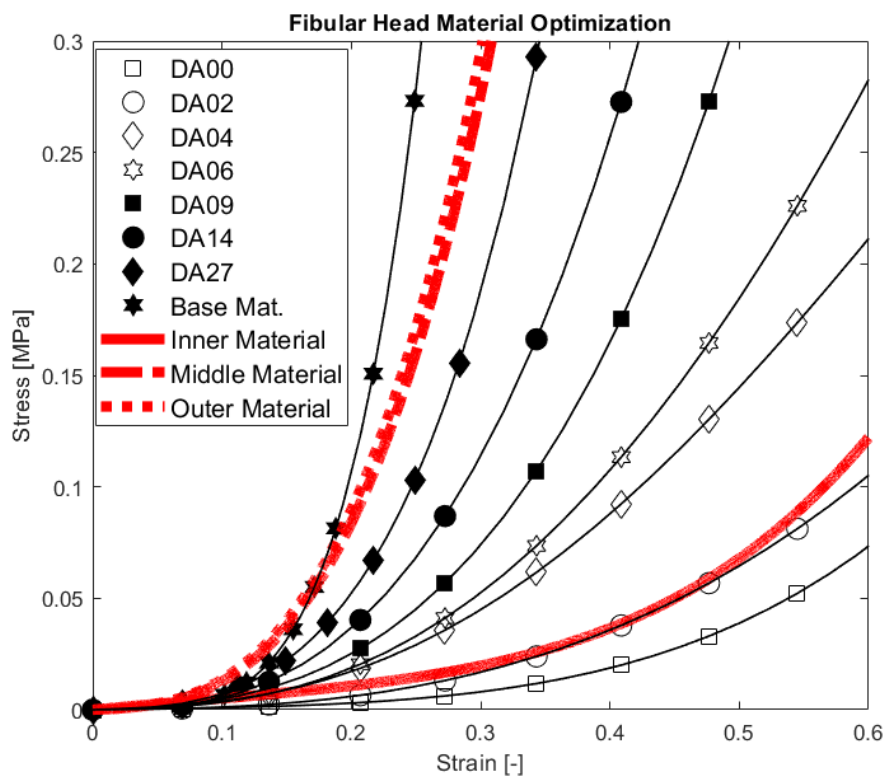

\section{Figure 5.1 Stress-Strain Comparison Between "Optimized Material Properties" and Current Material Properties at the Fibular Head (FH)}

Figure 5.1 shows that the inner most material around the FH should be offloaded with a material much softer than the prosthetist's original prescription of $\mathrm{DA}_{09}$. In fact, the inner material shows properties like those of $\mathrm{DA}_{02}$. The middle and outer material show near 
identical results. This may indicate that the pressure offloading at the FH could be achieved using only two materials, or that limiting the offloading to just three materials is not sufficient. Both middle and outer materials have a response somewhere between that of $\mathrm{DA}_{27}$ and the base material. This suggests the need to launch a further investigation into increasing the draft angle variations available for the metamaterial. Figure 5.1 shows that a draft angle larger than $27^{\circ}$ is necessary in order to more effectively offload the FH. The stiffest optimized material was closer to the prosthetist's prescription of $\mathrm{DA}_{27}$ as compared to his prescription of $\mathrm{DA}_{09}$ and the softest optimized material. These results produce an inlay that satisfies the recommendation by Sanders et al. [29] to have a soft material offload the boney prominences and a stiffer material provide support to the soft tissue.

Figure 5.2 was created by plugging the optimized Yeoh coefficients for the tibial end (TE) and patellar tendon (PT) from Table 3.2 into Eq 2.1. These stress-strain curves show how the optimized material properties around the TE and PT compare to the current metamaterial variations. 

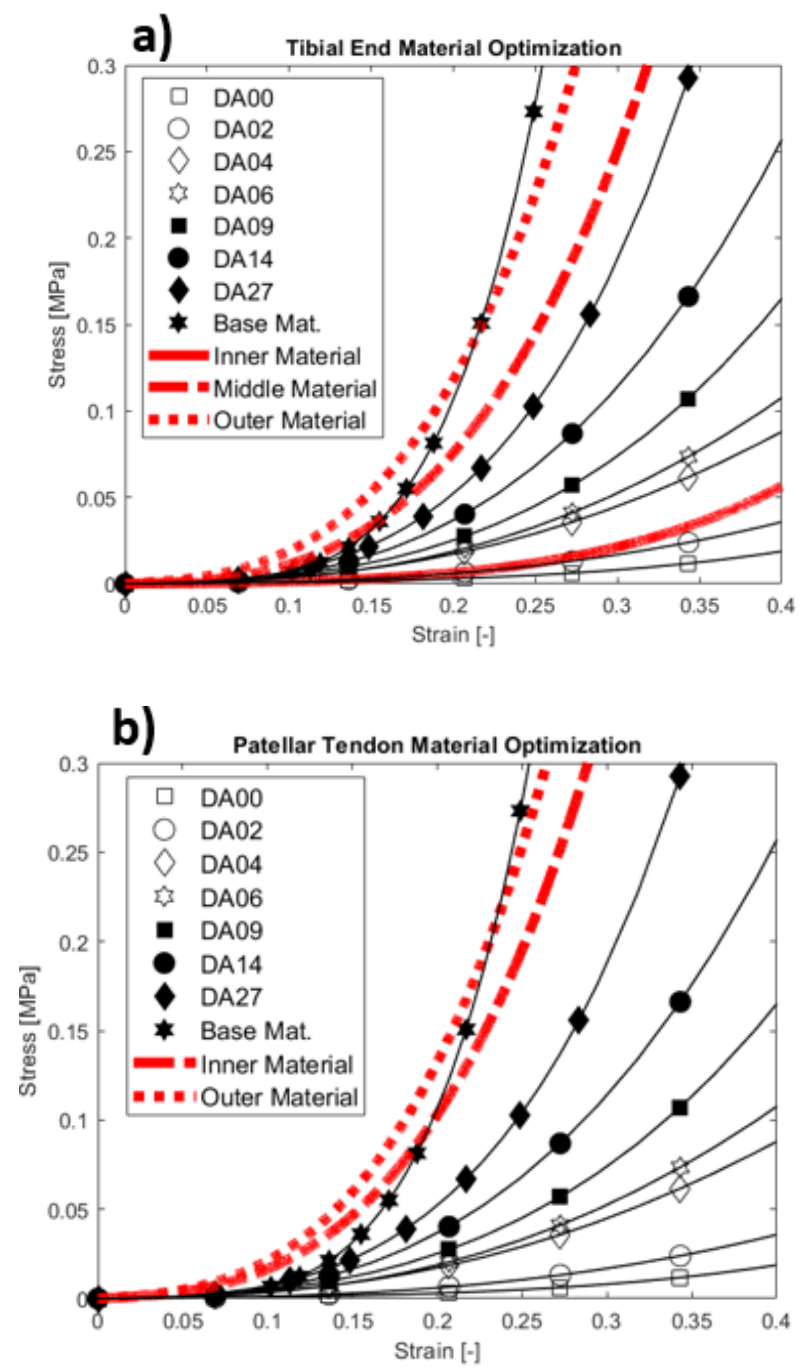

\section{Figure 5.2 Stress-Strain Curve Comparison Between "Optimized Material Properties" and Current Material Properties for a) Tibial End (TE) b) Patellar Tendon (PT)}

With regards to the tibial end (TE), Figure 5.2A shows that the softest material should be modeled more like $\mathrm{DA}_{02}$ as compared to the prosthetist's recommendation of $\mathrm{DA}_{09}$. This recommended material property is unexpected at the TE. Due to the combined normal and shear forces, it was expected that a stiffer material than $\mathrm{DA}_{02}$ would be the softest material to offload the TE. A stiffer material would be able to reduce the shear stress that is subjected 
to the residual limb [29]. If a part of the limb is only subjected to normal stress, like the fibular head $(\mathrm{FH})$, then a very soft material like $\mathrm{DA}_{02}$ would be expected. The middle and outer materials are stiffer than the prosthetist's recommendation. Unlike the FH, the middle and outer materials of the TE are distinct enough to warrant a three-material design. Both materials fall within $\mathrm{DA}_{27}$ and the base material, which furthers the claim that the list of available draft angles should be increased.

With regards to the patellar tendon (PT), Figure 5.2B shows that both materials should be stiffer than the prosthetist's prescription of $\mathrm{DA}_{14}$ and $\mathrm{DA}_{27}$ as the middle and outer materials, respectively. The inner and outer materials show similar results but are still distinct enough to warrant two materials for offloading. The outer material should be like the base material while the inner material should have a draft angle larger than $\mathrm{DA}_{27}$. These results show that the PT does not require materials as soft as those for the TE or FH to alleviate pressure. These results make sense because the PT is subjected to a greater magnitude of shear force as compared to normal force. A stiffer material on the inlay ensures that the residual limb does not have to endure most of the shear stress that arises from this shear force. A softer material would subject the residual limb to a greater magnitude of shear stress and therefore a larger equivalent stress [29]. This explains why the peak stress (PS) and peak pressure gradient (PPG) increased at the PT when the silicone liner is used. A stiffer material ensures that the shear stress is limited on the residual limb which leads to a more comfortable experience for the patient. This claim is backed up by the shear stress comparison between the silicone liner and optimized material properties shown in Figure 5.3 which shows that the shear stress is drastically reduced with the stiffer 
optimized materials at the PT. It should be noted that the stress ranges of Figure 5.3 cover the entire limb surface and not just the patellar tendon, explaining why the maximum shear stress at the patellar tendon was specifically called out.
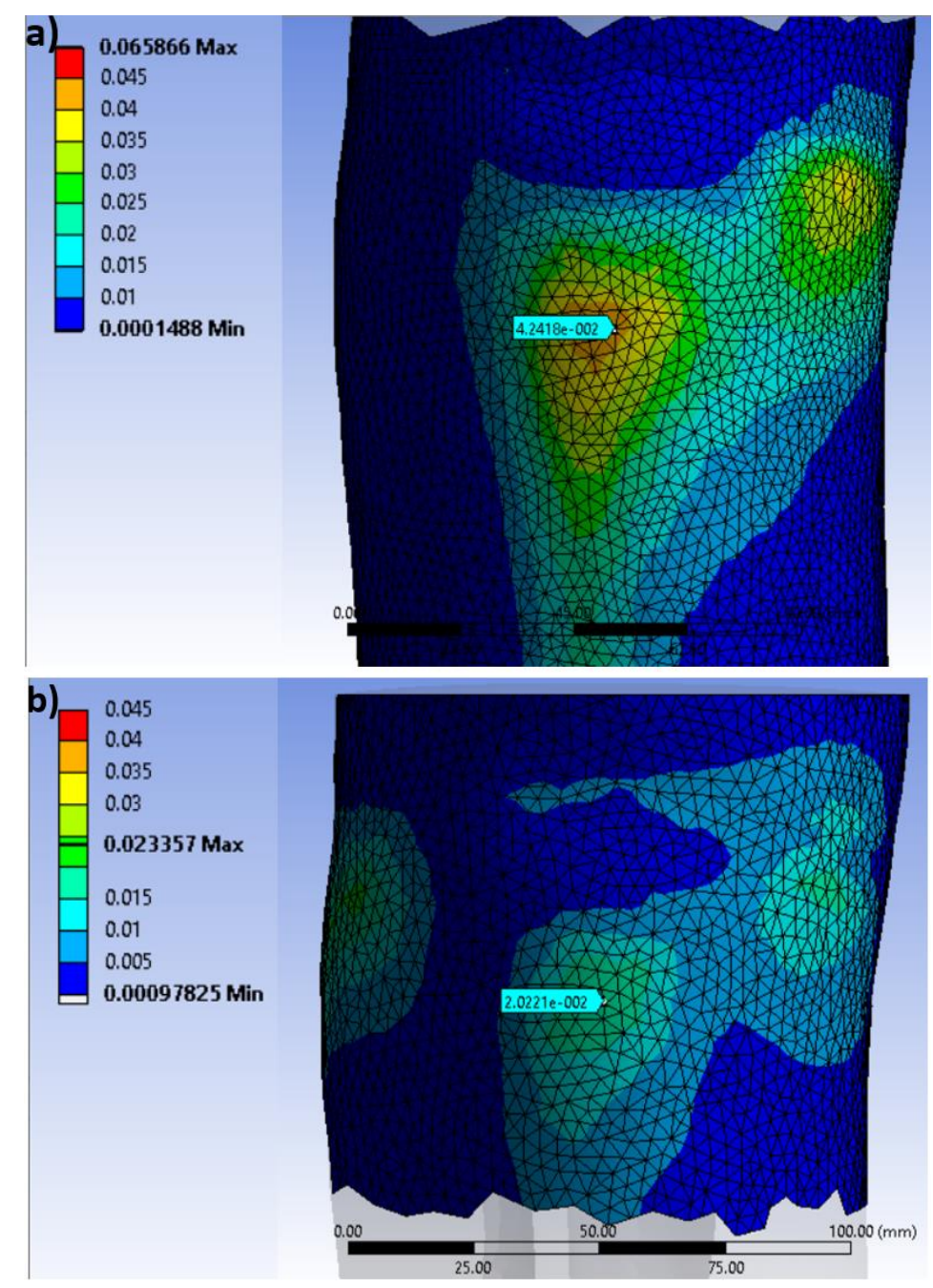

Figure 5.3. Limb Surface Shear Stress a) Silicone Liner b) Optimized Material Properties

\subsubsection{Material Layout Optimization}

Table 4.1-4.3 shows that optimizing the material layout has a less drastic effect on reducing the peak pressure gradient (PPG) as compared to optimizing the material properties. Optimizing the layout of the prosthetist's prescribed material properties 
achieves a greater reduction in PPG than the prosthetist's prescribed inlay. Figure 5.4 shows the dimensional changes between the optimized layout inlay and prosthetist prescribed inlay at the fibular head $(\mathrm{FH})$.
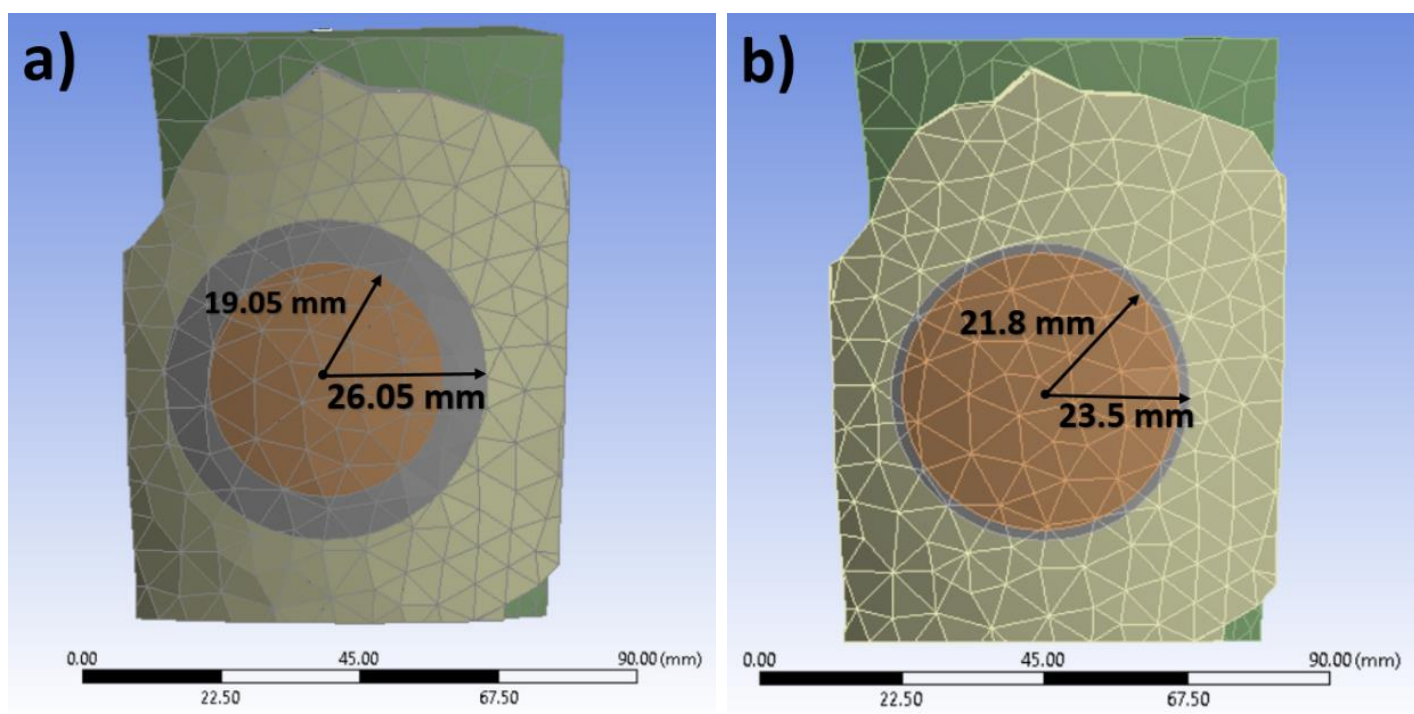

\section{Figure 5.4 Fibular Head Inlay Material Layout Dimensions a) Prosthetist's Prescription b) Optimized Layout}

The optimized material layout of the inner material (Orange region) is very similar to the prosthetist's prescription. The outer region of $\mathrm{DA}_{27}$ (Beige region) also has a similar layout to the prosthetist's prescription. The biggest difference is with regards to the middle material (Grey region). The middle material is limited to a small circular region with a thickness less than $2 \mathrm{~mm}$. This means that the middle region of $\mathrm{DA}_{14}$ is doing very little to aid in the offloading of pressure. This may be further evidence that the FH can be offloaded using just two materials.

Figure 5.5 shows the inlay dimensional changes at the patellar tendon (PT) for L2. 

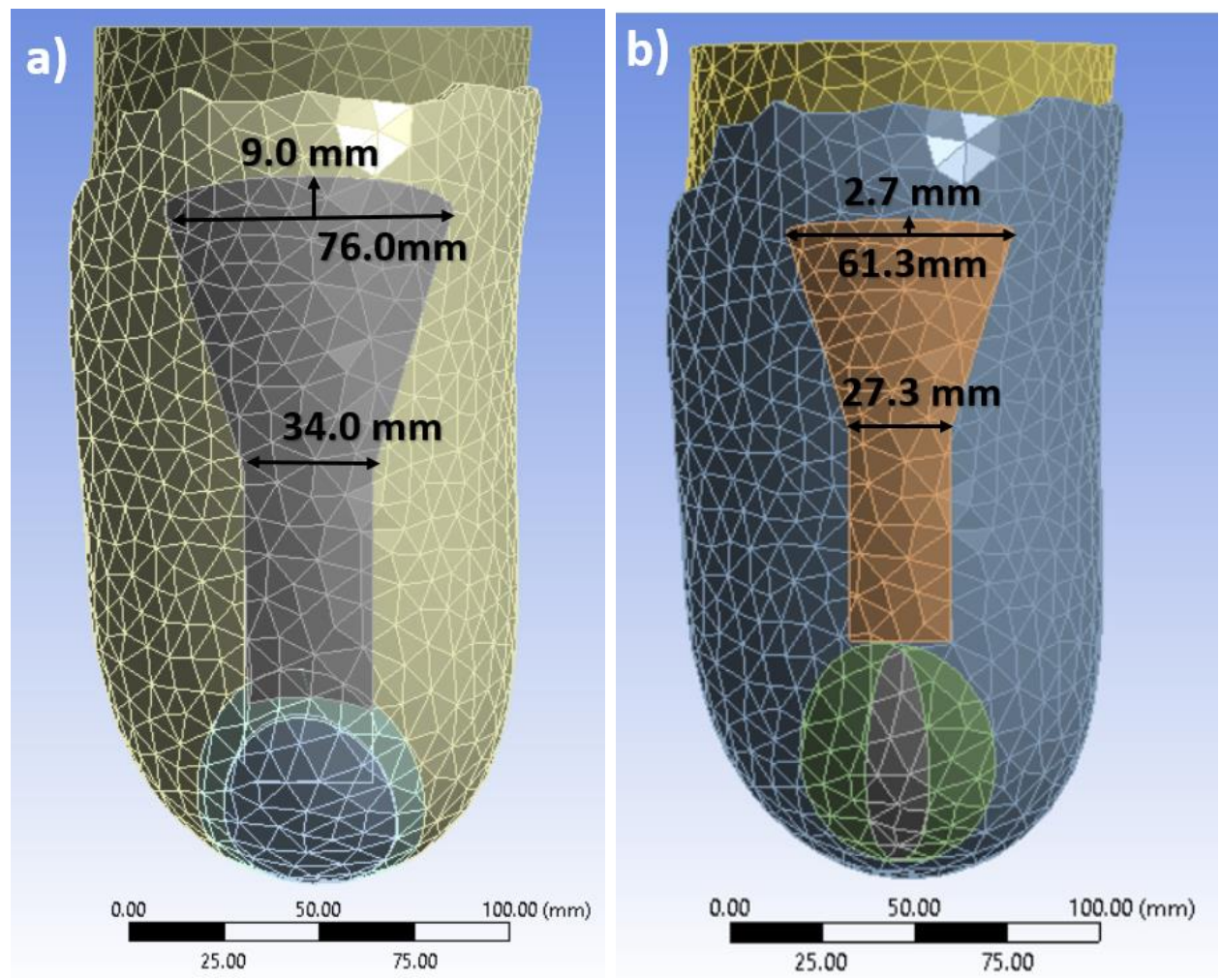

\section{Figure 5.5 Patellar Tendon Inlay Material Layout Dimensions a) Prosthetist's Prescription b) Optimized Layout}

Figure 5.5 shows that the optimized layout reduces all the key components slightly compared to the prosthetist's prescription. These reductions isolate the region of $\mathrm{DA}_{14}$ (5.5A Grey and 5.5B Orange regions) closer around the area of high stress. This ensures that the slightly less stiff material is limited to just the necessary regions to allow for the stiffer $\mathrm{DA}_{27}$ (5.5A Beige and 5.5B Blue regions) to provide the increases stability at the PT. This logic matches with the results of the optimized material properties saying that a stiffer material is more beneficial for pressure offloading at the PT. By isolating the $\mathrm{DA}_{14}$ region closer to the boney prominences, the $\mathrm{DA}_{27}$ is given a larger area to provide the stability that reduces the shear stress on the residual limb. 
Figure 5.6 shows that the dimension changes at the tibial end (TE) for L2.
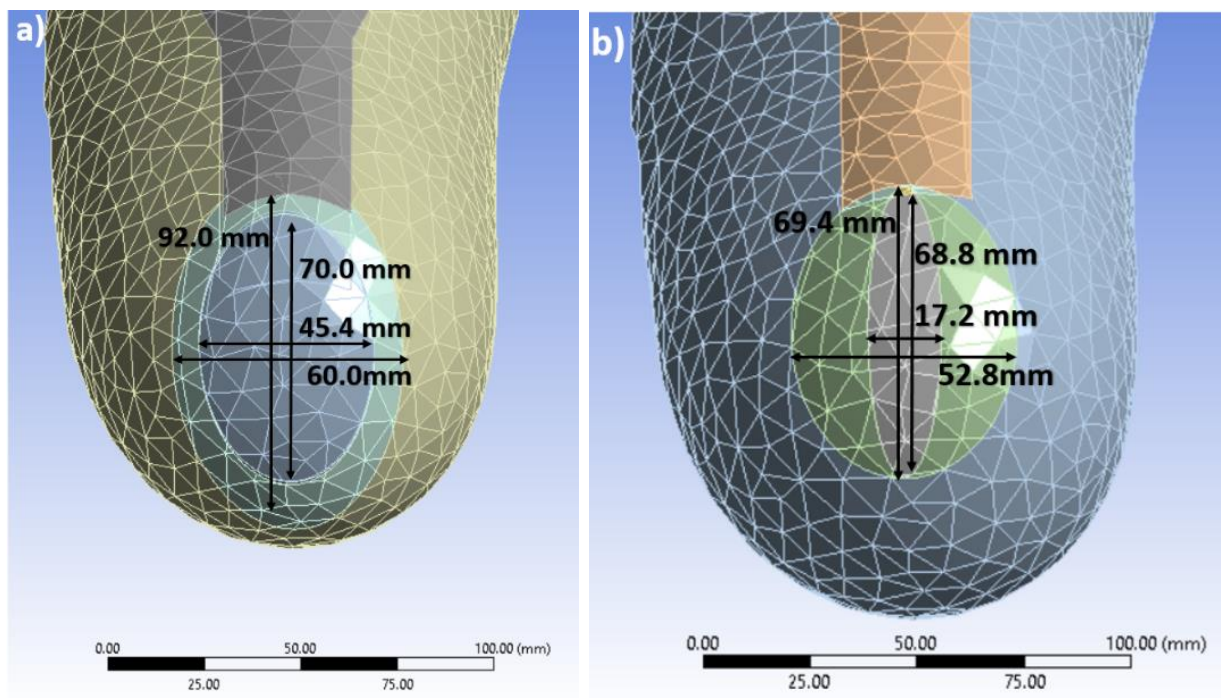

\section{Figure 5.6 Tibial End (TE) Inlay Material Layout Dimensions a) Prosthetist's Prescription b) Optimized Layout}

Figure 5.6 shows that the optimized layout for the TE also reduces all the dimensions of the prosthetist's prescription. The most noticeable change is the internal ellipse width (W3 from Figure 3.10) reducing from $45.4 \mathrm{~mm}$ to $17.2 \mathrm{~mm}$. The reduction ensures that the soft $\mathrm{DA}_{09}$ (5.6A Blue and 5.6B Grey Regions) is isolated around the area of high stress. Figure 5.6 also shows that the dual ellipse material layout the prosthetist recommended may not be the best layout. The difference in sizes between the ellipse heights ( $\mathrm{H} 2$ and $\mathrm{H} 3$ ) is significantly smaller than the difference between the ellipse widths (W3 and W4). There is a very limited region of $\mathrm{DA}_{14}$ (5.6A Aqua and 5.6B Green Regions) in the vertical direction between $\mathrm{H} 2$ and $\mathrm{H} 3$, while there is a noticeable area of $\mathrm{DA}_{14}$ in the horizontal direction between W3 and W4. This may imply that offloading at the TE can be better achieved using a thin internal ellipse with two mirrored crescent moon shapes on either side of it. 


\subsubsection{Material Property and Layout Optimization}

The material property and layout optimization increased the number of design variables to allow increased customization into the design of the metamaterial inlay to meet the specific needs of the patient. As Tables 4.1-4.3 show, optimizing the layout and properties simultaneously leads to the greatest reduction in the peak pressure gradient (PPG) of the methods available. These results are to be expected because this simultaneous optimization allows for the inlay to be designed to offload the areas of high stress around the boney prominences with a soft material and provide stability to the areas of soft tissue with a stiffer material.

Figure 5.7 shows the comparison between all three material layouts at the fibular head (FH) for limb shape 1 (L1). Figure 5.8 shows the stress-strain comparison of the material properties from the material property and layout optimization at the $\mathrm{FH}$. 

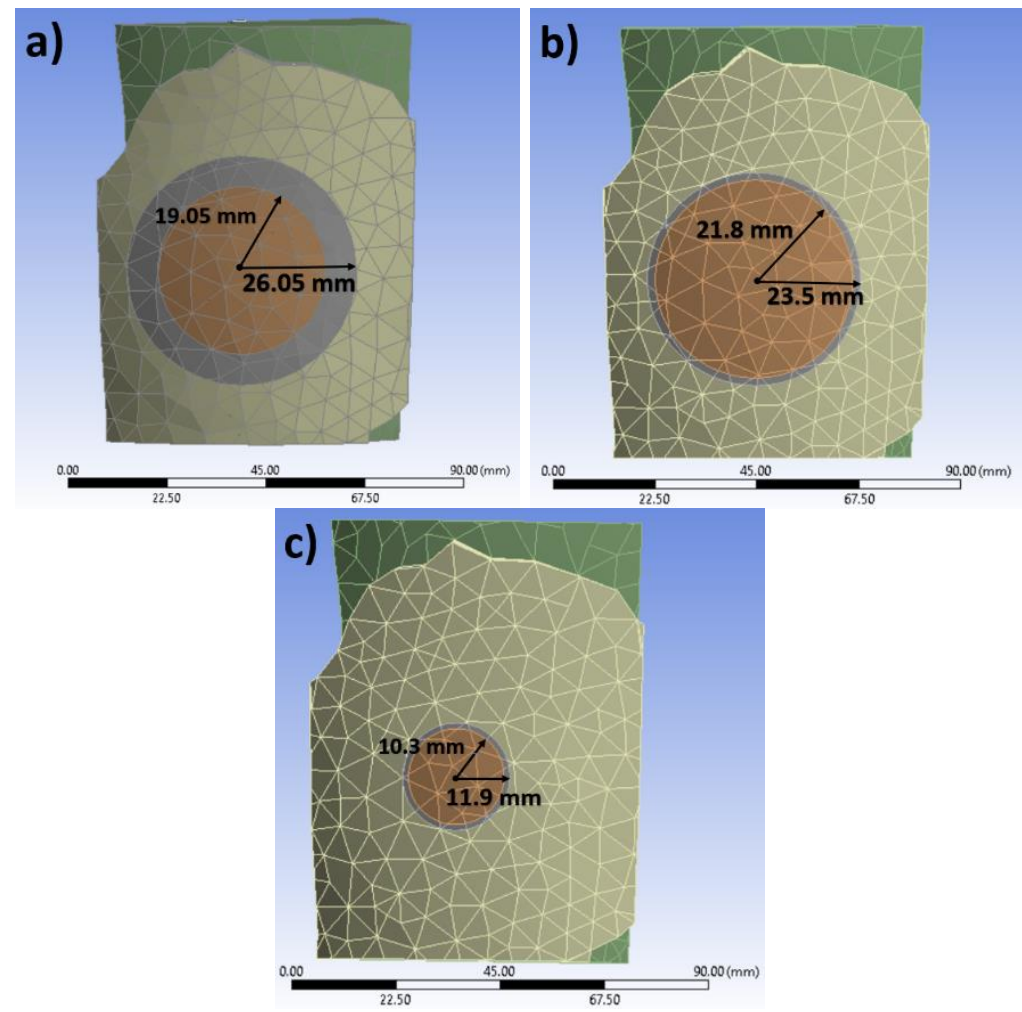

Figure 5.7 Fibular Head Inlay Material Layout Dimensions Full Comparison a) Prosthetist's Prescription b) Optimized Layout c) Optimized Layout w/ Optimized Material Properties

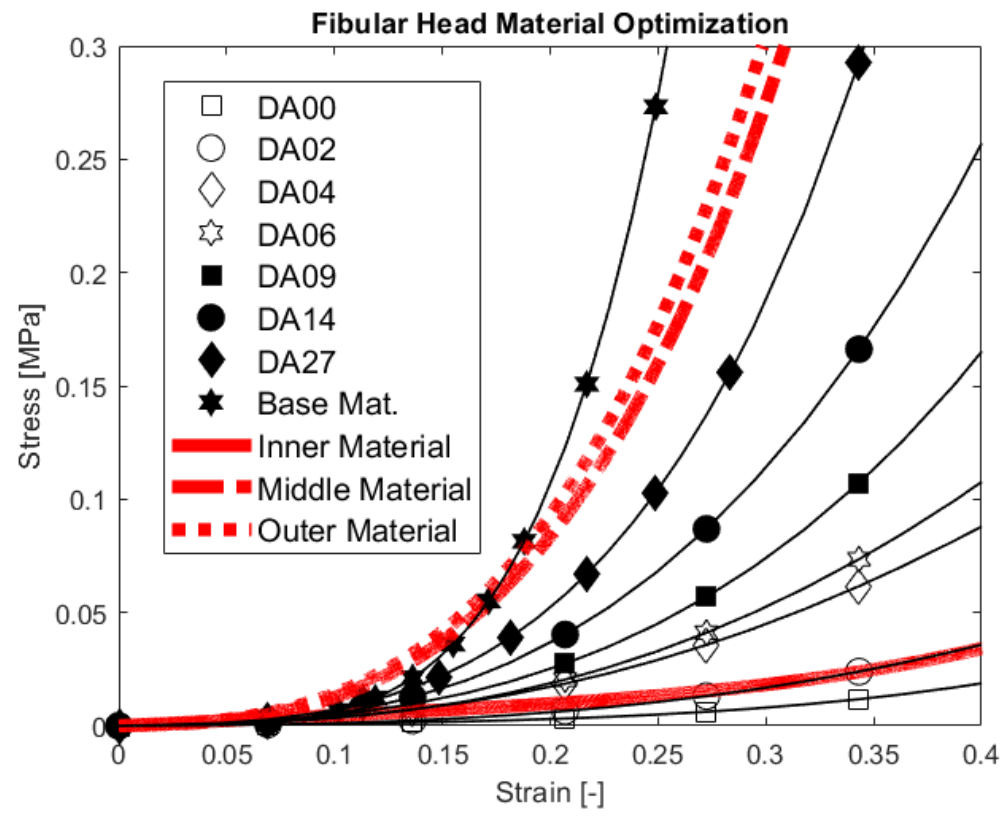

Figure 5.8 Stress- Strain Comparison for Fibular Head Material Property Optimization with Material Layout Optimization 
Figure 5.7 shows that the area of the softest material has been drastically reduced to be isolated around the FH. Figure 5.8 shows that this soft internal material should behave almost identically to $\mathrm{DA}_{02}$, confirming the results of Figure 5.1 that the inner material should be softer than the initially prescribed $\mathrm{DA}_{09}$. The very soft material being isolated to a small area makes sense because it ensures that the highly compliant material is being isolated to the area of highest pressure. The soft material will deform more than a stiffer material, therefore providing a larger area for the forces to be applied to, which in turn reduces the overall pressure [12]. The middle and outer materials continue to show very similar material properties. These materials are slightly softer than prescribed in the material property optimized, but they are still stiffer than the original prescription of $\mathrm{DA}_{27}$. Figure 5.7 shows that the optimized layout still has a very small region of the middle material. This is the third indication, along with the middle and outer materials having similar material properties, that the FH can be properly offloaded using just two materials. The take-away from these results is that the FH should be offloaded using a very soft material that is isolated just around the FH protrusion on the residual limb. Outside of that small region, a stiff material should be applied to provide the necessary stability to the limb while still aiding in the reduction in PPG.

Figure 5.9 shows the comparison for all three inlay layouts at the patellar tendon (PT) for limb shape 2 (L2). Figure 5.10 shows the stress-strain comparison of the material properties from the material property and layout optimization at the PT. 

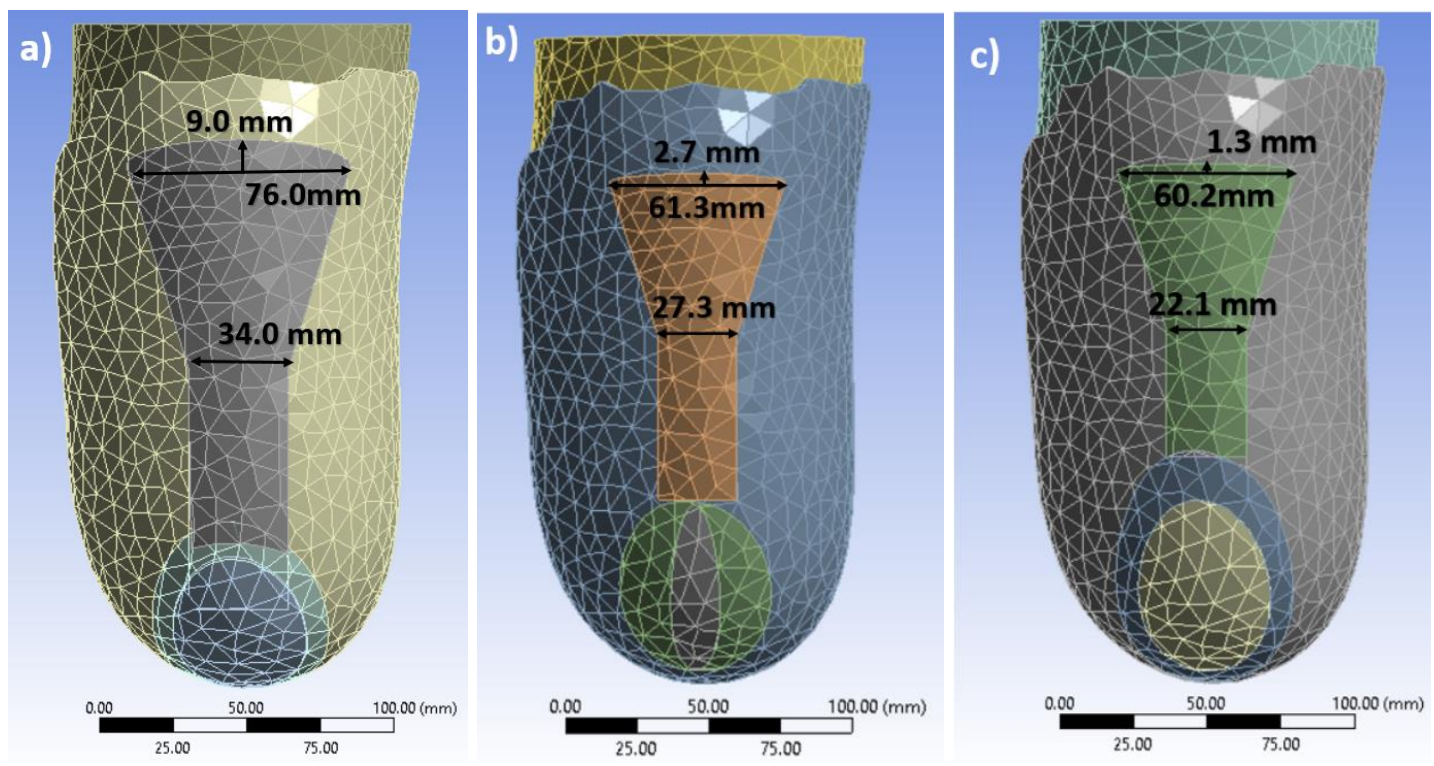

Figure 5.9 Patellar Tendon Inlay Material Layout Dimensions Full Comparison a) Prosthetist's Prescription b) Optimized Layout c) Optimized Layout w/ Optimized Material Properties

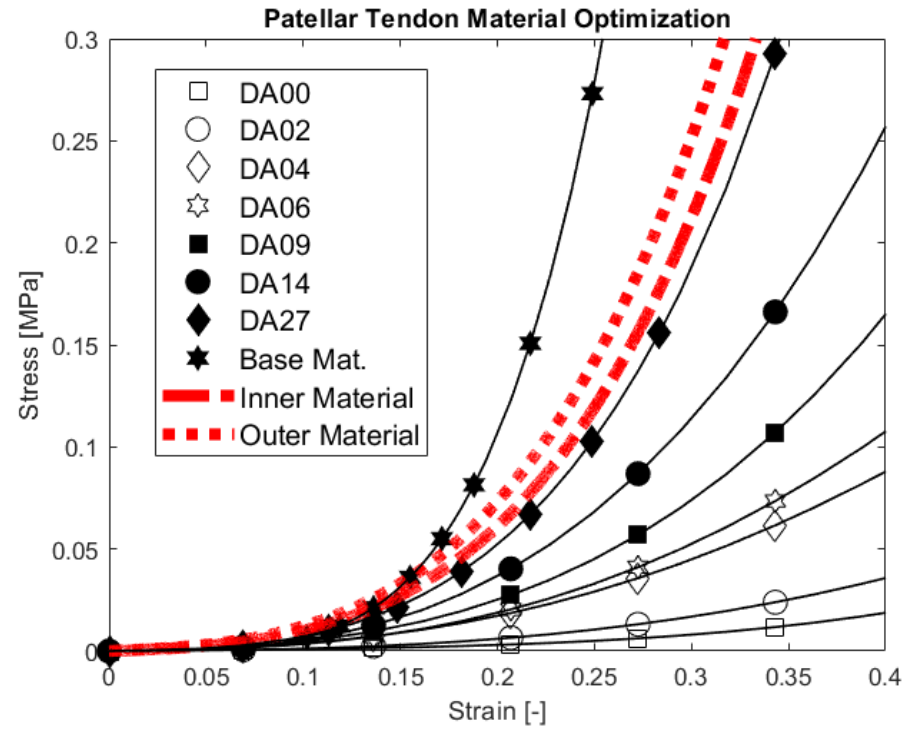

Figure 5.10 Stress-Strain Comparison for Patellar Tendon Material Property Optimization with Material Layout Optimization

Figure 5.9 shows that the material layout does not change significantly compared to the prosthetist's prescription or the optimized layout results. All the dimensions have been further reduced to continue to isolate around the area of highest stress. Figure 5.10 shows 
that the optimized material properties should be slightly stiffer than $\mathrm{DA}_{27}$. These materials are much stiffer than the soft materials that are recommended to offload the FH for L1, again backing the claim that the PT requires stiffer materials in order to reduce the amount of shear stress that is applied to the residual limb from the large magnitude of shear force the PT experiences. The difference between the inner and outer materials at the PT are minimal. It would require patient input to determine if a single or double material prescription is more comfortable. Both materials should be stiffer than $\mathrm{DA}_{27}$, but softer than the base material, further prompting the need to launch an investigation into a wider range of draft angles to further explore the region between $\mathrm{DA}_{27}$ and the base material.

Figure 5.11 shows the comparison for all three inlay layouts at the tibial end (TE) for limb shape 2 (L2). Figure 5.12 shows the stress-strain comparison of the material properties from the material property and layout optimization at the TE.
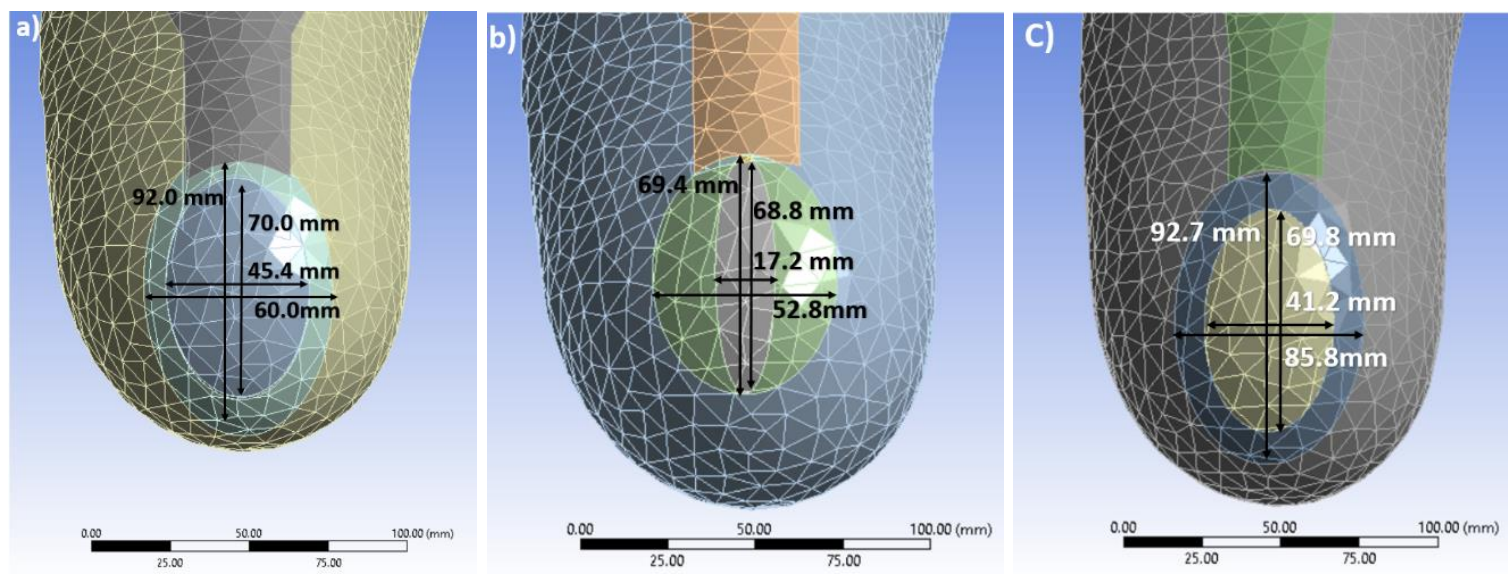

Figure 5.11 Tibial End Inlay Material Layout Dimensions Full Comparison a) Prosthetist's Prescription b) Optimized Layout c) Optimized Layout w/ Optimized Material Properties 


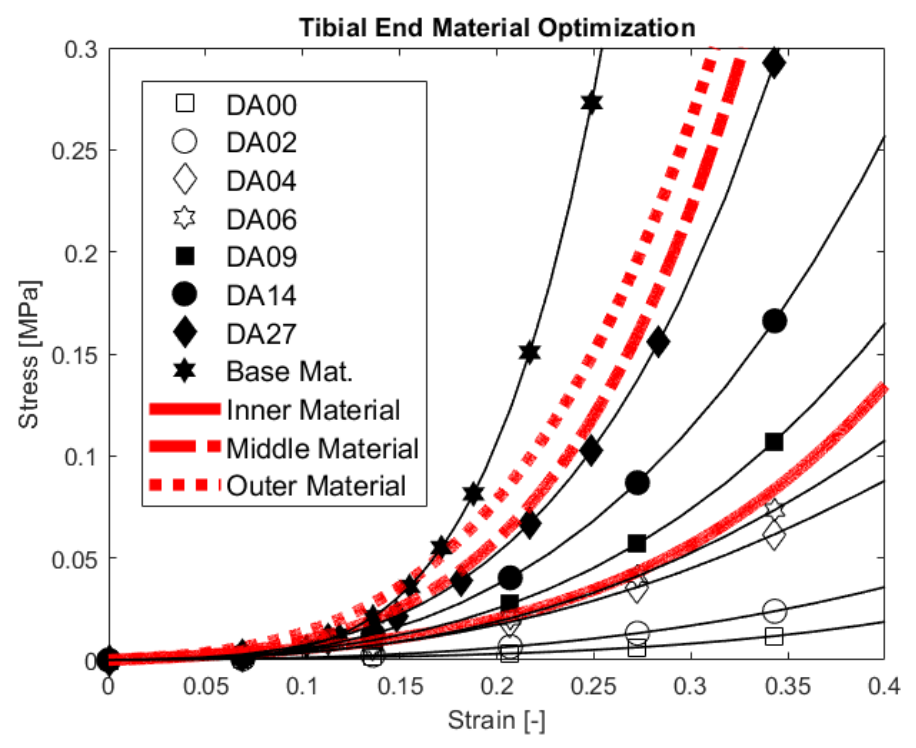

\section{Figure 5.12 Stress-Strain Comparison for Tibial End Material Property Optimization with Material Layout Optimization}

Figure 5.11 shows that the inlay layout returns to a similar design as the prosthetist's prescription. The soft inner material, which Figure 5.12 shows should be between $\mathrm{DA}_{06}$ and $\mathrm{DA}_{09}$, has a much larger area than the optimized layout inner material. The softest material of Figure 5.11 is much stiffer than the softest material of Figure 5.2 when just the material properties were optimized. This difference is caused by the introduction of a shear force. Unlike the patellar tendon (PT), most of the load applied to the TE is normal force caused by compression, but the anterior-posterior shear introduces a shear force on the TE. The combination of the normal and shear forces means that the materials must be soft enough to allow proper offloading of the normal stress around the boney prominences, but also have the rigidity to provide the stability to offload the shear stress, validating the optimized material being slightly softer than $\mathrm{DA}_{09}$. The middle and outer materials should have draft angles somewhere between $\mathrm{DA}_{27}$ and the base material, once again, prompting 
the need to increase the available draft angles. The middle region does have a distinct thickness that warrants the need to offload the TE using three materials. Based on the results of Figure 5.11 and 5.12, the prosthetist's prescription was closest to the optimized material properties and layout at the TE.

\subsection{Clinical Relevance Discussion}

A key factor to consider in this research is how a patient will react to the metamaterial inlay. Patient comfort is highly subjective for each patient [16-18]. An inlay could be designed to minimize the peak stress and peak pressure gradient, but if the patient does not feel comfortable then the solution is unviable. One area of potential concern could be the drastic change in mechanical response between two materials. This change could be felt as a rigid point on the inlay, potentially explaining the prosthetist prescribing a three-material solution for the fibular head (FH) and tibial end (TE), for the respective limb shapes. The prosthetist anticipated that the mechanical response change between $\mathrm{DA}_{09}$ and $\mathrm{DA}_{14}$ and $\mathrm{DA}_{14}$ to $\mathrm{DA}_{27}$ is gradual enough to avoid a negative reaction from the patient. Care must be taken when applying the optimized material property results in a clinical setting to ensure that the mechanical response changes between the metamaterials are not too severe to warrant a patient complaint. The $\mathrm{FH}$ and $\mathrm{TE}$ results are relevant examples. The optimized material property results show a clear distinction between the softest material and the other two materials. While these material selections will minimize the PPG, the patient must be consulted to ensure that the metamaterial differences are not too severe to cause discomfort. 
Another area of concern is selecting a metamaterial configuration that a patient considers too soft. Experience has shown that patients can label inlay materials that are too soft as "squishy". This negative reaction will affect a patient's confidence that the inlay will be able to hold up to the rigors required to provide comfort and stability to the amputee. Unfortunately, an acceptable material stiffness range will be highly subjective for each patient. Therefore, patient input would be necessary to determine if a selected metamaterial is too soft. A method to reduce the chances of the patient finding the selected metamaterial too soft is to add a constraint on the maximum deflection of the inlay to the optimization problem. This added constraint will ensure that the inlay has material properties that can provide enough rigidity to instill confidence into the amputee.

While these potential patient concerns need to be addressed, this research has shown the prospective benefits of the metamaterial inlay. The methods investigated have opened the door to the possibility of providing a more customized interface material solution to transtibial amputees. This research can be used to aid in the prescription process to help prosthetists find a more comfort solution for amputees in a shorter time. The current practice patterns predict between 15-45 visits with a prosthetist for a lower-limb amputee to receive the appropriate care to feel confident in using the prosthesis daily. Many of the initial visits involve fitting the amputee with a socket and liner and making the necessary adjustments for the amputee to be comfortable. It is incredibly rare for the prosthetist to design the proper socket shape and select the proper liner material in the first visit [65]. The prosthetist attempts to prescribe a solution for a patient based on previous experience. Due to the variability of individual patients, this method can lead to an excess of visits and 
failed socket models. Each additional visit and failed socket model are wasted time and money. Utilizing an optimized metamaterial inlay that has been designed to meet the unique loading conditions and limb shapes of an amputee should drastically reduce the time it takes to achieve comfort for the amputee. The faster an amputee feels comfortable regularly wearing the prosthesis, the faster the amputee can return to living a more normal life. 


\section{CHAPTER SIX \\ FUTURE WORK AND CONCLUSION}

This research has begun to investigate the potential of utilizing metamaterials as an interface material between the residual limb and prosthetic socket of transtibial amputees. The research presented has shown that a single layer metamaterial can be implemented as an interface material to offload pressure in a transtibial prosthesis. Looking back at the research objectives of this thesis:

1. Determine the hyperelastic material properties of the selected metamaterial.

Using uniaxial compression testing, the metamaterials showed hyperelastic material properties which satisfied the desire to find an inlay material that behaved similarly to soft tissue. The metamaterial variations with the smaller draft angles showed clear buckling under compression due to the reduced wall thickness between unit cells.

2. Determine a method to provide a simple representation of the material properties.

The hyperelastic material properties of this single layer metamaterial can be represented using a Yeoh $3^{\text {rd }}$ order material model. The Yeoh $3^{\text {rd }}$ order model uses three customizable material coefficients to define the mechanical response of the material. This material model gave an accurate third order representation of the mechanical response of the metamaterial and provided a method for the material properties to be optimized.

3. Determine the reduction in peak pressure gradient (PPG) on the residual limb with an inlay utilizing the metamaterial that has been designed by a prosthetist.

The metamaterial was shown to reduce peak stress and peak pressure gradients to a greater extent than a common silicone liner around three key locations of the residual limb, suggesting that heterogenous material property liners are better able to increase comfort 
for amputees through alleviation of surface limb pressures. These results also validated the potential to implement the selected metamaterial as an interface material between the residual limb and prosthetic socket.

4. Optimize the design of the inlay to achieve a further reduction in PPG.

The material properties can be optimized to meet the unique performance needs of an amputee. The resulting optimized coefficients offer insight into how the metamaterial geometric parameters should be designed for an individual patient. The layout of this metamaterial within an inlay can also be optimized to better adapt to the unique limb shapes of amputees. Combining the optimization of the material properties and layouts provides a prosthetist with a method to prescribe a more customizable inlay that reduces peak pressure gradients. It was shown that utilizing at least one form of inlay design optimization, whether that be material property, material layout, or both, leads to a greater reduction in pressure gradients compared to an inlay designed by a prosthetist's intuition and previous experience alone. Optimizing the material properties proved to be more effective at reduction PPG compared to optimizing the material layout alone. Optimizing the material properties and layout simultaneously lead to the greatest reduction in PPG at all tested locations around the residual limb. The implementation of inlay design optimization can aid the prosthetist during the prescription process of an amputee's treatment. This research has validated that the selected metamaterial has the potential to meet the unique offloading needs of an individual patient, leading to an increase in comfort and functionality.

In coordination with the research presented, a clinical trial is in progress to determine the in vivo pressure offloading capabilities of the metamaterial. The six-patient trial is 
comparing the residual limb pressure mapping data when the patients wear a prosthetist's designed metamaterial inlay and when the patients use their traditional prosthetic liner. The study allows for a month-long acclimation period for each patient to become normalized with wearing the metamaterial inlay. Following the month, the patients will be asked for their opinions of the inlay, including comfort, mobility, and appearance. A goal of this study is to validate the FEA results as well as determine if the metamaterial is deemed a more useful interface material for transtibial amputees.

There are still several avenues pertaining to metamaterial applications with transtibial amputees that should be investigated. One expansion of this research that is currently being discussed is the creation of isolated offloading pads that can be implemented around areas of the residual limb that require additional offloading. These pads would utilize the metamaterial but instead of having the large sizes of the inlays in this research, they would be small enough to just target one area of the limb. It would be a similar concept as what was done to isolate the model around the fibular head for limb shape 1. These offloading pads could be designed in two ways.

The first would be to come up with a predetermined "catalog" of options. These options would be created by running a series of material property and layout optimizations for the metamaterial on a range of different limb models using a variety of loading situations. The high number of optimizations would allow for a series of offloading pads to be designed to meet the needs of a certain limb shape and loading conditions. The offloading pads would have unique shapes to offload the fibular head, tibial end, tibial crest, or patellar tendon. A patient would come in and based on that patient's limb shape, 
loading conditions, weight, activity level, etc., the patient would be prescribed one of the predetermined offloading pad designs. This method groups a large number of patients into specific groups that require a similar style of offloading. While this method does not provide complete customization, it allows for a quick and inexpensive, yet effective, offloading solution for a high number of patients. The biggest concern would be if a patient does not fall into one of the predetermined groups, then this method would not be viable.

The other design method would be similar to what was done in this research. A patient would visit a prosthetist and the patients limb model would be scanned and the loading conditions and activity level determined. From there, a material property and layout optimization would be run utilizing an FEA set up from the patient's information. The offloading pad would be designed in accordance with the optimization results. This method would take longer and be more expensive, but it provides a more customizable solution that specifically target the pressure offloading needs of an individual patient.

The biggest limitation of this research is the metamaterial only targeted pressure offloading. There is no attempt to alleviate heat and moisture concerns, which are common complaints among transtibial amputees [22-24]. An investigation should be done to look at a way of producing a metamaterial that can be adjusted to meet certain pressure offloading concerns as well as heat and moisture dissipation requirements. There has not be an effort by this research team to investigate how a metamaterial might be able to satisfy all these criteria. It is expected that there would be a trade-off between pressure offloading and heat/moisture dissipation. This trade-off could be controlled my altering a series of physical design variables, similar to altering the draft angle in the metamaterial discussed 
in this research. A prosthetist could use patient input to determine how the metamaterial should be designed to meet the unique offloading, heat, and moisture concerns to achieve the greatest level of comfort.

Continued development towards the metamaterial discussed in this thesis could include creating a better material model that is able to capture the buckling seen in the lower draft angle materials. Another avenue would look at the metamaterials application towards other prosthetics including transfemoral or foot amputations. Finally, embedding sensors could be used to track in vivo stresses of a patient's residual limb to further validate FEA results. All of these avenues of future research will continue to push the development of this and other metamaterials to provide increased comfort and mobility to transtibial amputees. 


\section{APPENDICES}

\section{APPENDIX A. Relevant MATLAB Code}

Relevant MATLAB Code can be found on Nathan Brown's Github account:

https://github.com/nkbrown503/MastersThesis2020/tree/master

\section{APPENDIX B. Limb Surface Stress Mappings}
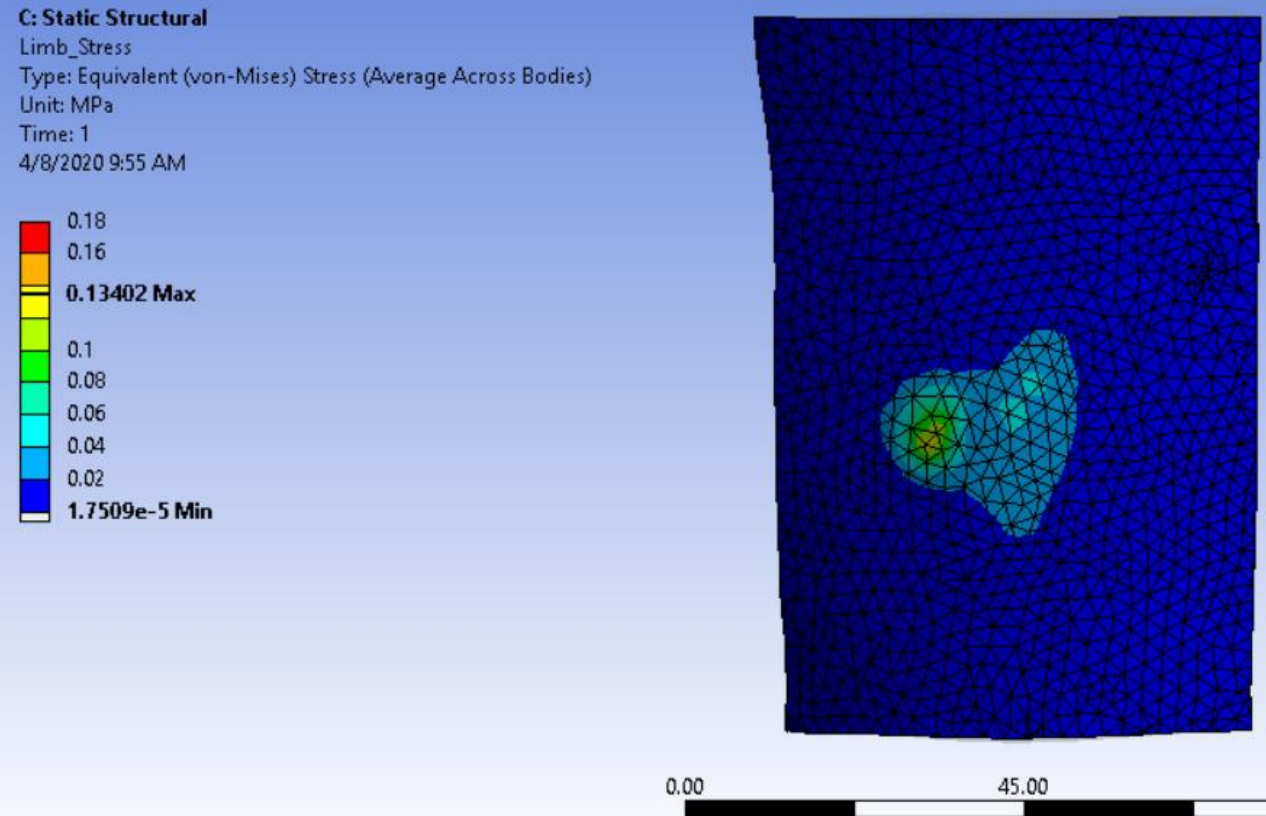

0.00 45.00

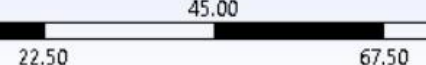
$90.00(\mathrm{~mm})$

Figure B1. Limb Surface Stress [MPa] with Silicone Liner at the Fibular Head 


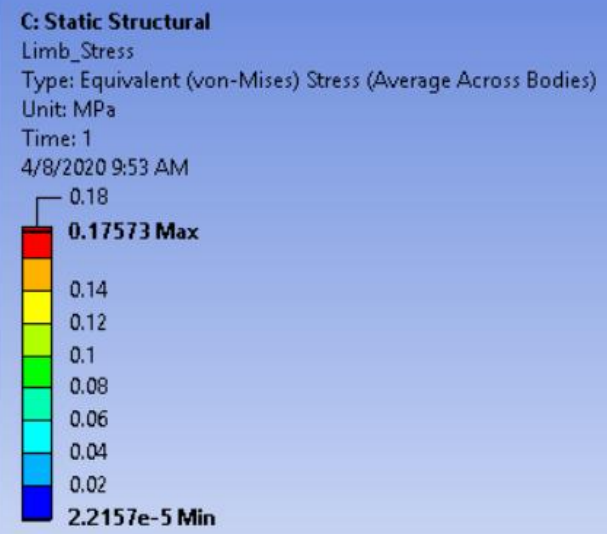

Type: Equivalent (von-Mises) Stress (Average Across Bodies)

Unit: $\mathrm{MPa}$

Time: 1

4/8/2020 9:53 AM

$\Gamma^{0.18} 0.17573 \mathrm{Max}$

0.14

0.12

0.1
0.08

0.06

0.04

0.02
$2.2157 \mathrm{e}-5 \mathrm{Min}$

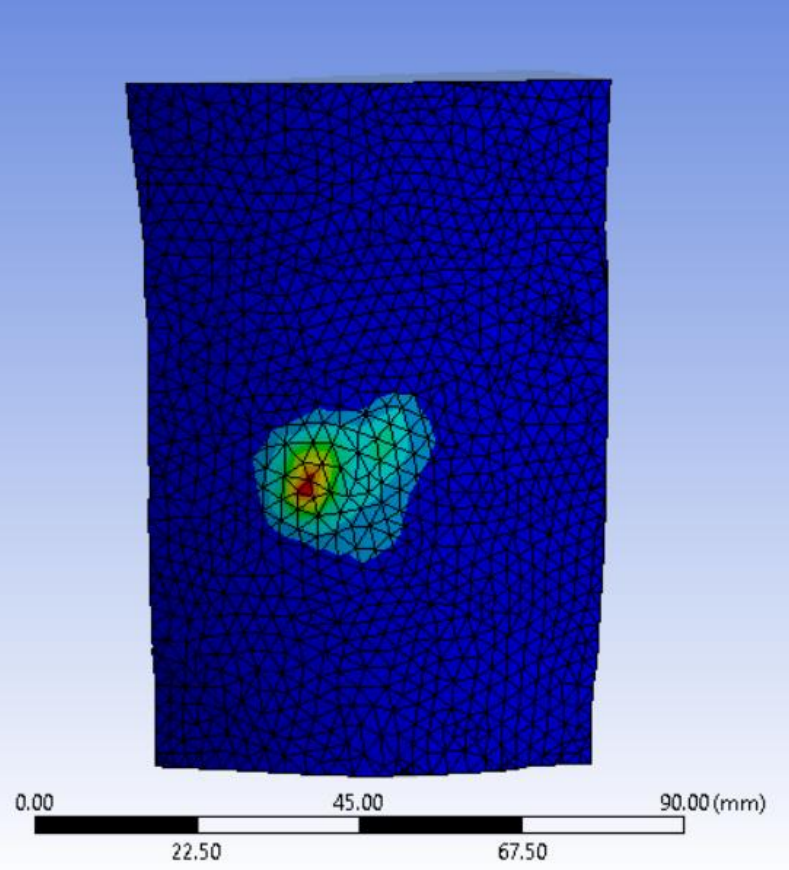

Figure B2. Limb Surface Stress [MPa] with Practitioner Prescribed Inlay at the Fibular Head

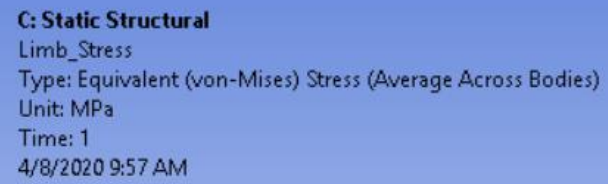

Type: Equivalent (von-Mises) Stress (Average Across Bodies)

Unit: $\mathrm{MPa}$

Time: 1

4/8/20209:57 AM

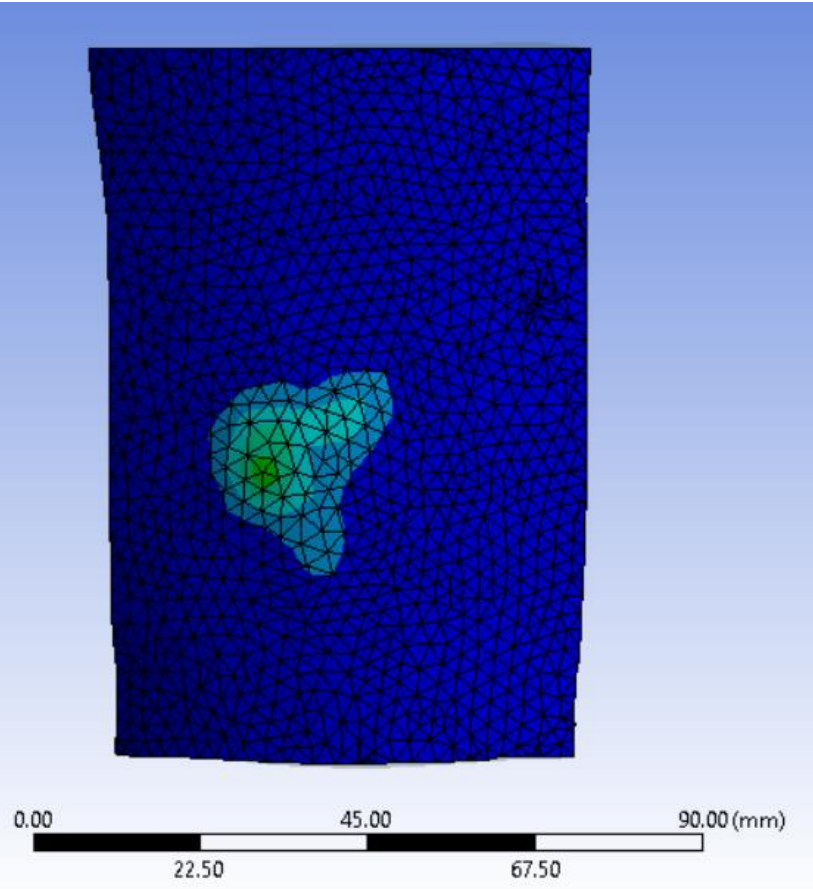

Figure B3. Limb Surface Stress [MPa] with Optimized Mat. Properties Inlay at the Fibular Head 
C: Static Structural

Limb_Stress

Type: Equivalent (von-Mises) Stress (Average Across Bodies)

Unit: $\mathrm{MPa}$

Time: 1

4/8/2020 9:59 AM
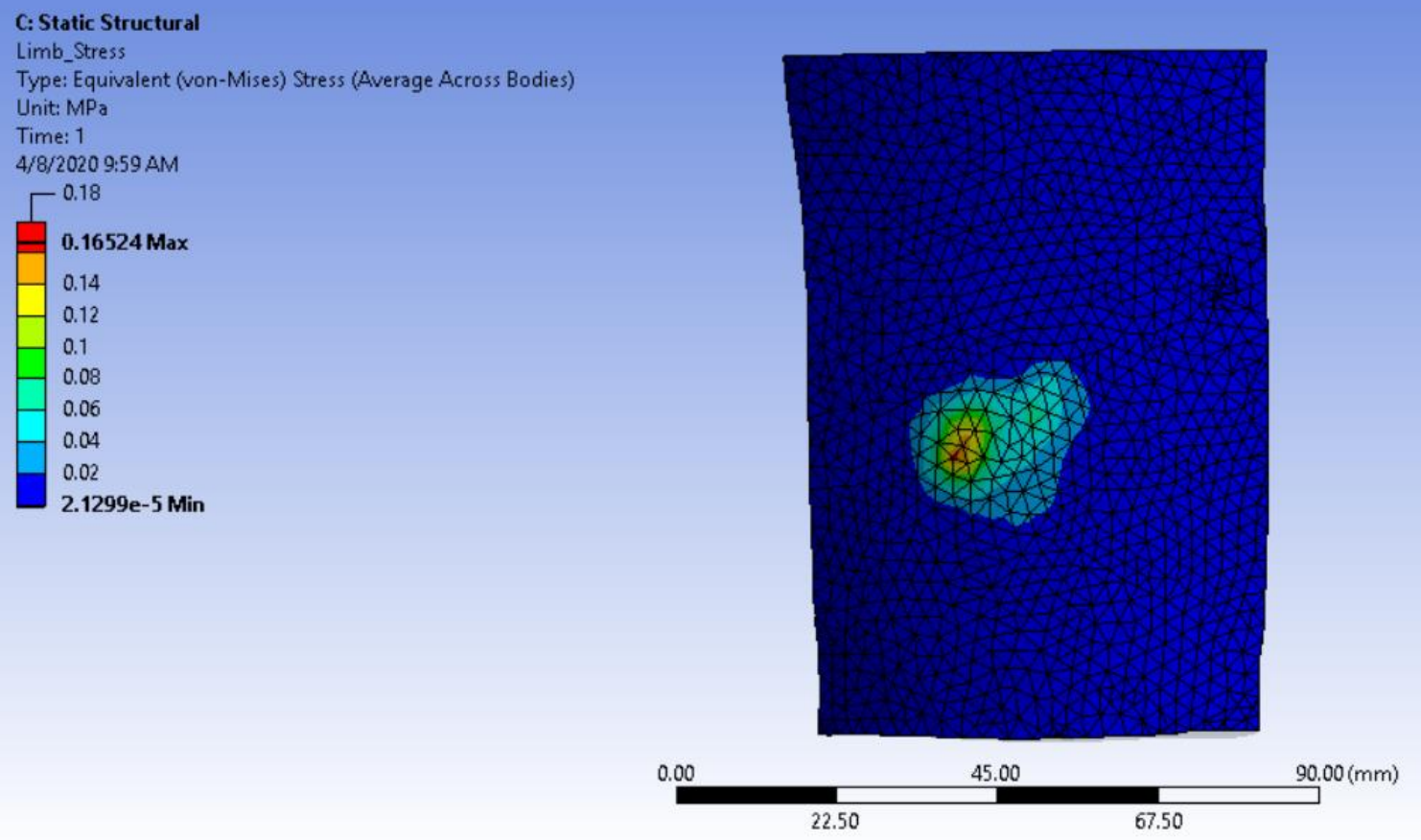

Figure B4. Limb Surface Stress [MPa] with Optimized Mat. Layout Inlay at the Fibular Head

C: Static Structural

Limb_Stress

Type: Equivalent (von-Mises) Stress (Average Across Bodies)

Unit: MPa

Time: 1

4/8/2020 10:01 AM
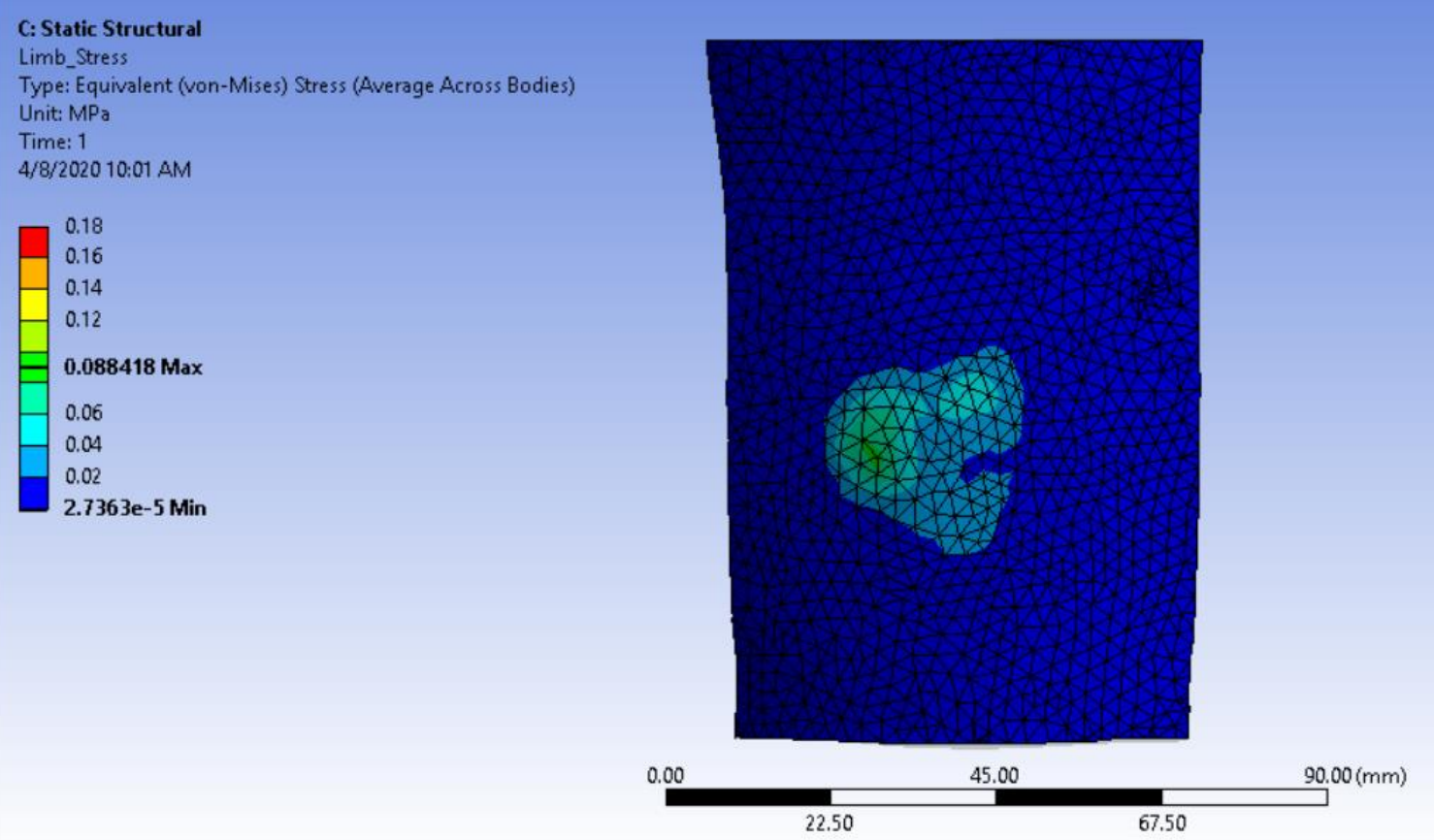

Figure B5. Limb Surface Stress [MPa] with Optimized Mat. Properties and Layout Inlay at the Fibular Head 

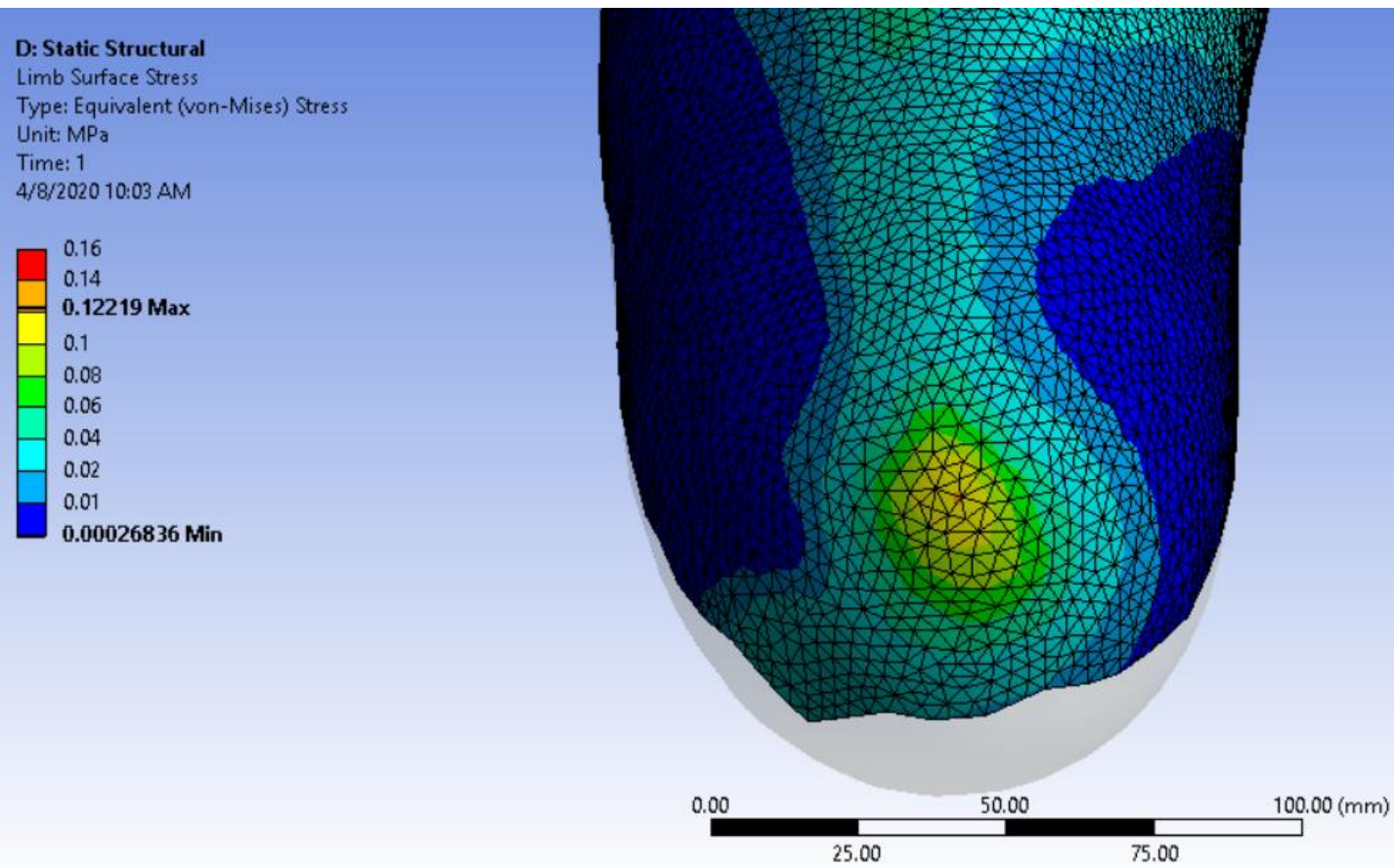

Figure B6. Limb Surface Stress [MPa] with Silicone Liner at the Tibial End

\section{D: Static Structural}

Limb Stress

Type: Equivalent (von-Mises) Stress

Unit: $\mathrm{MPa}$

Time: 1

4/8/2020 10:07 AM

\begin{tabular}{|l|l}
0.16 \\
0.14 \\
0.12 \\
0.1 \\
$0.079494 \mathrm{Max}$ \\
0.06 \\
0.04 \\
0.02 \\
0.01 \\
$0.00059728 \mathrm{Min}$
\end{tabular}
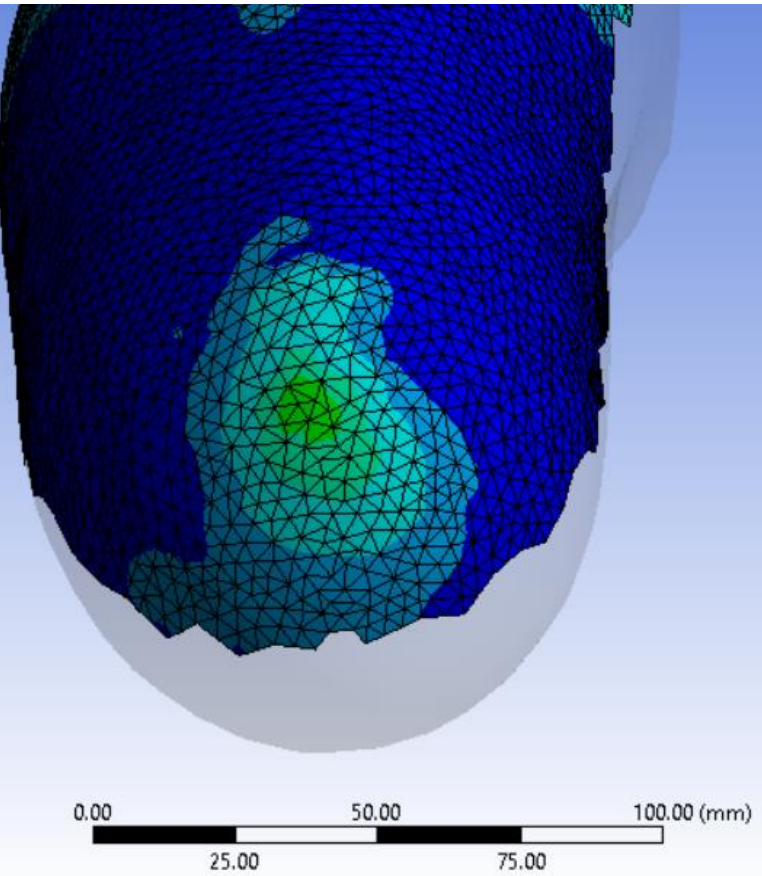

Figure B7. Limb Surface Stress [MPa] with Practitioner Prescribed Inlay at the Tibial End 
D: Static Structural

Limb Lower Stress

Type: Equivalent (von-Mises) Stress

Unit: MPa

Time: 1

4/8/2020 10:22 AM
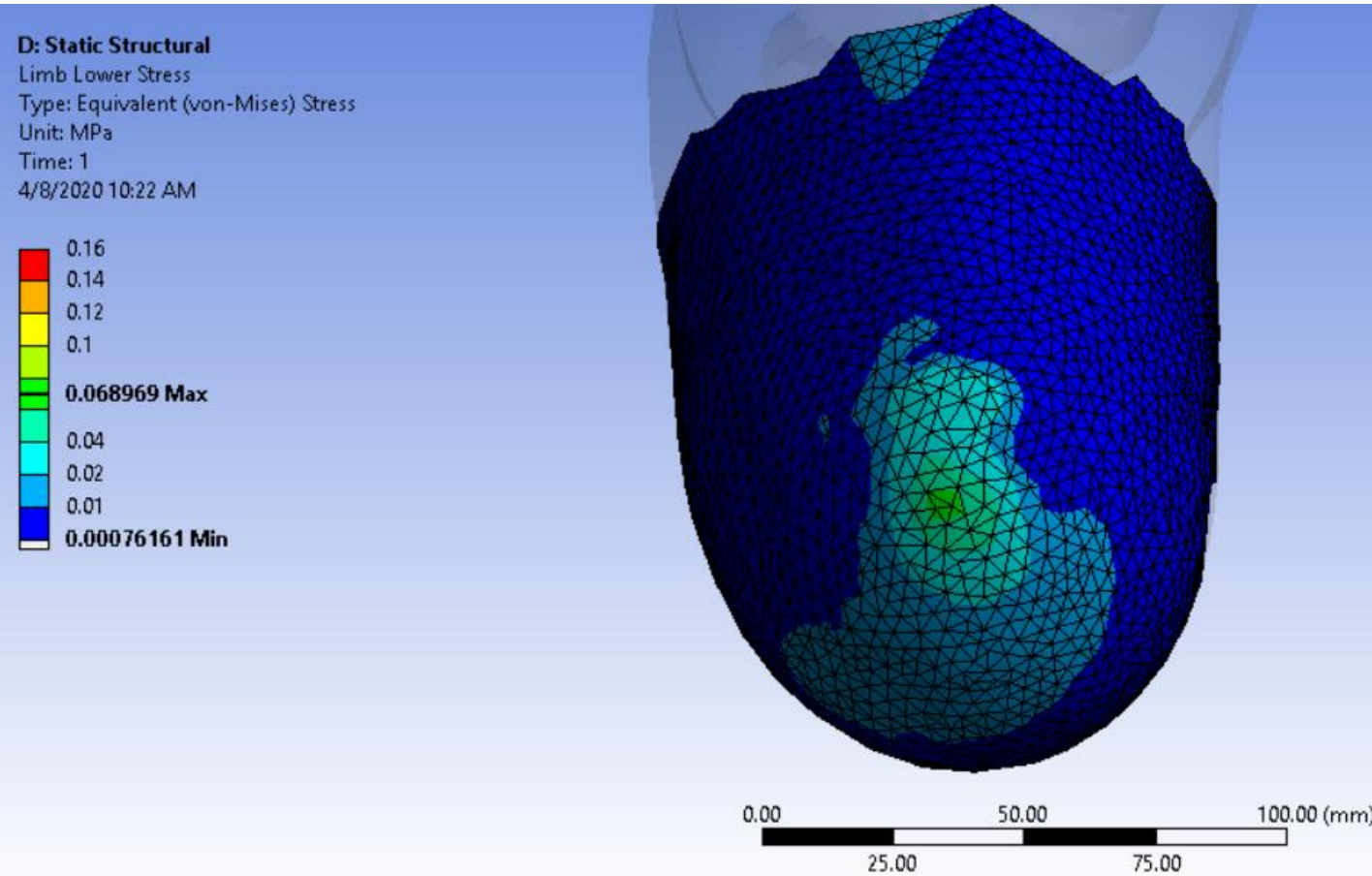

Figure B8. Limb Surface Stress [MPa] with Optimized Mat. Properties Inlay at the Tibial End
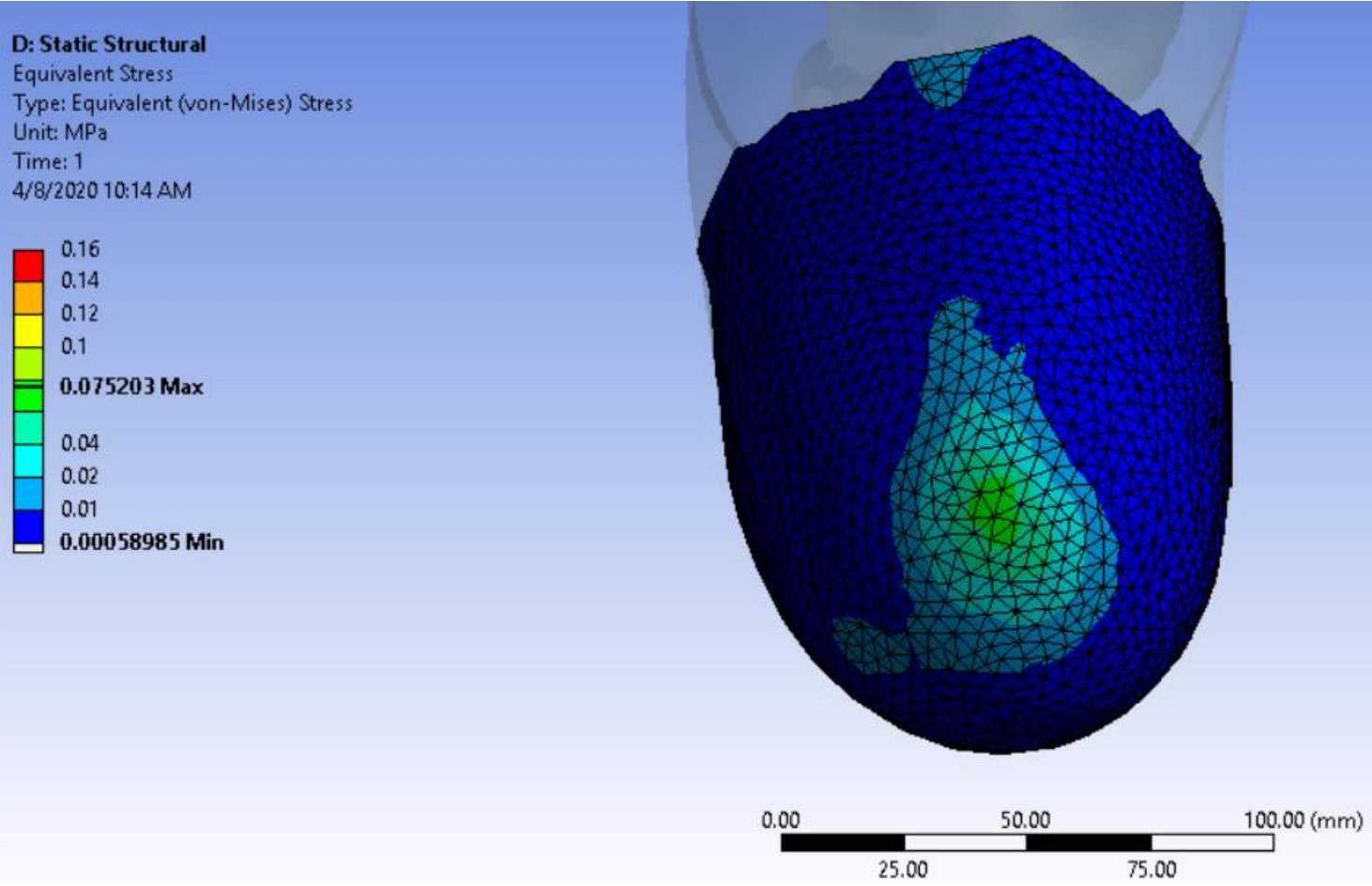

Figure B9. Limb Surface Stress [MPa] with Optimized Mat. Layout Inlay at the Tibial End 

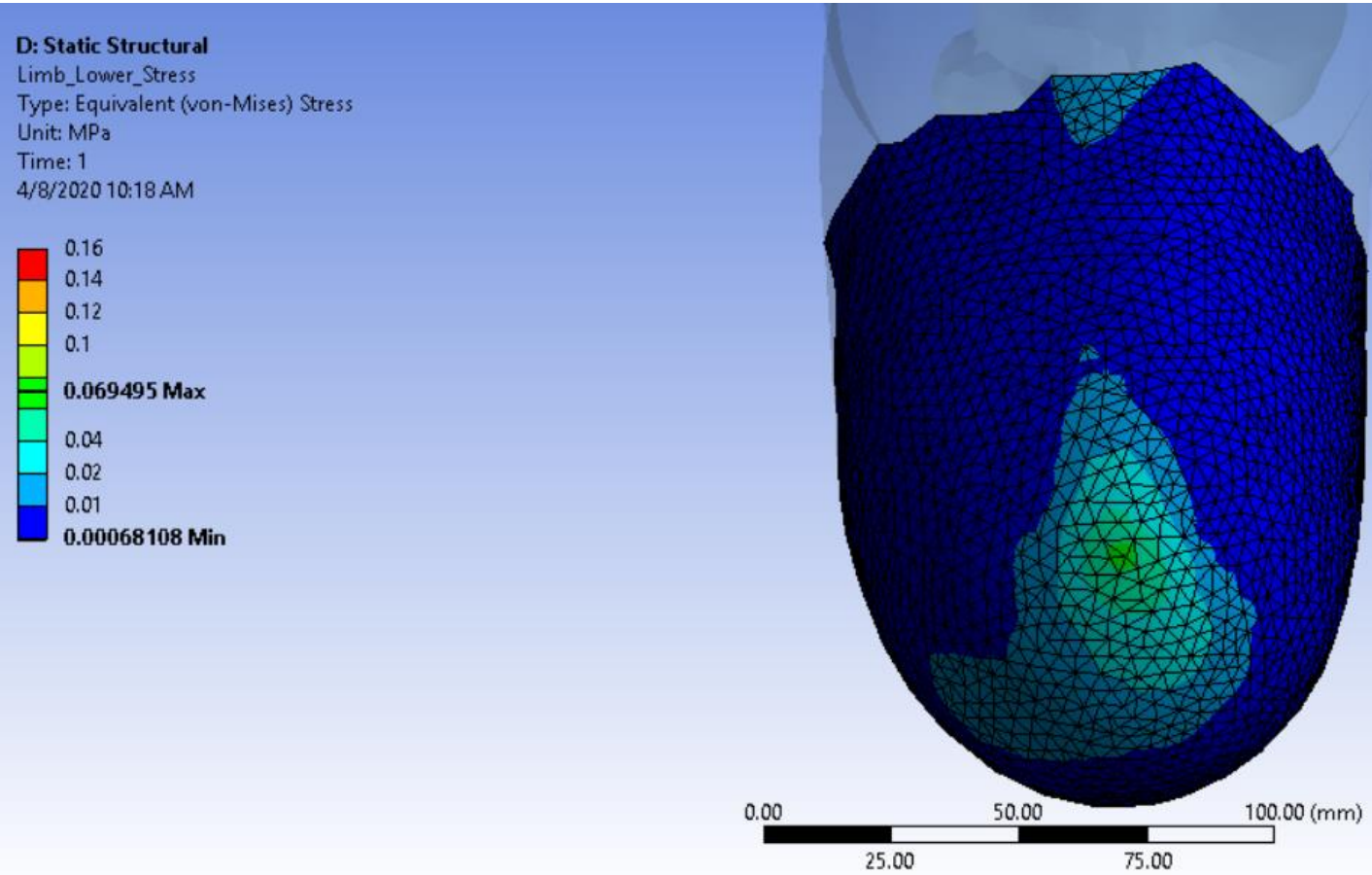

Figure B10. Limb Surface Stress [MPa] with Optimized Mat. Properties and Layout Inlay at the Tibial End

\section{D: Static Structural}

Limb Surface Stress

Type: Equivalent (von-Mises) Stress

Unit: MPa

Time: 1

4/8/2020 10:03 AM
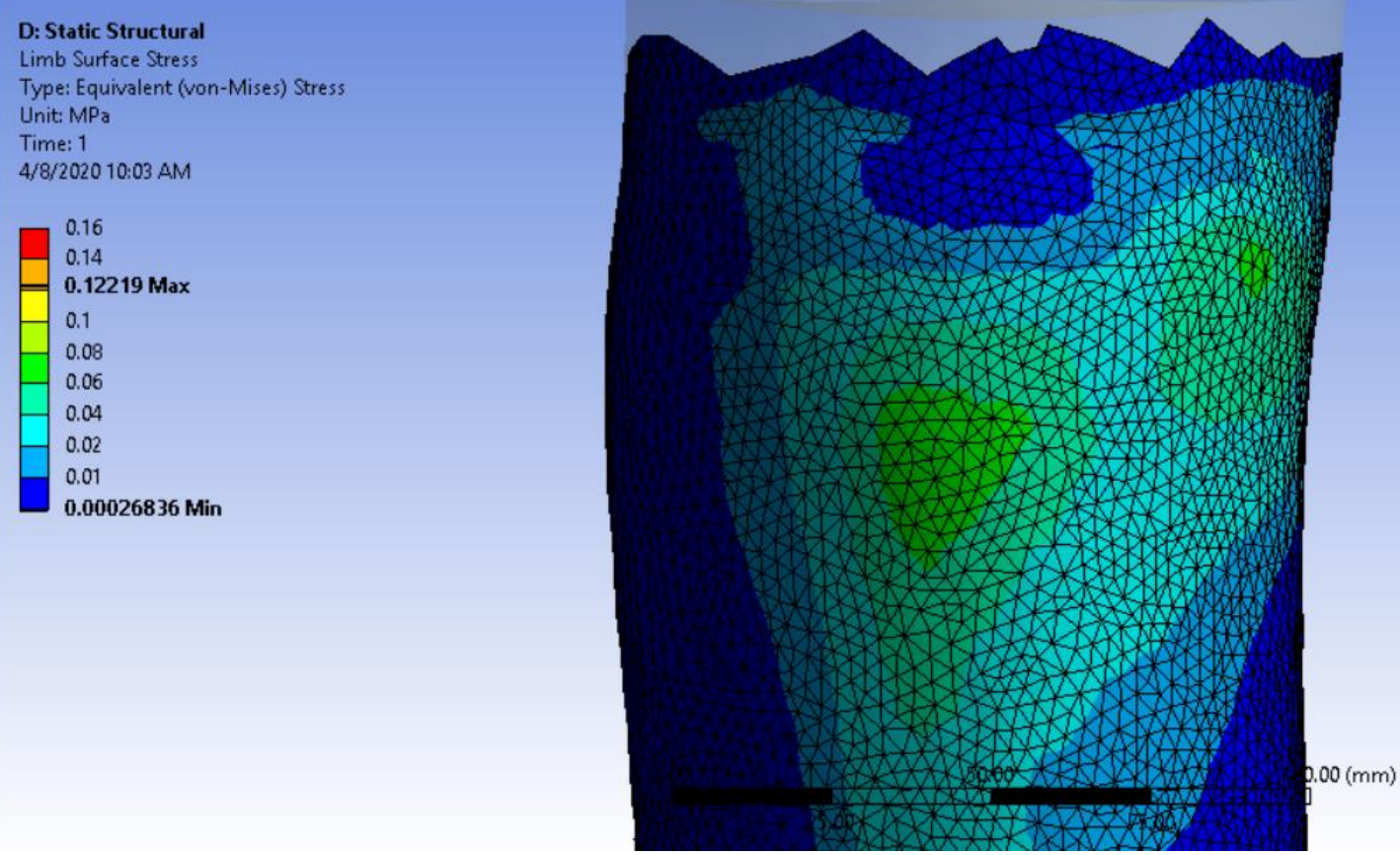

Figure B11. Limb Surface Stress [MPa] with Silicone Liner at the Patellar Tendon 
D: Static Structural

Limb Stress

Type: Equivalent (von-Mises) Stress

Unit: $\mathrm{MPa}$

Time: 1

4/8/2020 10:07 AM
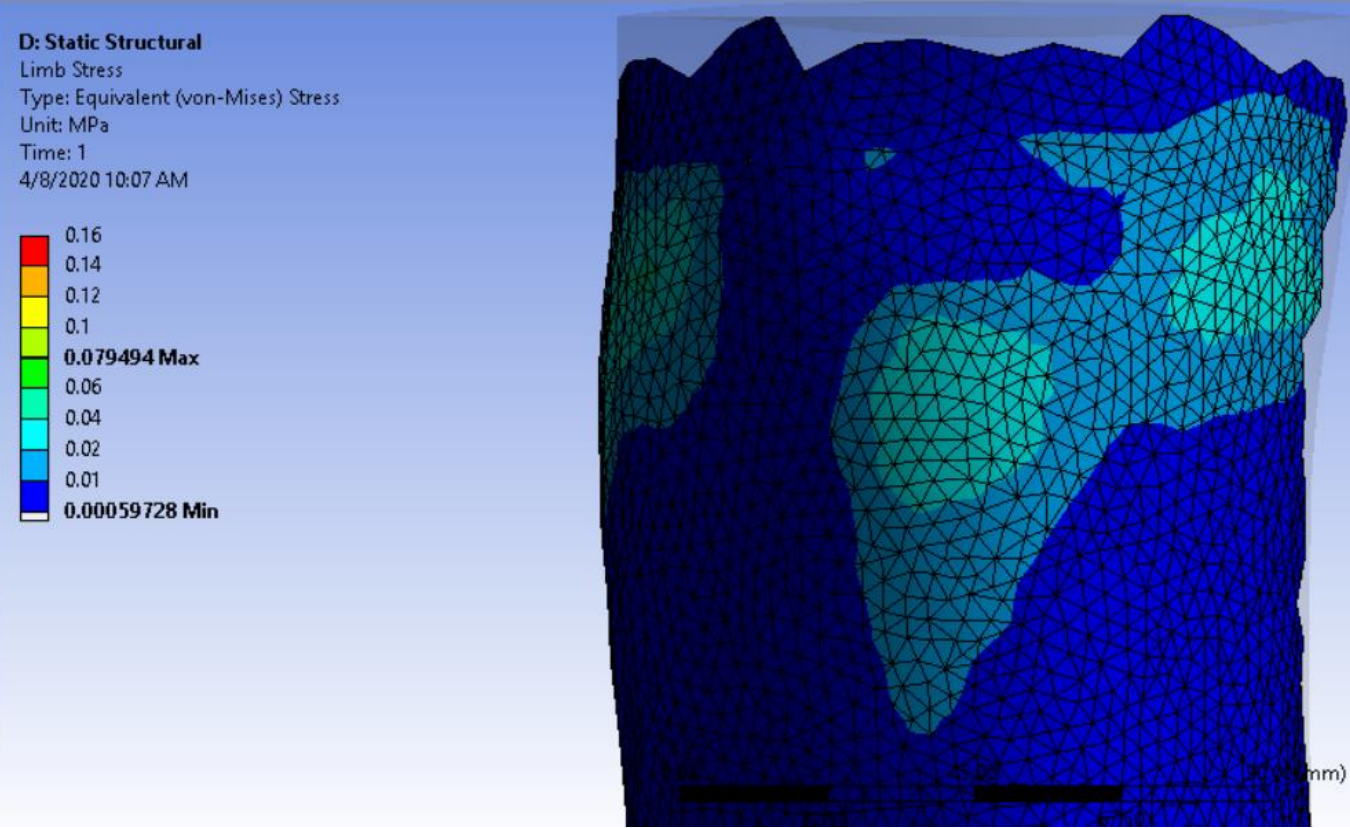

Figure B12. Limb Surface Stress [MPa] with Practitioner Prescribed Inlay at the Patellar Tendon

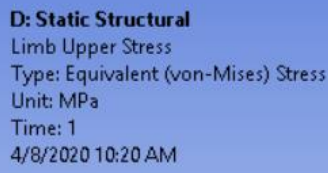

Type: Equivalent (von-Mises) Stress

Unit: MPa

Time: 1

4/8/202010:20 AM

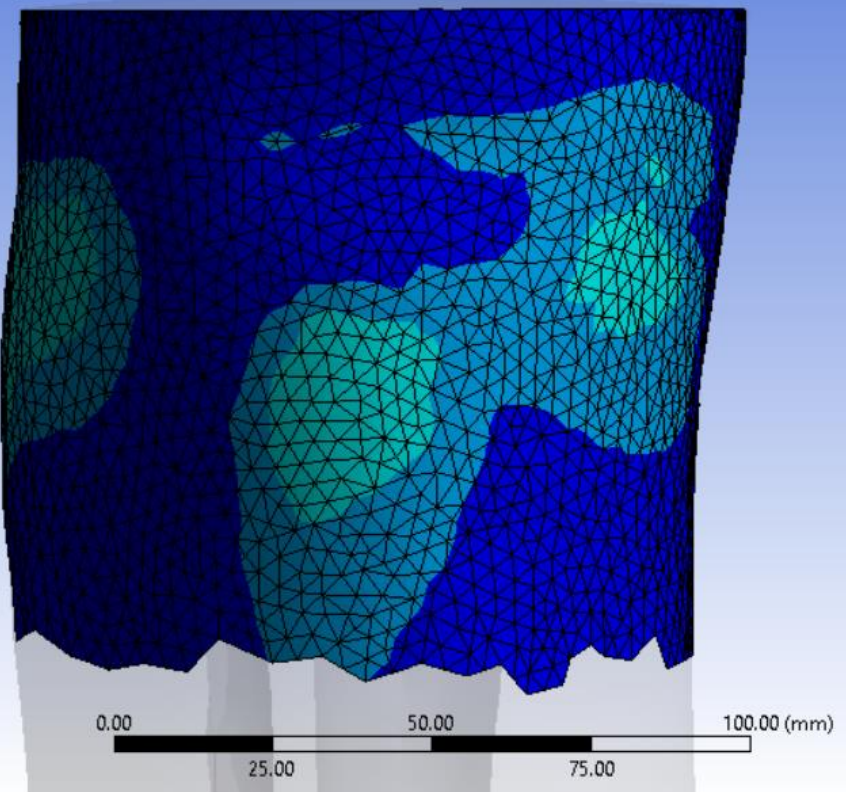

Figure B13. Limb Surface Stress [MPa] with Optimized Mat. Properties Inlay at the Patellar Tendon 


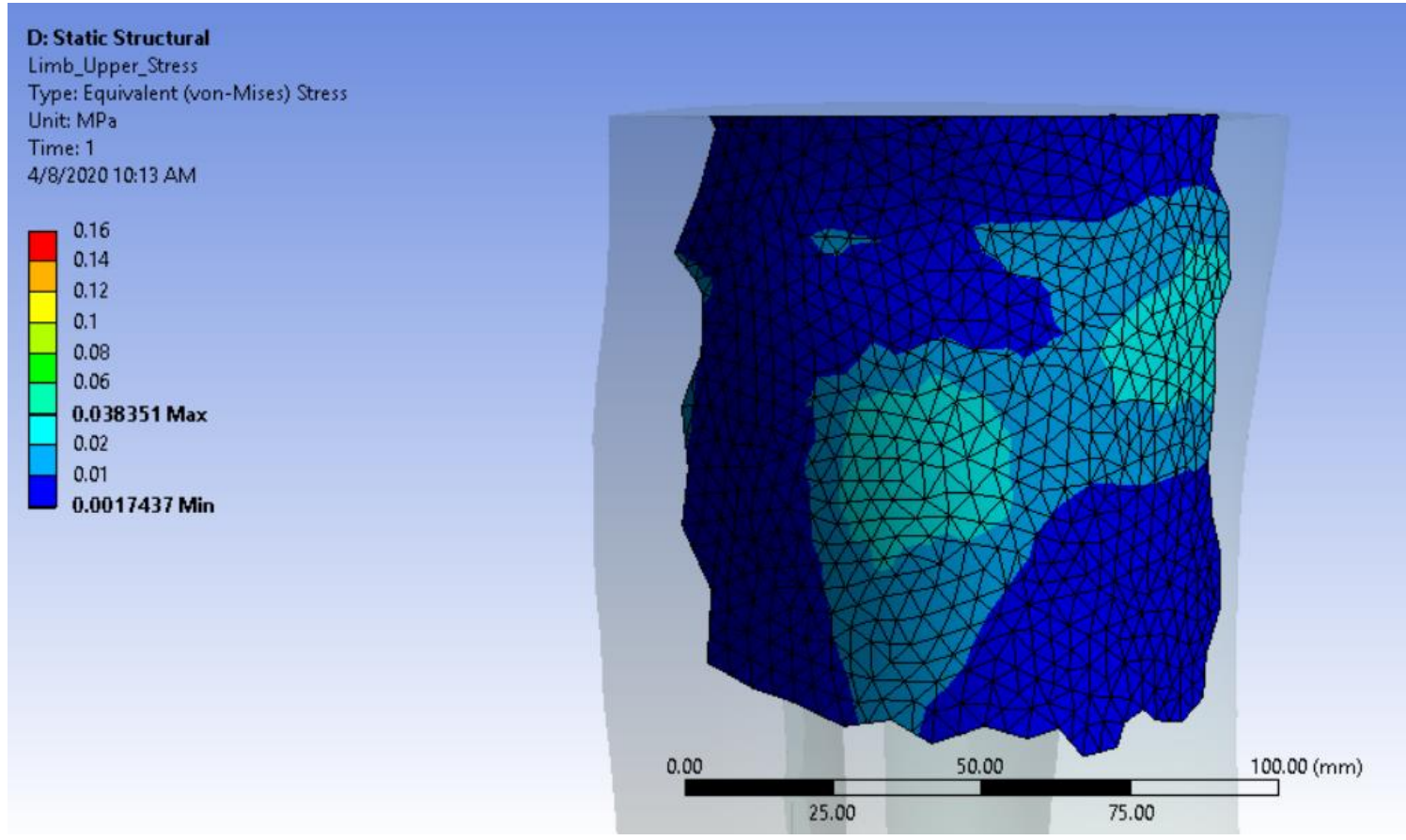

Figure B14. Limb Surface Stress [MPa] with Optimized Mat. Layout Inlay at the Patellar Tendon

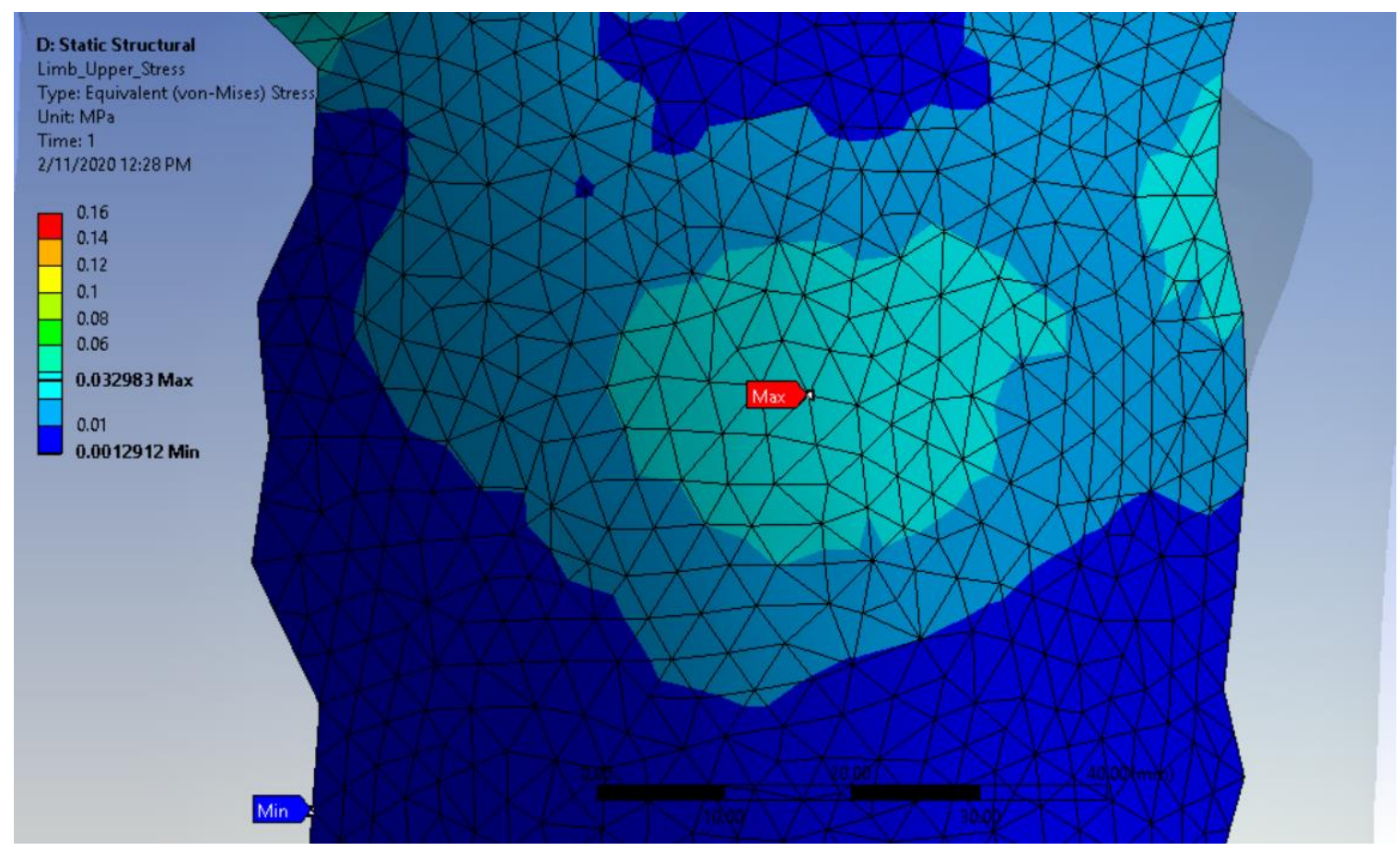

Figure B15. Limb Surface Stress [MPa] with Optimized Mat. Properties and Layout Inlay at the Patellar Tendon 


\section{REFERENCES}

[1] Gailey, R., \& Allen, K. (2008). Review of Secondary Physical Conditions Associated with Lower-limb Amputation and Long-term Prosthesis Use. Journal of Rehabilitation Research and Development, 45(1), 15-30.

[2] Raichle, K., Hanley, M., Kadel, N., Campbell, K., Phelps, E., \& Smith, D. (2008). Prosthesis Use in Persons with Lower and Upper-limb Amputation. Journal of Rehabiliation Research \& Development, 45(7), 961-972.

[3] Robbins, C. B., Vreeman, D. J., Sothmann, M. S., Wilson, S. L., \& Oldridge, N. B. (2009). A Review of the Long-Term Health Outcomes Associated With WarRelated Amputation. Military Medicine, 174(6), 588-592.

[4] Pirouzi, G., Abu Osman, N. A., Eshraghi, A., Ali, S., Gholizadeh, H., \& Wan Abas, W. (2014). Review of the Socket Design and Interface Pressure Measurement for Transtibial Prosthesis. The Scientific World Journal, 2014.

[5] Klute, G. K., Glaister, B. C., \& Berge, J. S. (2010). Prosthetic Liners for Lower Limb Amputees: A Review of the Literature. Prosthetics and Orthotics International, 34(2), 146-153.

[6] Hachisuka, K., Nakamura, T., Ohmine, S., Shitama, H., \& Shinkoda, K. (2001). Hygiene Problems of Residual Limb and Silicone Liners in Transtibial Amputees Wearing the Total Surface Bearing Socket. Archives of Physical Medicine and Rehabilitation, 82.

[7] Baars, E., \& Geertzen, J. (2005). Literature Review of the Possible Advantages of Silicon Liner Socket Use in Trans-tibial Prostheses. Prosthetics and Orthotics International, 29(1), 27-37.

[8] Zhang, M., \& Lee, W. C. (2006). Quantifying the Regional Load-Bearing Ability of Trans-Tibial Stumps . Prosthetics and Orthotics International, 30`(1), 25-34.

[9] Jia, X., Zhang, M., \& Lee, W. C. (2004). Load Transfer Mechanics Between Transtibial Prosthetic Socket and Residual Limb-dynamic Effects. Journal of Biomechanics, 37, 1371-1377. 
[10] Lenka , P. K., \& Choudhury, A. R. (2011). Analysis of Trans Tibial Prosthetic Socket Materials Using Finite Element Method . J. Biomedical Science and Engineering, 762-768.

[11] Lin, C.-C., Chang, C.-H., Wu, C.-L., Chung, K.-C., \& Liao, I.-C. (2004). Effects of Liner Stiffness for Trans-Tibial Prosthesis: A Finite Element Contact Model. Medical Engineering \& Physics , 26, 1-9.

[12] Boutwell, E., Stine, R., Hansen, A., Tucker, K., \& Gard, S. (2012). Effect of Prosthetic Gel Liner Thickness on Gait Biomechanics and Pressure Distribution within the Transtibial Socket . JRRD, 227-240.

[13] Armstrong, D., Peters, E., Athanastou, K., \& Lavery, L. (1998). Is there a critical level of plantar foot pressure to identify patients at risk for neuropathic foot ulcertation? J Foot Ankle Surgery, 303-307.

[14] Muller , M. J., Zou, D., \& Lott, D. J. (2005). "Pressure Gradient" as an Indicator of Planta Skin Injury. Diabetes Care, 2908-2912.

[15] Johnson, K. (1987). Contact Mechanics. Cambridge, UK: Cambridge University Press.

[16] Hanspal R. S., Fisher K. and Nieveen R. (2003). "Prosthetic Socket Fit Comfort Score," Disability and Rehabilitation, vol. 25, no. 22, pp. 1278-1280.

[17] Downic W., Leatham P., Rhind V., Wright V., Branco J. and Anderson J. (1978). "Studies with Pain Rating Scales," Annals of Rheumatic Diseases, vol. 37, pp. $378-381$.

[18] Legro M., Reiber G., Smith D., del Aguila M., Larsen J. and Boone D.. (1998). "Prosthesis Evaluation Questionaire for Persons with Lower Limb Amputations: Assessing Prosthesis-related Quality of Life," Archives of Physical Medicine and Rehabilitation, vol. 79, pp. 931-938.

[19] Lee W. C., Zhnag M. and Mak A. F.. (2005). "Regional Differences in Pain Threshold and Tolerance of the Transtibial Residual Limb: Including the Effects of Age and Interface Material," Arch Phys Med Rehabil, vol 85, no. 4, pp. 641649.

[20] Ebrahimzadeh M. and Hariri S. (2009). "Long-Term Outcomes of Unilateral Transtibial Amputations," Military Medicine, vol. 174, no. 6, pp. 593-597, 2009. 
[21] Flor H. (2002). "Phantom-Limb Pain: Characteristics, Causes, and Treatments," The Lancet Neurology, vol. 1, no. 3, pp. 182-189.

[22] Meulenbelt HE, Geertzen JH, Dijkstra PU, Jonkman MF. (2007). Skin Problems in Lower Limb Amputees: An Overview by Case Reports. J Eur Acad Dermatol Venereol; 21(2):147-155

[23] Levy SW. (1995). Amputees: Skin Problems and Prostheses. Cutit;55(5):297-301

[24] Highsmith JT, Highsmith MJ. (2007). Common Skin pathology in LE prosthesis users. JAAPA;20(11):33-36,47.

[25] Sanders , J., Daly, C., Cummings, W., Reed, R., \& Marks, R. (1994). A Measurement Device to Assist Amputee Prosthetic Fitting . Journal of Clinical Engineering , 6371.

[26] Sanders, J. E., Greve, J. M., Mitchell, S. B., Zachariah, \& Santosh, G. (1998). Material Properties of Commonly-Used Interface Materials and Their Static Coefficients of Friction with Skin and Socks. Journal of Rehabilitation Research and Development, 35, 161-176.

[27] Kristinsson, O. (1993). The ICEROSS concept: A Discussion of a Philosophy. Prosthetics and Orthotics International, 17, 49-55.

[28] Emrich R, Slater K. (1978).vComparative Analysis of Below-Knee Prosthesis. Prosthet Orthot Int; 2(1):3-7.

[29] Sanders JE, Nicholson BS, Zachariah SG, Cassisi DV, Karchin A, Fergason JR. (2004). Testing of Elastomeric Liners Used in Limb Prosthetics: Classification of 15 Products By Mechanical Performance. J Rehabil Res Dev; 41(2):175-275.

[30] Dillingham TR, Pezzin LE, MacKenzie EJ, Burgess AR. (2001). Use and Satisfaction with Prosthetic Devices Among Persons with Trauma-Related Amputations: A Long-Term Outcome Study. AM J Phys Med Rehabil; 80(8):563571.

[31] Legro MW, Reiber G, del Aguila M, Ajax MJ, Boone DA, Larsen JA. (1999). Issues of Importance Reported By Persons with Lower Limb Amputations and Prostheses. J Rehabil Res Dev;36(3):155-163.

[32] Hagberg K, Branemark R. (2001). Consequences of Non-Vascular Trans-Femoral Amputation: A Survey of Quality of Life, Prosthetic Use and Problems. Prosthet Orthot Int.;25(3):186-194. 
[33]Hachisuka K, Dozono K, Ogata H, Ohmine S, Shitama H, Shinkoda K. (1998). Total Surface Bearing Below-Knee Prosthesis: Advantages, Disadvantages, and Clinical Implications. Arch Phys Med Rehabil.; 79(7): 783-389.

[34] Hachisuka K, Nakamura T, Ohmine S, Shitame H, Shinkoda K. (2001). Hygiene Problems of Residual Limb and Silicone Liners in Transtibial Amputees Wearing the Total Surface Bearing Socket. Arch Phys Med Rehabil; 82(9): 1286-1290.

[35] Hachisuka K, Matsushima Y, Ohmine S, Shitama H, Shinkoda K. (2001). Moisture Permeability of the Total Surface Bearing Prosthetic Socket With a Silicone Liner: Is it Superior To The Patella-Tendon Bearing Prosthetic Socket? J Uoeh;23(3): 225-232.

[36] Klute GK, Rowe GI, Mamishec AV, Ledoux WR. (2007). The Thermal Conductivity of Prosthetic Sockets And Liners. Prosthet Orthot Int; 31(3):292-299.

[37] Hafner, B. J., Cagle, J., Allyn, K. J., \& Sanders, J. E. (2017). Elastomeric Liners forPeople with Transtibial Amputation: Survey of Prosthetists' Clincal Practices . Prosthetics and Orthotics International, 149-156.

[38] Pendry, J. B. (2000). Negative Refraction Makes a Perfect Lens. Physical Review Letters, 3966-3969.

[39] Shelby, R. A., Smith, D. R., \& Schultz, S. (2001). Experimental Verification of a Negative Index of Refraction. Science, 77-79.

[40] Smith, D. R., Pendry, J. B., \& Wiltshire, M. C. (2004). Metamaterials and Negative Refraction Index. Science, 788-792.

[41] Cai, W., \& Shalaev, V. (2010). Optical Metamaterials: Fundamentals and Applications. New York: Springer.

[42] Marques, R., Martin, F., \& Sorolla, M. (2011). Metamaterials with negative parameters: Theory, design and microwave Applications. New Jersey: John Wiley \& Sons.

[43] Pendry JB. (2000). Negative Refraction Makes a Perfect Lens. Phys Rev Lett;85:3966.

[44] Shelby RA, Smith DR, Schultz S. (2001). Experimental Verification of a Negative Index of Refraction. Science;292:77-9.

[45] Smith DR, Pendry JB, Wiltshire MC. (2004). Metamaterials and Negative Refractive Index. Science;305:788-92. 
[46] Yu, X., Zhou, J., Liang, H., Jiang, Z., \& Wu, L. (2018). Mechanical Metamaterials Associated with Stiffness, Rigidity and Compressibility: A brief review. Progress in Materials Science, 114-173.

[47] Mullin T, Deschanel S, Bertoldi K, Boyce M. (2007). Pattern Transformation Triggered by Deformation. Phys Rev Lett 2007;99:084301.

[48] Overvelde JTB, Shan S, Bertoldi K. (2012). Compaction Through Buckling In 2D Periodic, Soft and Porous Structures: Effect of Pore Shape. Adv Mater;24:2337-42.

[49] Yang, H., Ganzosch, G., Giorgio, I. (2018). Material Characterization and Computations of a Polymeric Metamaterial With a Pantographic Substructure. Z. Angew. Math. Phys. 69, 105

[50] Bodaghi M, Damanpack A.R., Hu G.F., Liao W.H. (2017). Large Deformations of Soft Metamaterials Fabricated By 3D Printing. Materials and Design. 131, 81:91.

[51] Cagle, J. C., Reinhall, P. G., Hafner , B. J., \& Sanders, J. E. (2017). Development of Standardized Material Testing Protocols for Prosthetic Liners. Journal of Biomechanical Engineering.

[52] Lee, W. C., Zhang, M., Jia, X., \& CHeung, J. T. (2004). Finite Element Modeling of the Contact Interface Between Trans-Tibial Residual Limb and Prosthetic Socket. Medical Engineering \& Physics.

[53] Cagle, J. C., Reinhall, P. G., Allyn, K. J., McLean, J., Hinrichs, P., Hafner, B. J., \& Sanders, J. E. (2018). A Finite Element Model to Assess Transtibial Prosthetic Sockets with Elastomeric Liners . Medical \& Biological Engineering \& Computing , 1227-1240.

[54] Zhang, M., Lord, M., Turner-Smoth, A. R., \& Roberts, V. C. (1995). Development of a Non-linear Finite Element Modelling of the Below-knee prosthetic socket interface . Medical Engineering \& Physics, 559-566.

[55] Steer, J. W., Worsley, P. R., Browne, M., \& Dickinson, A. S. (2019). Predictive Prosthetic Socket Design: Part 1- Population-Based Evaluation of Transtibial Prosthetic Sockets by FEA-Driven Surrogate Modelling . Biomechanics and Modeling in Mechanbiology.

[56] Dickinson, A. S., Steer, J. W., \& Worsley, P. R. (2017). Finite Element Analysis of the Amputated Lower Limb: A Systematic Review and Recommendations. Medical Engineering \& Physics, 43(5), 1-18. 
[57] Silver-Thorn, M. B., \& Childress, D. S. (1995). Parametric Analysis Using the Finite Element Method to Investigate Prosthetic Interface Stresses for Persons with Transtibial Amputation. Journal of Rehabilitation Research and Development, 227-238.

[58] DesJardins J., Stanley S. E., Przestrelski B., Pruett T.C., Hoeffner S.L., Kaluf B. D., "Variable Hardness Orthotic", Patent 10244818 B2, Publication 2019-04-02

[59] Przestrselski, B. T. (2017). In-Shoe Innovation: 3-D Printed Foot Orthotses. Clemson: Clemson University.

[60] O.H. Yeoh. (1993). Some Forms of the Strain Energy Function For Rubber. Rubber Chem. Technol., 66:745-771.

[61] Hauseux, P., Hale, JS., Cotin, S., Bordas, S. PA. (2018). Quantifying the Uncertainty in a Hyperelastic Soft Tissue Model with Stochastic Parameters. Applied Mathematical Modelling. 62:86-102.

[62] Thompson, JS., Walton, S., Hassan, O., Rolland, SA., Sienz, J. (2017). The Use of CFD and Multi-Objective Optimization Techniques to Customize an Industrial Pre-Mizer. Struct Multidisc Optim. 55:2339-2351.

[63] Satterfield Z, Kulkarni N, Fadel G, Li G, Coutris N, Castanier MP. Unit Cell Synthesis for Design of Materials with Targeted Nonlinear Deformation Response ASME. J. Mech. Des. 2018; 139(8)

[64] Yeung, L.F., Leung, A.K., Zhang, M., \& Lee, W.C. (2013). Effects of Long-Distance Walking on Socket-Limb Interface Pressure, Tactile Sensitivity, and Subjective Perceptions of Trans-Tibial Amputees. Disability and Rehabilitation, 888-893.

[65] Congdon, Wes. (2011) Standard of Care: Lower Extremity Amputation. Brigham and Women's Hospital: Department of Rehabilitation Services, 1-46. 